Center for

Mathematical Economics

Working Papers

August 2018

\title{
Dynamic Consistency in Incomplete Information Games with Multiple Priors
}

Marieke Pahlke

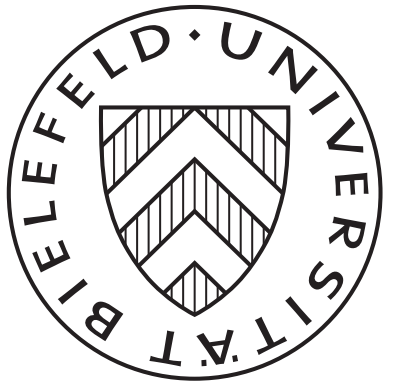




\title{
Dynamic Consistency in Incomplete Information Games with Multiple Priors*
}

\author{
Marieke Pahlke
}

August 17, 2018

\begin{abstract}
This paper generalizes the concept of Sequential Equilibrium to allow for ambiguous incomplete information about types or states. We characterize conditions that ensure existence of Sequential Equilibria under ambiguous incomplete information. Under these conditions players form subjective prior belief sets that satisfy a rectangularity condition which leads to dynamically consistent behavior. Furthermore, we give an example which shows that ambiguity can introduce new Sequential Equilibria.

Key words and phrases: sequential equilibrium, ambiguity, dynamic consistency, multiple priors, imprecise information

JEL subject classification: C73, D81
\end{abstract}

\section{Introduction}

Starting with Ellsberg (1961) it has been shown that agents behave differently if they face ambiguity instead of risk i.e. situations where the probability distributions of outcomes is not known. Since then, ambiguity has been introduced into many decision and game theoretic models and has been supported by experimental and empirical evidence. ${ }^{1}$ Furthermore, ambiguity has been used in many applications e.g. in mechanism design (e.g Bose, Ozdenoren, and Pape (2006)) and asset pricing (e.g. Ju and Miao (2012)). However, it has rarely been used in dynamic games. ${ }^{2}$

The formation of beliefs plays an important role in games with incomplete information and motivated the definition of different equilibrium concepts as e.g. Bayesian Perfect Nash

${ }^{*}$ I would like to thank Frank Riedel and Peter Klibanoff for their comments and suggestions. Financial support from BiGSEM, BGTS and DAAD is gratefully acknowledged.

${ }^{\dagger}$ Bielefeld University, Center for Mathematical Economics, 33615 Bielefeld, Germany marieke.pahlke@uni-bielefeld.de

${ }^{1}$ See Section 6 in Etner, Jeleva, and Tallon (2012) for a recent review of experimental literature with ambiguity.

${ }^{2} \mathrm{~A}$ few notable exceptions are discussed in Section 1.1. 
Equilibrium or Sequential Equilibrium. One essential assumption for these equilibrium concepts are rational players that use (whenever possible) Bayes' rule to update their beliefs after observing new information. In incomplete information games with ambiguity players are faced with a set of ex-ante beliefs instead of one belief. Therefore, before generalizing the concept of Sequential Equilibrium to games with incomplete ambiguous information we have to specify how players update their set of ex-ante beliefs. Different updating rules are defined in the literature but almost all of them can lead to dynamically inconsistent behavior in combination with maxmin expected preferences as introduced by Gilboa and Schmeidler (1989). Roughly speaking new information can lead to a change in the worst case belief which induces a change in the optimal strategy and therefore dynamically inconsistent behavior. This makes it impossible to use the standard concepts of Sequential and Bayesian Perfect Nash Equilibria and complicates the analysis of dynamic games with ambiguity.

To our knowledge this is the first paper that characterizes conditions that ensure dynamically consistent behavior in games with incomplete information and multiple priors. Using our setting in applications as e.g. mechanism design would allow to explore optimal dynamically consistent decisions or strategies in dynamic games with ambiguous incomplete information. Hence, the effect of ambiguity aversion and dynamically inconsistent behavior could be analyzed separately.

We assume prior by prior Bayesian updating, i.e. players update their set of beliefs by updating each belief in the ex ante belief set using Bayes' rule. We characterize conditions on the belief sets that lead to dynamically consistent behavior and therefore ensure the existence of Sequential Equilibria. These conditions are closely related to rectangularity as used in decision theoretic settings (e.g. Epstein and Schneider (2003)). ${ }^{3}$ Intuitively rectangularity captures the idea that players take their future worst case belief into account, when constructing their prior belief set. To be more specific, in our model ambiguity is represented by imprecise probabilistic information i.e., a common set of probability distributions over states or types. New information arises when a player observes an action played by his opponent. Observing an action played by an opponent does not only reveal information about his type but also about the strategy played by the opponent. Therefore, future information can be heterogeneous across players. We assume that players use their knowledge about the structure of the game (action sets, information sets, etc.) to anticipate which information they could get in the future. We will show that considering this knowledge and given the heterogeneous information structure and the common imprecise probabilistic information each player construct a prior belief set. These prior belief sets are heterogeneous across agents and satisfy a rectangularity condition for each agent.

Ellis (2018) and Aryal and Stauber (2014) argue that in games with multiple prior a common prior belief set can only be rectangular if there is no ambiguity. But they do not consider the differences in information structure of games and decision theoretic settings

\footnotetext{
${ }^{3}$ Rectangularity or sometimes called stability under pasting is a condition used in decision theory to ensure dynamic consistency in multiple prior settings. However, there are important differences between the information structure in games and decision theory.
} 
that lead to heterogeneous information and therefore heterogeneous beliefs sets, described above. We will show, that in our setting, despite the common imprecise probabilistic information, rectangular prior beliefs sets will differ across the players because of their different future information. Therefore, the critique of Ellis (2018) and Aryal and Stauber (2014) does not apply to our setting.

Furthermore, we show that due to rectangularity the interim worst case belief is the Bayesian update of the ex-ante worst case belief. Therefore, a completely mixed ex-ante equilibrium with rectangular beliefs implies an interim equilibrium with rectangular beliefs. As in games without ambiguity, deviations from an ex-ante equilibrium only occur in outof-equilibrium information sets. This relation between ex-ante and interim equilibria and beliefs illustrates dynamically consistent behavior under rectangular belief sets.

Finally we define Sequential Equilibria with rectangular beliefs analogously to Kreps and Wilson (1982). We prove existence and provide an example which shows that ambiguity induces new Sequential Equilibria that cannot occur without ambiguity.

The structure of this paper is as follows. In Section 1.1 we summarize related literature. In Section 2 we formulate the extensive-form game with ambiguity and define belief sets which satisfy rectangularity. Section 3 shows the existence and the relation of ex-ante and interim equilibria. In Section 4 we prove existence of Sequential Equilibria with rectangular beliefs. Furthermore, we give an example which shows that ambiguity might induce new Sequential Equilibria. Finally, Section 5 concludes.

\section{$1.1 \quad$ Literature}

There is a huge literature on ambiguity in games and decision theory. ${ }^{4}$ But only few work has been done on ambiguity in general dynamic games. ${ }^{5}$

Lo (1999) explores extensive form games with MEU. Instead of imposing conditions to ensure dynamic consistency he introduces an equilibrium concept which explicitly requires each player to know that strategies chosen in equilibrium are interim optimal. Kajii and Ui (2005) also formulate an incomplete information game with multiple prior. In their setting new information arises due to signals which are independent of the strategies. Therefore, the information setting is very close to decision theoretic settings and does not capture the strategic aspects that e.g. occur in signaling games. Liu and Xiong (2016) formulate a similar model as Kajii and Ui (2005) and show that rectangularity leads to dynamically consistent behavior. But as already mentioned the information structure is very similar to decision theory. In our model we extend the information structure such that information arises due to observed actions played by the opponents.

\footnotetext{
${ }^{4}$ For recent surveys of literature on ambiguity and axiomatic foundation see Gilboa (2009) and Gilboa and Marinacci (2016).

${ }^{5}$ There are some papers considering ambiguity in mechanism design, e.g. Bose and Renou (2014) and Tillio, Kos, and Messner (2016). Both show how ambiguity aversion can be exploited in mechanism design. Furthermore, Bose and Daripa (2009) use a dynamic auction mechanism and exploit dynamically inconsistent behavior.
} 
Muraviev, Riedel, and Sass (2017) explore extensive form games where players can use Ellsberg strategies, introduced by Riedel and Sass (2014). Ellsberg strategies extend mixed strategies to ambiguous strategies, i.e. instead of playing a probability distribution over the pure strategies a player chooses a set of probability distributions. They show that a rectangularity condition ensures outcome-equivalence between mixed and behavioral strategies but they do not formulate a general equilibrium concept for such games. However, similar to our paper, Muraviev, Riedel, and Sass (2017) have to construct a sequence of filtration to define rectangularity. The difference between our model and the model of Muraviev, Riedel, and Sass (2017) is the source of ambiguity. In Ellsberg games ambiguity arise due to ambiguous strategies. In our setting ambiguity arise due to incomplete ambiguous information about states or types.

The paper which is closest to our work is Hanany, Klibanoff, and Mukerji (2018). They also explore a finite extensive form multi-stage game with incomplete information, but use smooth ambiguity aversion instead of multiple piors. They show that updating beliefs with the so called smooth rule leads to dynamically consistent behavior which implies existence of Sequential Equilibria. This result is strongly correlated with the fact that smooth rule updating ensures dynamically consistent behavior in decision theoretic settings as shown by Hanany and Klibanoff (2009).

Sarin and Wakker (1998) and Epstein and Schneider (2003) define rectangularity for different decision theoretic settings. ${ }^{6}$ They show that rectangularity implies dynamically consistent behavior in their setting. Furthermore, Riedel, Tallon, and Vergopoulos (2017) explore a dynamic decision theoretic setting where given imprecise probabilistic information an agent chooses an act and a prior belief set. Roughly speaking, they show that an ambiguity averse agent which behaves dynamically consistently chooses a rectangular subjective prior set and evaluates acts according to the worst case belief given his subjective prior belief set.

Another way to rule out dynamically inconsistent behavior of MEU are updating rules proposed by Hanany and Klibanoff (2007). These rules only update a subset of the prior belief set using Bayes' rule, where roughly speaking the subset depends on the optimal ex ante choice. However, they show that some of their ambiguity-maximizing updating rules coincide with fully Bayesian updating, if the prior belief set is rectangular. We discuss the differences between these updating rules and our approach in Section 5.

\section{Model}

In this section we present a finite extensive-form multistage game with incomplete information, multiple priors and perfect recall. The definition is similar to Hanany, Klibanoff, and Mukerji (2018), but instead of smooth ambiguity aversion players are faced with imprecise probabilistic information and maxmin preferences. We will show later that given this imprecise probabilistic information and the information structure of the game each

\footnotetext{
${ }^{6}$ Sarin and Wakker (1998) do not use the term rectangularity. Instead they use the term reduced family of probability measures for the rectangular hull as defined in Epstein and Schneider (2003).
} 
player construct a subjective set of prior beliefs. Given this beliefs each player evaluate a strategy by using maxmin expected utility (MEU).

Definition 1 A finite extensive-form multistage game with incomplete information, perfect recall and multiple priors, $\Gamma$, is a tuple $\left(N, H,\left(\mathcal{I}_{i}\right)_{i \in N},\left(u_{i}\right)_{i \in N}, \mathcal{P}\right)$ where

- $N$ is a finite set of players

- $H$ is a finite set of histories with elements $h=\left(h_{-1},\left(h_{0, i}\right)_{i \in N}, \ldots,\left(h_{T, i}\right)_{i \in N}\right)$. For $0 \leq t \leq T+1$ let $H^{t}:=\left\{h^{t}:=\left(h_{-1},\left(h_{0, i}\right)_{i \in N}, \ldots,\left(h_{t-1, i}\right)_{i \in N}\right) \mid h \in H\right\}$ be the set of partial histories up to but not including t. $\mathcal{H}:=\{\emptyset\} \cup \bigcup_{0 \leq t \leq T+1} H^{t}$ is the set of all partial histories. Given a partial history $\eta \in \mathcal{H}$ and $-1 \leq \bar{t} \leq|\eta|-1$, $\eta_{t}$ denotes the element of $\eta$ at stage $t$ and $\eta_{t, i}$ player $i$ 's component of $\eta_{t}$.

For each $i \in N, 0 \leq t \leq T+1$ and $h^{t} \in H^{t}, A_{i}\left(h^{t}\right):=\left\{\hat{h}_{t, i} \mid \hat{h} \in H, \hat{h}^{t}=h^{t}\right\}$ is the set of actions available to player $i$ at $h^{t}$. The set of types is $H^{0}$.

- $\mathcal{I}_{i}:=\bigcup_{0 \leq t \leq T} \mathcal{I}_{i}^{t}$ are the information sets for player $i$, where each $\mathcal{I}_{i}^{t}$ is a partition of $H^{t}$ such that for all $\eta^{t}, \hat{\eta}^{t} \in H^{t}, \hat{\eta}^{t} \in I_{i}\left(\eta^{t}\right)$ implies $A_{i}\left(\eta^{t}\right)=A_{i}\left(\hat{\eta}^{t}\right)$, where $I_{i}\left(\eta^{t}\right)$ is the unique element of $\mathcal{I}_{i}^{t}$ such that $\eta^{t} \in I_{i}\left(\eta^{t}\right)$. For $0 \leq t \leq T$ and $\eta^{t} \in N$, $R_{i}\left(\eta^{t}\right):=\left(\left(I_{i}\left(\eta^{s}\right), \eta_{s, i}^{t}\right)_{0 \leq s<t}, I_{i}\left(\eta^{t}\right)\right)$ is the ordered list of information sets $i$ encounters and the action $i$ takes under partial history $\eta^{t}$. The game satisfies perfect recall in that for each player $i, 0 \leq t \leq T$ and $\eta^{t}, \hat{\eta}^{t} \in H^{t}, I_{i}\left(\eta^{t}\right)=I_{i}\left(\hat{\eta}^{t}\right)$ implies $R_{i}\left(\eta^{t}\right)=R_{i}\left(\hat{\eta}^{t}\right)$.

- $u_{i}: H \rightarrow \mathbb{R}$ is the utility of player $i$ given a history.

- $\mathcal{P} \subset \Delta H^{0}$ is the set of imprecise probabilistic information and homogeneous across all players. We assume that $\mathcal{P}$ is compact and all $\pi \in \mathcal{P}$ have full support, i.e. $\pi\left(h^{0}\right)>0$ for all $h^{0} \in H^{0}$ and all $\pi \in \mathcal{P}$.

The definition above allows for imperfectly observed actions as well as for private information about types or states. The multistage structure assumes that each player chooses an action at each stage. Since $A_{i}\left(h^{t}\right)$ can be singleton, this assumption is not restrictive and sequential play can be modeled as well.

The only difference compared to the standard setting without ambiguity is the last bullet point. Players do not have an exact distribution over types. This imprecise information is covered by a set of possible distributions $\mathcal{P}$. If $\mathcal{P}$ is singleton there is no ambiguity and the game reduce to the standard version without ambiguity. The compactness assumption on $\mathcal{P}$ ensures the existence of a worst case belief. Full support ensures that out-of-equilibrium path only occurs because of non-completely mixed strategies. Therefore, for completely mixed strategies Bayes' rule is always well defined.

At each stage conditional on their information set $I_{i}^{t}$ players choose a distribution over their actions which are available at $I_{i}^{t}$. A strategy profile denotes these distributions for each player and information set. 
Definition 2 A (behavioural) strategy for player $i$ in a game $\Gamma$ is a function $\sigma_{i}$ such that $\sigma_{i}\left(I_{i}^{t}\right) \in \Delta\left(A_{i}\left(I_{i}^{t}\right)\right)$ for each $I_{i}^{t} \in \mathcal{I}_{i}^{t}$, where $\Delta\left(A_{i}\left(I_{i}^{t}\right)\right)$ denotes the set of all probability vectors over $A_{i}\left(I_{i}^{t}\right)$.

Furthermore, let $\Sigma_{i}$ denote the set of all strategies for player $i, \sigma:=\left(\sigma_{i}\right)_{i \in N}$ a strategy profile and $\sigma_{-i}:=\left(\sigma_{j}\right)_{j \neq i}$ the strategies of all opponents of $i$.

A strategy profile induces a transition probability with which a particular (partial) history occurs. For a given strategy profile $\sigma$, a history $h$ and $0 \leq r \leq t \leq T+1$ the probability of reaching $h^{t}$ starting from $h^{r}$ is $p_{\sigma}\left(h^{t} \mid h^{r}\right):=\prod_{j \in N} \prod_{r \leq s<t} \sigma_{j}\left(I_{j}\left(h^{s}\right)\right)\left(h_{s, j}\right)$. It will be useful to split $p_{\sigma}\left(h^{t} \mid h^{r}\right)$ in one part that only depends on the agent himself and another part that represents the actions of all opponents. We define $p_{\sigma_{i}}\left(h^{t} \mid h^{r}\right):=\prod_{r<s<t} \sigma_{i}\left(I_{i}\left(h^{s}\right)\right)\left(h_{s, i}\right)$ and $p_{\sigma_{-i}}\left(h^{t} \mid h^{r}\right):=\prod_{j \neq i} \prod_{r \leq s<t} \sigma_{j}\left(I_{j}\left(h^{s}\right)\right)\left(h_{s, j}\right)$. Hence, $p_{\sigma_{i}}\left(h^{t} \mid h^{r}\right) p_{\sigma_{-i}}\left(h^{t} \mid h^{r}\right)=p_{\sigma}\left(h^{t} \mid h^{r}\right)$.

\subsection{Dynamic Inconsistency}

Multiple priors can lead to dynamically inconsistent behavior. To illustrate dynamic inconsistency we repeat the 3 player example from Aryal and Stauber (2014). ${ }^{7}$ We will use this example as a running example in the next sections to illustrate notation and results.

Running Example The game, depicted in Figure 1, shows that ambiguity and multiple prior can lead to dynamically inconsistent behavior. There are two players, player 1 and

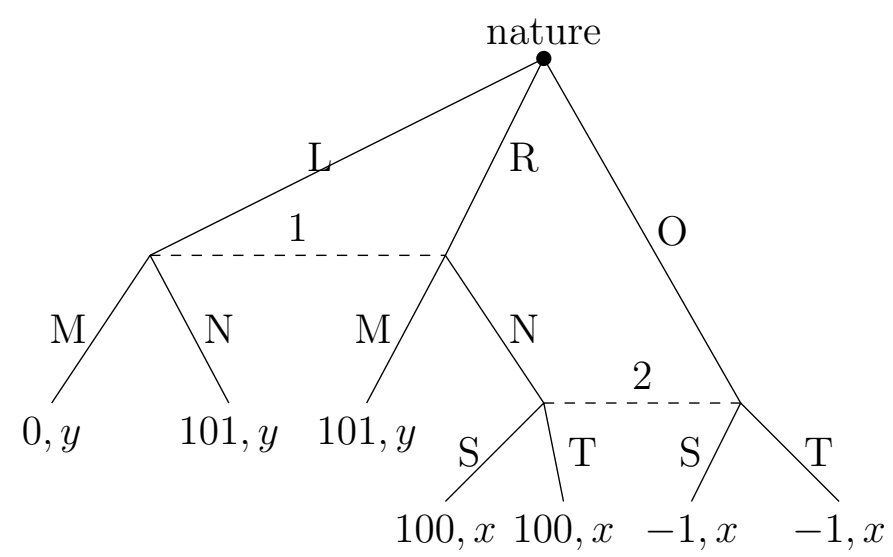

Figure 1: Aryal and Stauber 3 player game

player 2. Before they play an action nature chooses the state $L, R$ or $O$. Let $l, r$ and $o$ be the probability of $L, R$ and $O$, respectively. The imprecise probabilistic information is given by an $\epsilon$-contamination of the distribution that assigns probability 1 to $R$, i.e.

\footnotetext{
${ }^{7}$ To fit our definition of multi-stage games one would had to include constant actions for player 2 at the information set of player 1 and a constant action for player 1 at the information set of player 2 . Since this does not change the results of the example we skip these constant actions due to notational convenience.
} 
$(l, r, o)=(0,1,0)$. This represents a setting where the probability, that $R$ is chosen is very high. However, there is a small probability $\epsilon \in[0,1]$ that any distribution could be true as well. Let $\Delta$ be the set of all probability distributions over $\{L, R, O\}$. The imprecise probabilistic information is given by the set ${ }^{8}$

$$
\mathcal{P}=\{(1-\epsilon)(0,1,0)+\epsilon(l, r, o) \text { s.t. }(l, r, o) \in \Delta\} .
$$

To illustrate the problem of dynamic consistency assume that $\mathcal{P}$ represent the beliefs of player 1 .

After the choice of nature player 1 can observe if the state is $O$ or not. If the state is not $O$ player 1 can choose between $N$ and $M$. If the state is $O$ or $R$ and player 1 played $N$, player 2 can choose an action without knowing which of the two cases is true. The information sets of both players are depicted by dashed lines. For the moment let us concentrate on player 1. His payoffs are independent of the strategy of player 2. He gets his lowest payoff, -1, if the state is $O$, no matter if player 2 plays $S$ or $T$. Therefore, his ex-ante worst case belief gives the highest probability to $O$, i.e. the ex-ante worst case belief given $\mathcal{P}$ is $(0,1-\epsilon, \epsilon)$. Since the probability of $L$ is 0 he gets 101 if he plays $M$ and 100 if he plays $N$ (independent of the strategy of player 2). Therefore, ex-ante his optimal strategy is playing $M$ with probability 1.

Now we check if player 1 has an incentive to deviate from his optimal ex-ante strategy after observing that the state is not $O$. Updating $\mathcal{P}$ prior by prior using Bayes' rule and conditioning on the event $\{L, R\}$ leads to the following set of updated beliefs

$$
\operatorname{Bay}(\mathcal{P} \mid\{L, R\})=\{(l, r)=(1-r, r) \text { s.t. } r \in[1-\epsilon, 1]\} .
$$

His interim worst case belief depends on his own strategy. Playing $M$ with probability 1 would leads to a payoff of 0 if the state is $L$ and a payoff of 101 if the state is $R$. Given this strategy his worst case belief would be $(\epsilon, 1-\epsilon)$. Given this belief playing $M$ with probability 1 is not longer optimal. On the other hand playing $N$ with probability 1 leads to a payoff of 101 or 100 if the state is $L$ or $R$, respectively. Hence, the worst case belief if he plays $N$ with probability 1 is $(0,1)$. But for this belief playing $M$ with probability 1 is optimal. One can show that for $\epsilon>\frac{1}{102}$ the optimal interim strategy of player 1 is a mixed strategy with probability $\frac{1}{102}<1$ for $M$.

\footnotetext{
${ }^{8} \mathcal{P}$ does not satisfy the full support assumption stated in Definition 1 . Formally the full support assumption is needed to guaranty that the probability of reaching an information set is 0 if and only if all partial histories leading to this information set have probability 0 because of the played strategy profile. Hence, if the probability of reaching an information set is 0 for some prior belief $\pi \in \mathcal{P}$ then it is 0 for all prior beliefs. Due to the $\epsilon$-contamination structure of $\mathcal{P}$ the probability of $R$ is at least $1-\epsilon$ for all prior beliefs. Therefore the information set of player 1 has always positive probability. If player 1 plays $N$ with probability 0 the information set of player 2 will not be reached if $O$ has probability 0 . Hence, in this case it depends on the prior belief if the probability of reaching the information set of player 2 is strictly positive. Assuming a prior belief set $\mathcal{P}$ satisfying the full support assumption would lead to the same results as long as the minimum probability of $L$ is small enough (i.e. smaller then $\frac{1}{102}$ ). Since the payoff of player 2 is independent of his own actions and since the payoff of player 1 is constant w.r.t. to the action chosen by player 2 we skip the full support assumption due to notational convenience.
} 
Hence, player 1 is dynamically inconsistently and plays a different strategy after observing that the state is not $O$. For detailed calculations see Aryal and Stauber (2014) or Section B.1.1 in the Appendix.

The example above shows that new information can change beliefs in such a way that the optimal strategy given the beliefs changes as well. This leads to dynamically inconsistent behavior. In decision theoretic settings an important property of set of distributions or beliefs to ensure dynamic consistency is rectangularity, or sometimes called stability under pasting introduced by Epstein and Schneider (2003) and Sarin and Wakker (1998).

Rectangularity can be interpreted as a generalization of the law of iterated expectation. It captures the idea of decomposing any probability measure into its conditionals and marginals. Therefore, agents take their worst case beliefs at some interim stage into account when they choose their ex-ante worst case belief. Since the structure of the game, as described in Definition 1, is known to each player, each player knows the possible information sets for each stage. Therefore, for each information sets each player can calculate his set of updated beliefs. Knowing these possible sets of updated beliefs he construct his set of prior beliefs in a rational way that is crucial for dynamic consistency. This is given by constructing a set of prior beliefs such that the belief system is stable under pasting (or rectangular).

To define rectangularity we have to consider that the information that each player receives are actions played by the opponents. Furthermore, usually each player is faced with different information sets and knows the actions chosen by himself. This implies that in a game each player receives different information than his opponents. The idea of rectangularity is to take possible future information into account, when choosing the ex-ante beliefs. Together with heterogeneous information this leads to a heterogeneous prior belief set for each agent and is the main difference between rectangularity in decision theoretic settings and games.

In the next section we formulate the definition of beliefs in our setting and give a formal definition of rectangularity.

\section{$2.2 \quad$ Beliefs}

In the game theoretic setting the information is represented by the information sets $\mathcal{I}$. A probability distribution or belief at one of these information set is a probability distribution over the partial histories leading to this information set. During the game players observe actions played by their opponents. These observations lead to new information about the types but also about strategies of the opponents. Hence, in the standard game theoretic setting the set over which a player forms his beliefs changes in each period $t$. To define a belief system which is rectangular, we need a sequence of partitions which represents the information flow of the game and is based on the same set at each period.

Given the game defined in Definition 1 we can define a sequence of partitions which represents the information flow of the game. 
Definition 3 Given the set of histories $\mathcal{H}$ and the information sets $\mathcal{I}$ let $\left(\mathcal{F}_{i}^{t}\right)_{t=0, \ldots, T+1}$ denote the sequence of information partitions of player $i$, where

$$
\begin{aligned}
& \mathcal{F}_{i}^{0}=H \\
& \mathcal{F}_{i}^{t}=\left\{\left\{h \in H \text { s.t } h^{t} \in I_{i}^{t}\right\}_{I_{i}^{t} \in \mathcal{I}_{i}^{t}}\right\} .
\end{aligned}
$$

Since there is a one-to-one relation between the elements $F_{i}^{t}$ of $\mathcal{F}_{i}^{t}$ and the information sets $I_{i}^{t} \in \mathcal{I}_{i}^{t}$, we sometimes call $F_{i}^{t}$ an information set.

Furthermore, new information is influenced by strategic aspects in game theoretic settings. In decision theory new information occurs usually as a exogeneously given signal. In games the signals are observable actions of the opponents. Therefore, the strategy of the opponents influence the information that a player observes. This motivates the following definition of beliefs. For additional motivation see Example 3 in the Appendix.

Fix a player $i$ and a strategy profile $\sigma_{-i}$. First we construct the set of prior beliefs induced by the partition $\left(\mathcal{F}_{i}^{t}\right)_{t=0, \ldots, T+1}$, the imprecise probabilistic information $\mathcal{P}$ and $\sigma_{-i}$.

Definition 4 Given $\left(\mathcal{F}_{i}^{t}\right)_{t=0, \ldots, T+1}, \mathcal{P}$ and a strategy profile $\sigma_{-i}$ let $\Phi_{\sigma_{-i}}^{0}$ denote the prior belief set of player $i$ with

$$
\Phi_{\sigma_{-i}}^{0}=\left\{\phi \in[0,1]^{H} \text { s.t. } \phi(h)=p_{\sigma_{-i}}\left(h \mid h^{0}\right) \pi\left(h^{0}\right) \text { with } \pi \in \mathcal{P}\right\} .
$$

Note that $\Phi_{\sigma_{-i}}^{0} \nsubseteq \Delta H$ since $\phi \in \Phi_{\sigma_{-i}}^{0}$ is in general not normalized to 1 . One can easily normalize $\phi$ by dividing through the constant $C_{\sigma_{-i}, \pi}=\sum_{h \in H} p_{\sigma_{-i}}\left(h \mid h^{0}\right) \pi\left(h^{0}\right)$. However, since normalization is not important for the further considerations (see Remark 3) we skip the normalization due to notational convenience.

As in standard game theory settings we define a system of beliefs as a tuple that defines a belief for each information set of each player.

Definition 5 A belief system $\Psi=\left(\Psi_{i}^{t}\right)_{i \in N, t=0, \ldots, T+1}$ consists of a set of belief $\Psi_{i}^{t}\left(F_{i}^{t}\right) \subseteq \Delta H$ for each information set $F_{i}^{t}$ at stages $t>0$ and $\Psi_{i}^{0}=\Phi_{\sigma_{-i}}^{0}$ for each player $i$.

To illustrate the definitions above we come back to our running example

Running Example (cont.) We denote with LM the history where nature chooses type $L$ and player 1 plays $M$. All histories are denoted in a similar way. Furthermore, denote the probability with which player 1 plays $N$ with $n$ and similarly all probabilities of a certain action with the corresponding lower case. The set of all histories $H$ is then given by

$$
H=\{L M, L N, R M, R N S, R N T, O S, O T\} .
$$

At the ex-ante stage player 1 and 2 have no information about the states. Therefore, their information partition at the ex-ante stage consist only of one element, the set of all histories $H$.

$$
\mathcal{F}_{i}^{0}=H
$$


At the interim stage player 1 can observe if the state is $O$ or not. His information set consists of three elements. The first set contains all histories starting at $L$ or $R$. The second and third set represent the case where player 1 learns that the state is $O$.

$$
\mathcal{F}_{1}^{1}=\left\{F_{1,1}^{1}, F_{1,2}^{1}, F_{1,3}^{1}\right\}=\{\{L M, L N, R M, R N S, R N T\},\{O S\},\{O T\}\} .
$$

Similarly player 2's interim information partition consists of the set that contains all histories starting from $O$ and histories where the state is $R$ and player 1 plays $N$ and the sets where he learns the exact history.

$$
\mathcal{F}_{2}^{1}=\left\{F_{2,1}^{1}, F_{2,2}^{1}, F_{2,3}^{1}, F_{2,4}^{1}\right\}=\{\{R N S, R N T, O S, O T\},\{L M\},\{L N\},\{R M\}\} .
$$

A strategy $\sigma_{-1}=(s, t)$ of player 2 induces a transition probability $p_{\sigma_{-1}}(\cdot \mid \cdot)$ for each history $h \in H$ which is independent of his own strategy. Multiplying the imprecise probabilistic information with the transition probability, $p_{\sigma_{-1}}(\cdot \mid \cdot)$, induced by $\sigma_{-1}=(s, t)$ leads to the following set of ex-ante beliefs for player 1

$$
\Phi_{(s, t)}^{0}=\{(l, l, r, r s, r t, o s, o t) \text { s.t. }(l, r, o) \in \mathcal{P}\} .
$$

Similarly, the ex-ante belief set of player 2 given strategy $\sigma_{\sigma_{-2}}=(m, n)$ of player 1 is

$$
\Phi_{(m, n)}^{0}=\{(l m, l n, r m, r n, r n, o, o) \text { s.t. }(l, r, o) \in \mathcal{P}\} .
$$

\subsubsection{Rectangularity}

Given the above notation and definitions, we formulate the formal definition of stability under pasting. As already mentioned stability under pasting is a generalization of the law of iterative expectation.

Let us first look at the case without ambiguity, i.e. there exist only one prior belief $\phi$, and assume that Bayes' rule is always well defined. For each information set $F_{i}^{1}$ in the next stage Bayesian updating leads to an updated belief $\tilde{\phi}_{F_{i}}$. Roughly speaking, Bayes' rule is defined such that the denominator of Bayes' rule equals the marginal belief of reaching the information set on which we update the prior belief. Hence, multiplying (or pasting) the updated belief given an information set $F_{i}^{1}$ with the marginal belief of reaching this information set leads to the prior belief restricted to $F_{i}^{1}$. This holds for any information set and therefore summation over all information sets leads to the prior belief on $H$, i.e.

$$
\phi(h)=\sum_{F_{i}^{1} \in \mathcal{F}_{i}^{1}} \phi\left(F_{i}^{1}\right) \tilde{\phi}_{F_{i}^{1}}(h) .
$$

Now, we generalize this property to an ambiguous setting. With ambiguity players have a set of prior beliefs. Stability under pasting states that we can take any updated and marginal belief (even if they are not derived from the same prior belief) and the pasting is still an element of the prior belief set. 
Definition 6 For beliefs $\phi \in \Psi_{i}^{t-1}\left(F_{i}^{t-1}\right)$ and $\tilde{\phi}=\left(\tilde{\phi}_{F_{i}^{t}}\right)_{F_{i}^{t} \in \mathcal{F}_{i}^{t}}$ with $\tilde{\phi}_{F_{i}^{t}} \in \Psi_{i}^{t}\left(F_{i}^{t}\right)$ the pasting of marginal and updated belief, $\phi \circ \tilde{\phi}$, is defined as

$$
\phi \circ \tilde{\phi}(\cdot)=\sum_{F_{i}^{t} \in \mathcal{F}_{i}^{t}} \phi\left(F_{i}^{t}\right) \tilde{\phi}_{F_{i}^{t}}(\cdot) .
$$

The pasting of $\Psi_{i}^{t-1}\left(F_{i}^{t-1}\right)$ and $\left(\Psi_{i}^{t}\left(F_{i}^{t}\right)\right)_{F_{i}^{t} \in \mathcal{F}_{i}^{t}}$ is defined as the set consisting of pasting each element of $\Psi_{i}^{t-1}\left(F_{i}^{t-1}\right)$ with each element of $\left(\Psi_{i}^{t}\left(F_{i}^{t}\right)\right)_{F_{i}^{t} \in \mathcal{F}_{i}^{t}}$, i.e.

$$
\Psi_{i}^{t-1}\left(F_{i}^{t-1}\right) \circ\left(\Psi_{i}^{t}\left(F_{i}^{t}\right)\right)_{F_{i}^{t} \in \mathcal{F}_{i}^{t}}=\left\{\phi \circ \tilde{\phi} \text { with } \phi \in \Psi_{i}^{t-1}\left(F_{i}^{t-1}\right) \text { and } \tilde{\phi}_{F_{i}^{t}} \in \Psi_{i}^{t}\left(F_{i}^{t}\right)\right\} \text {. }
$$

A set of beliefs $\Psi_{i}^{t-1}\left(F_{i}^{t-1}\right)$ is called rectangular (stable under pasting) if

$$
\Psi_{i}^{t-1}\left(F_{i}^{t-1}\right) \circ\left(\Psi_{i}^{t}\left(F_{i}^{t}\right)\right)_{F_{i}^{t} \in \mathcal{F}_{i}^{t}}=\Psi_{i}^{t-1}\left(F_{i}^{t-1}\right) .
$$

A belief system $\Psi$ is called stable under pasting (rectangular) if $\Psi_{i}^{t-1}\left(F_{i}^{t-1}\right)$ is stable under pasting for all $F_{i}^{t} \in \mathcal{F}_{i}^{t}, i \in N$ and $t=1, \ldots T+1$.

By the intuition given above, without ambiguity, rectangularity should follow from Bayesian updating. The following remark shows that this is indeed true.

Remark 1 Let $\Psi_{i}^{0}$ be singleton and $\phi\left(F_{i}^{t}\right)>0$ for all $F_{i}^{t}$. Then Bayes' rule is always well defined and stability under pasting is equivalent to Bayes' rule. To see this take into account that since $\Psi_{i}^{0}$ is singleton, $\Psi_{i}^{t}\left(F_{i}^{t}\right)$ are singleton as well. Denote by $\bar{F}_{i}^{t}$ the element of the partition $\mathcal{F}_{i}^{t}$ which contains $h$. First we show that Bayes' rule implies stability under pasting.

$$
\begin{aligned}
\phi \circ \tilde{\phi}(h) & =\sum_{F_{i}^{t} \in \mathcal{F}_{i}^{t}} \phi\left(F_{i}^{t}\right) \tilde{\phi}_{F_{i}^{t}}(h) \\
& =\phi\left(\bar{F}_{i}^{t}\right) \tilde{\phi}_{\bar{F}_{i}^{t}}(h) \\
& =\phi\left(\bar{F}_{i}^{t}\right) \frac{\phi(h)}{\phi\left(\bar{F}_{i}^{t}\right)} \\
& =\phi(h) .
\end{aligned}
$$

The other direction follows by similar calculations since $\phi\left(\bar{F}_{i}^{t}\right)>0$.

$$
\begin{aligned}
\phi(h) & =\phi \circ \tilde{\phi}(h) \\
& =\phi\left(\bar{F}_{i}^{t}\right) \tilde{\phi}_{\bar{F}_{i}^{t}}(h) \\
\Leftrightarrow \quad \tilde{\phi}_{\bar{F}_{i}^{t}}(h) & =\frac{\phi(h)}{\phi\left(\bar{F}_{i}^{t}\right)}
\end{aligned}
$$

Furthermore, rectangularity preserve some nice properties. Remark 2 shows that the Bayesian update of $\phi \circ \tilde{\phi}$ equals $\tilde{\phi}$ and the marginal probability of a pasting $\phi \circ \tilde{\phi}$ equals 
the marginal probability of $\phi$. We will see that these properties are very useful when we explain the construction of a belief system which is stable under pasting.

Remark 2 Let $\Psi_{i}^{t-1}\left(F_{i}^{t-1}\right)$ be stable under pasting. Then for any $\phi \in \Psi_{i}^{t-1}\left(F_{i}^{t-1}\right)$ there exist some $\phi^{\prime} \in \Psi_{i}^{t-1}\left(F_{i}^{t-1}\right)$ and $\tilde{\phi}=\left(\tilde{\phi}_{F_{i}^{t}}\right)_{F_{i}^{t} \in \mathcal{F}_{i}^{t}} \in\left(\Psi_{i}^{t}\left(F_{i}^{t}\right)\right)_{F_{i}^{t} \in \mathcal{F}_{i}^{t}}$ such that

$$
\phi(\bar{h})=\phi^{\prime} \circ \tilde{\phi}(\bar{h})=\sum_{F_{i}^{t} \in \mathcal{F}_{i}^{t}} \phi^{\prime}\left(F_{i}^{t}\right) \tilde{\phi}_{F_{i}^{t}}(\bar{h}) \text {. }
$$

Let $\bar{F}_{i}^{t}$ denote the element of the partition which contains $\bar{h}$. Then $\phi(\bar{h})=\phi^{\prime}\left(\bar{F}_{i}^{t}\right) \tilde{\phi}_{\bar{F}_{i}^{t}}(\bar{h})$.

- The Bayesian update of $\phi$ given $\bar{F}_{i}^{t}$ is

$$
\begin{aligned}
\operatorname{Bay}\left(\phi \mid \bar{F}_{i}^{t}\right)(\bar{h}) & =\frac{\phi(\bar{h})}{\sum_{h \in \bar{F}_{i}^{t}} \phi(h)} \\
& =\frac{\sum_{F_{i}^{t}} \phi^{\prime}\left(F_{i}^{t}\right) \tilde{\phi}_{F_{i}^{t}}(\bar{h})}{\sum_{h \in \bar{F}_{i}^{t}} \sum_{F_{i}^{t}} \phi^{\prime}\left(F_{i}^{t}\right) \tilde{\phi}_{F_{i}^{t}}(h)} \\
& =\frac{\phi^{\prime}\left(\bar{F}_{i}^{t}\right) \tilde{\phi}_{\bar{F}_{i}^{t}}(\bar{h})}{\sum_{h \in \bar{F}_{i}^{t}} \phi^{\prime}\left(\bar{F}_{i}^{t}\right) \tilde{\phi}_{\bar{F}_{i}^{t}}(h)} \\
& =\frac{\phi^{\prime}\left(\bar{F}_{i}^{t}\right) \tilde{\phi}_{\bar{F}_{i}^{t}}(\bar{h})}{\phi^{\prime}\left(\bar{F}_{i}^{t}\right) \sum_{h \in \bar{F}_{i}^{t}} \tilde{\phi}_{\bar{F}_{i}^{t}}(h)} \\
& =\frac{\tilde{\phi}_{\bar{F}_{i}^{t}}(\bar{h})}{\sum_{h \in \bar{F}_{i}^{t}} \tilde{\phi}_{\bar{F}_{i}^{t}}(h)} \\
& =\tilde{\phi}_{\bar{F}_{i}^{t}}(\bar{h})
\end{aligned}
$$

where the last equality follows since $\sum_{h \in \bar{F}_{i}^{t}} \tilde{\phi}_{\bar{F}_{i}^{t}}(h)=1$ for all $t>0 .{ }^{9}$

- The marginal distribution is

$$
\begin{aligned}
\sum_{h \in \bar{F}_{i}^{t}} \phi(h) & =\sum_{h \in \bar{F}_{i}^{t}} \sum_{F_{i}^{t}} \phi^{\prime}\left(F_{i}^{t}\right) \tilde{\phi}_{F_{i}^{t}}(h) \\
& =\sum_{h \in \bar{F}_{i}^{t}} \phi^{\prime}\left(\bar{F}_{i}^{t}\right) \tilde{\phi}_{\bar{F}_{i}^{t}}(h) \\
& =\phi^{\prime}\left(\bar{F}_{i}^{t}\right) \underbrace{\sum_{h \in \bar{F}_{i}^{t}} \tilde{\phi}_{\bar{F}_{i}^{t}}(h)}_{=1} \\
& =\phi^{\prime}\left(\bar{F}_{i}^{t}\right) .
\end{aligned}
$$

${ }^{9}$ Note that Bayes' rule implies that $\tilde{\phi}_{\bar{F}_{i}^{t}}$ is normalized to 1 for all $t>0$. 
Hence, marginal and updated distribution of a distribution of a belief set which is stable under pasting, coincide with the marginal and updated distribution from which it is constructed.

The decision theoretic papers using rectangularity and cited above, show that a rectangular belief system can always be constructed in the following way. First, given the prior belief set and the information structure one can calculate the prior by prior Bayesian updates for all information sets. Then, one proceeds by backward induction and constructs a rectangular belief set by pasting marginal and updated beliefs. The belief sets constructed like this are the smallest rectangular sets which contain the original belief sets. Therefore, they are called the rectangular hulls of the original belief sets. The same method can be used here to get a belief system which is stable under pasting for completely mixed $\sigma_{-i}$. When $\sigma_{-i}$ is completely mixed, Bayes' rule is always well defined and we can derive the prior by prior Bayesian update of $\Phi_{\sigma_{-i}}^{0}$ for each stage and each information set. Denote with $\Phi_{\sigma_{-i}}^{t-1}\left(F_{i}^{t-1}\right)$ the Bayesian update of $\Phi_{\sigma_{-i}}^{0}$ at $t-1$ given the information set $F_{i}^{t-1}$ and similarly with $\Phi_{\sigma_{-i}}^{t}\left(F_{i}^{t}\right)$ the Bayesian update at $t$ given $F_{i}^{t} \cdot{ }^{10}$ The rectangular hull, $\operatorname{rect}\left(\Phi_{\sigma_{-i}}^{t-1}\left(F_{i}^{t-1}\right)\right)$, is given by the pasting of $\Phi_{\sigma_{-i}}^{t-1}\left(F_{i}^{t-1}\right)$ and $\Phi_{\sigma_{-i}}^{t}$, i.e.

$$
\operatorname{rect}\left(\Phi_{\sigma_{-i}}^{t-1}\right)=\left\{\phi \circ \tilde{\phi} \text { with } \phi \in \Phi_{\sigma_{-i}}^{t-1}\left(F_{i}^{t-1}\right) \text { and } \tilde{\phi}_{F_{i}^{t}} \in \Phi_{\sigma_{-i}}^{t}\left(F_{i}^{t}\right) \quad \forall F_{i}^{t} \in \mathcal{F}_{i}^{t}\right\}
$$

Remark 2 shows that the set of Bayesian updates of $\operatorname{rect}\left(\Phi_{\sigma_{-i}}^{t-1}\right)$ coincide with the set of Bayesian updates of $\Phi_{\sigma_{-i}}^{t-1}$. Therefore, $\operatorname{rect}\left(\Phi_{\sigma_{-i}}^{t-1}\right)$ is stable under pasting by construction. This method holds for any arbitrary $t$. Hence, starting with the last two periods, $T-1$ and $T$, and proceeding by backward induction we can always close a prior set $\Phi_{\sigma_{-i}}^{0}$ under pasting and $\operatorname{rect}\left(\Phi_{\sigma_{-i}}^{0}\right)$ is the smallest set containing $\Phi_{\sigma_{-i}}^{0}$ that is stable under pasting. Furthermore, the construction induces that the Bayesian updates of $\operatorname{rect}\left(\Phi_{\sigma_{-i}}^{0}\right)$ are stable under pasting for any information set.

If $\sigma_{-i}$ is not completely mixed, there can exists information sets such that the marginal probability of reaching this information sets is 0 . Let $\bar{F}_{i}^{t}$ be an information set such that there exists $\phi \in \Phi_{\sigma_{-i}}^{0}$ with $\phi\left(\bar{F}_{i}^{t}\right)=0$. The full support assumption of $\mathcal{P}$ implies that $\phi^{\prime}\left(\bar{F}_{i}^{t}\right)=0$ for all $\phi^{\prime} \in \Phi_{\sigma_{-i}}^{0}$. Let $\bar{F}_{i}^{t-1}$ be information set that precedes $\bar{F}_{i}^{t}$, i.e. $\bar{F}_{i}^{t} \subseteq \bar{F}_{i}^{t-1}$ and without loss of generality let $\phi\left(\bar{F}_{i}^{t-1}\right)>0 .{ }^{11}$ Furthermore, perfect recall implies that all information sets that are reachable from $\bar{F}_{i}^{t}$ have probability 0 as well. For information sets with probability 0 Bayes' rule is not well defined. The construction of the rectangular hull described above can be generalized as follows. For all information sets with positive probability the set of updated beliefs are derived by Bayes' rule. For information sets with probability 0 players can choose an arbitrary compact set of updated beliefs. Then, the

\footnotetext{
${ }^{10} \mathrm{We}$ assume that the Bayesian update is a probability distribution over the whole set of full histories $H$ such that histories which are not an element of the observed information set have probability 0.

${ }^{11}$ If $\phi\left(\bar{F}_{i}^{t-1}\right)=0$ we can replace $\bar{F}_{i}^{t}$ by $\bar{F}_{i}^{t-1}$ and check if the probability of the information set preceding $\bar{F}_{i}^{t-1}$ has positive probability. Repeating this leads to an information set with probability 0 such that the preceding information set has strictly positive probability.
} 
rectangular hull is constructed by backward induction as described above. The construction may change the set of beliefs at information sets with 0 probability. But the construction of the rectangular hull of the belief set at $\bar{F}_{i}^{t-1}$ is not influenced by the belief set at $\bar{F}_{i}^{t}$, since the marginal probability of $\bar{F}_{i}^{t}$ is 0 . Therefore, the arbitrary choice of updated belief sets at information sets with probability 0 does not influence the construction of the rectangular hull and we use the notation $\operatorname{rect}\left(\Phi_{\sigma_{-i}}^{t}\right)$ for any strategy $\sigma_{-i} \in \Sigma_{-i}$.

We mentioned above that in general ex ante beliefs $\phi \in \Phi_{\sigma_{-i}}^{0}$ are not normalized to 1 . The following remark shows why this does not influence the construction of the rectangular hull.

Remark 3 First consider the fact that each belief $\tilde{\phi} \in \operatorname{Bay}\left(\Phi_{\sigma_{-i}}^{0} \mid \cdot\right)$ is normalized to 1 by the structure of Bayesian updating. Hence, the non-normalization of $\Phi_{\sigma_{i}}^{0}$ could only influence the rectangular hull of $\Phi_{\sigma_{i}}^{0}$. Lets compare the pasting of updated belief $\tilde{\phi}$ with a non-normalized prior $\phi$ and the pasting of $\tilde{\phi}$ with a normalized prior $\phi^{\prime}=\frac{1}{C_{\sigma_{-i}, \pi}} \phi$.

$$
\phi^{\prime} \circ \tilde{\phi}(\cdot)=\sum_{F_{i}^{1} \in \mathcal{F}_{i}^{1}} \frac{1}{C_{\sigma_{-i}, \pi}} \phi\left(F_{i}^{1}\right) \tilde{\phi}(\cdot) .
$$

Since $C_{\sigma_{-i}, \pi}$ is constant across all $h \in F_{i}^{1}$ it follows

$$
\phi^{\prime} \circ \tilde{\phi}(\cdot)=\frac{1}{C_{\sigma_{-i}, \pi}}(\phi \circ \tilde{\phi}(\cdot)) .
$$

Therefore, normalizing $\Phi_{\sigma_{-i}}^{0}$ would lead to a normalization of $\operatorname{rect}\left(\Phi_{\sigma_{-i}}^{0}\right)$ but would not change the structure of $\operatorname{rect}\left(\Phi_{\sigma_{-i}}^{0}\right)$.

To illustrate the construction of an rectangular prior set we come back to our running example.

Running Example (cont.) We have already shown that

$$
\begin{aligned}
H & =\{L M, L N, R M, R N S, R N T, O S, O T\}, \\
\mathcal{F}_{i}^{0} & =H, \\
\mathcal{F}_{1}^{1} & =\left\{F_{1,1}^{1}, F_{1,2}^{1}, F_{1,3}^{1}\right\}=\{\{L M, L N, R M, R N S, R N T\},\{O S\},\{O T\}\}, \\
\mathcal{F}_{2}^{1} & =\left\{F_{2,1}^{1}, F_{2,2}^{1}, F_{2,3}^{1}, F_{2,4}^{1}\right\}=\{\{R N S, R N T, O S, O T\},\{L M\},\{L N\},\{R M\}\}, \\
\Phi_{(s, t)}^{0} & =\{(l, l, r, r s, r t, o s, \text { ot }) \text { s.t. }(l, r, o) \in \mathcal{P}\}, \\
\Phi_{(m, n)}^{0} & =\{(l m, l n, r m, r n, r n, o, o) \text { s.t. }(l, r, o) \in \mathcal{P}\} .
\end{aligned}
$$

To construct the rectangular hull of $\Phi_{(s, t)}^{0}$ we need the marginal and updated beliefs of player 1. The marginal beliefs for an arbitrary $\phi \in \Phi_{(s, t)}^{0}$ of the information sets of player 1 are

$$
\phi\left(F_{1,1}^{1}\right)=2 l+r+r s+r t=2 l+2 r,
$$




$$
\begin{aligned}
& \phi\left(F_{1,2}^{1}\right)=o s \\
& \phi\left(F_{1,3}^{1}\right)=o t .
\end{aligned}
$$

We assume that the Bayesian update given an information set is a probability distribution over $H$ which gives probability 0 to all histories which are not an element of the information set. For an arbitrary prior belief $\phi=(l, l, r, r s, r t, o s, o t) \in \Phi_{(s, t)}^{0}$ the Bayesian update given $F_{1,1}^{1}$ is

$$
\operatorname{Bay}\left(\phi \mid F_{1,1}^{1}\right)=\left(\frac{l}{2 l+2 r}, \frac{l}{2 l+2 r}, \frac{r}{2 l+2 r}, \frac{r s}{2 l+2 r}, \frac{r t}{2 l+2 r}, 0,0\right) .
$$

Hence, the prior by prior Bayesian updates of $\Phi_{(s, t)}^{0}$ given the information sets $F_{1,1}^{1}, F_{1,2}^{1}$ and $F_{1,3}^{1}$ are

$$
\begin{aligned}
\operatorname{Bay}\left(\Phi_{(s, t)}^{0} \mid F_{1,1}^{1}\right) & =\left\{\left(\frac{l}{2 l+2 r}, \frac{l}{2 l+2 r}, \frac{r}{2 l+2 r}, \frac{r s}{2 l+2 r}, \frac{r t}{2 l+2 r}, 0,0\right) \text { s.t. }(l, r, o) \in \mathcal{P}\right\}, \\
& =\left\{(\tilde{l}, \tilde{l}, \tilde{r}, \tilde{r} s, \tilde{r} t, 0,0) \text { s.t. } \tilde{l} \in\left[0, \frac{\epsilon}{2}\right], \tilde{r} \in\left[\frac{1-\epsilon}{2}, \frac{1}{2}\right], 2 \tilde{l}+\tilde{r}+s \tilde{r}+t \tilde{r}=1\right\}, \\
& =\left\{(\tilde{l}, \tilde{l}, \tilde{r}, \tilde{r} s, \tilde{r} t, 0,0) \text { s.t. } \tilde{l} \in\left[0, \frac{\epsilon}{2}\right], \tilde{r} \in\left[\frac{1-\epsilon}{2}, \frac{1}{2}\right], \tilde{l}+\tilde{r}=\frac{1}{2}\right\}, \\
\operatorname{Bay}\left(\Phi_{(s, t)}^{0} \mid F_{1,2}^{1}\right) & =\{(0,0,0,0,0,1,0)\} \\
\operatorname{Bay}\left(\Phi_{(s, t)}^{0} \mid F_{1,3}^{1}\right) & =\{(0,0,0,0,0,0,1)\} .
\end{aligned}
$$

The rectangular hull rect $\left(\Phi_{(s, t)}^{0}\right)$ consist of all possible combination of marginal and updated beliefs. For the histories of the information sets $F_{1,2}^{1}$ and $F_{1,3}^{1}$ the updated belief is either 0 or 1 and since they consist only of one history, the prior probability of this history equals the marginal probability of reaching the information set. Multiplied with updated probability which is 1 is still the prior belief. Therefore, we focus on the information set $F_{1,1}^{1}$. Since $\tilde{l}$, $\tilde{r}, l$ and $r$ are elements of closed intervals we can focus on the all possible combination of the lowest and highest possible values for $\tilde{l}, \tilde{r}, l$ and $r$. Then the convex hull of the pasting of these distributions forms the rectangular hull.

Let $\phi$ be such that $r=1$ and $l=0$. Given this prior belief the marginal probability of reaching $F_{1,1}^{1}$ is $\phi\left(F_{1,1}^{1}\right)=2$. Let $\phi^{\prime}$ denote the pasting of the marginal $\phi\left(F_{1,1}^{1}\right)$ and the update $\tilde{\phi}$. Considering lowest and highest values for $\tilde{r}$ and $\tilde{l}$ there are two updated beliefs $\tilde{\phi}$ that can be pasted with this marginal belief:

- $\tilde{\phi}$ such that $\tilde{l}=0=\frac{1}{2}-\tilde{r}$

The pasting is then given by

$$
\begin{aligned}
\phi^{\prime} & =\phi \circ \tilde{\phi}=\left(\phi\left(F_{1,1}^{1}\right) \tilde{l}, \phi\left(F_{1,1}^{1}\right) \tilde{l}, \phi\left(F_{1,1}^{1}\right) \tilde{r}, \phi\left(F_{1,1}^{1}\right) \tilde{r} s, \phi\left(F_{1,1}^{1}\right) \tilde{r} t, 0,0\right) \\
& =\left(2 \cdot 0,2 \cdot 0,2 \cdot \frac{1}{2}, 2 \cdot \frac{1}{2} s, 2 \cdot \frac{1}{2} t, 0,0\right)
\end{aligned}
$$




$$
=(0,0,1, s, t, 0,0)
$$

- $\tilde{\phi}$ such that $\tilde{l}=\frac{\epsilon}{2}=\frac{1}{2}-\tilde{r}$

The pasting is then given by

$$
\begin{aligned}
\phi^{\prime} & =\phi \circ \tilde{\phi}=\left(\phi\left(F_{1,1}^{1}\right) \tilde{l}, \phi\left(F_{1,1}^{1}\right) \tilde{l}, \phi\left(F_{1,1}^{1}\right) \tilde{r}, \phi\left(F_{1,1}^{1}\right) \tilde{r} s, \phi\left(F_{1,1}^{1}\right) \tilde{r} t, 0,0\right) \\
& =\left(2 \cdot \frac{\epsilon}{2}, 2 \cdot \frac{\epsilon}{2}, 2 \cdot\left(\frac{1}{2}-\frac{\epsilon}{2}\right), 2 \cdot\left(\frac{1}{2}-\frac{\epsilon}{2}\right) s, 2 \cdot\left(\frac{1}{2}-\frac{\epsilon}{2}\right) t, 0,0\right) \\
& =(\epsilon, \epsilon, 1-\epsilon,(1-\epsilon) s,(1-\epsilon) t, 0,0) .
\end{aligned}
$$

Combining any possible combination of $(l, r, o)$ and $(\tilde{l}, \tilde{r})$ in such a way leads to the pastings given in Table 1. The probability of OS and OT follows from the pasting with the updated

\begin{tabular}{|c|c|c|}
\hline marginal & update & pasting \\
\hline$r=1, l=0$ & $\tilde{l}=0=\frac{1}{2}-\tilde{r}$ & $(0,0,1, s, t, 0,0)$ \\
$r=1, l=0$ & $\tilde{l}=\frac{\epsilon}{2}=\frac{1}{2}-\tilde{r}$ & $(\epsilon, \epsilon, 1-\epsilon,(1-\epsilon) s,(1-\epsilon) t, 0,0)$ \\
$r=1-\epsilon, l=\epsilon$ & $\tilde{l}=0=\frac{1}{2}-\tilde{r}$ & $(0,0,1, s, t, 0,0)$ \\
$r=1-\epsilon, l=\epsilon$ & $\tilde{l}=\frac{\epsilon}{2}=\frac{1}{2}-\tilde{r}$ & $(\epsilon, \epsilon, 1-\epsilon,(1-\epsilon) s,(1-\epsilon) t, 0,0)$ \\
$r=1-\epsilon, l=0$ & $\tilde{l}=0=\frac{1}{2}-\tilde{r}$ & $(0,0,1-\epsilon,(1-\epsilon) s,(1-\epsilon) t, \epsilon s, \epsilon t)$ \\
$r=1-\epsilon, l=0$ & $\tilde{l}=\frac{\epsilon}{2}=\frac{1}{2}-\tilde{r}$ & $\left((1-\epsilon) \epsilon,(1-\epsilon) \epsilon,(1-\epsilon)^{2},(1-\epsilon)^{2} s,(1-\epsilon)^{2} t, \epsilon s, \epsilon t\right)$ \\
\hline
\end{tabular}

Table 1: pasting for rectangular hull of player 1

belief given the information sets $F_{1,2}^{1}$ and $F_{1,3}^{1}$. For the first 4 rows the marginal probability of reaching $F_{1,2}^{1}$ or $F_{1,3}^{1}$ is 0 since $o=1-r-l=0$. For the last two rows $l=0$ and $r=1-\epsilon$ implies $o=\epsilon$. Since the prior by prior Bayesian update given $F_{1,2}^{1}$ or $F_{1,3}^{1}$ consist of just one belief, which gives probability 1 to $O S$ or OT, respectively, the pasting of marginal and update for $O S$ and $O T$ equals the values given above.

The rectangular hull $\operatorname{rect}\left(\Phi_{(s, t)}^{0}\right)$ of player 1 is then given by the convex hull of the pastings given in Table 1

$$
\begin{array}{r}
\operatorname{rect}\left(\Phi_{(s, t)}^{0}\right)=\operatorname{conv}\{(0,0,1-\epsilon,(1-\epsilon) s,(1-\epsilon) r, \epsilon s, \epsilon r),(\epsilon, \epsilon, 1-\epsilon,(1-\epsilon) s,(1-\epsilon) r, 0,0), \\
\left.(0,0,1, s, t, 0,0),\left((1-\epsilon) \epsilon,(1-\epsilon) \epsilon,(1-\epsilon)^{2},(1-\epsilon)^{2} s,(1-\epsilon)^{2} t, \epsilon s, \epsilon t\right)\right\} .
\end{array}
$$

To see the difference between the rectangular hull and $\Phi_{(s, t)}^{0}$, remember that $\Phi_{(s, t)}^{0}$ is given by

$$
\begin{aligned}
\Phi_{(s, t)}^{0} & =\{(l, l, r, r s, r t, o s, o t) \text { s.t. }(l, r, o) \in \mathcal{P}\} \\
& =\operatorname{conv}\{(0,0,1-\epsilon,(1-\epsilon) s,(1-\epsilon) r, \epsilon s, \epsilon r),(\epsilon, \epsilon, 1-\epsilon,(1-\epsilon) s,(1-\epsilon) r, 0,0), \\
& (0,0,1, s, t, 0,0)\} .
\end{aligned}
$$


Since the belief $\left((1-\epsilon) \epsilon,(1-\epsilon) \epsilon,(1-\epsilon)^{2},(1-\epsilon)^{2} s,(1-\epsilon)^{2} t, \epsilon s, \epsilon t\right)$ is not an element of $\Phi_{(s, t)}^{0}$ it follows that $\Phi_{(s, t)}^{0} \subsetneq \operatorname{rect}\left(\Phi_{(s, t)}^{0}\right)$. The last row in Table 1 shows that this belief is constructed by pasting the marginal probability of the prior worst case belief with the interim worst case belief. We will see later, that this belief change the ex ante optimal behavior such that player 1 plays dynamically consistently.

Similar calculations as above show that the Bayesian update of $\Phi_{(m, n)}^{0}$ given $F_{2,1}^{1}$ and the rectangular hull $\operatorname{rect}\left(\Phi_{(m, n)}^{0}\right)$ of player 2 are given by

$$
\begin{array}{r}
\left.\operatorname{Bay}\left(\Phi_{(m, n)}^{0} \mid F_{2,1}^{1}\right)\right)=\left\{(0,0,0, \tilde{r n}, \tilde{r n}, \tilde{o}, \tilde{o}) \text { with } \tilde{r n} \in\left[\frac{(1-\epsilon) n}{2(1-\epsilon) n+2 \epsilon}, \frac{1}{2}\right]\right. \\
\left.\tilde{o} \in\left[0, \frac{\epsilon}{2 n(1-\epsilon)+2 \epsilon}\right], \tilde{o}+\tilde{r n}=\frac{1}{2}\right\}
\end{array}
$$

and

$$
\begin{aligned}
\operatorname{rect}\left(\Phi_{(m, n)}^{0}\right)= & \operatorname{conv}\{(0,0,(1-\epsilon) m,(1-\epsilon) n+\epsilon,(1-\epsilon) n+\epsilon, 0,0) \\
& \left(0,0, m, \frac{(1-\epsilon) n^{2}}{(1-\epsilon) n+\epsilon}, \frac{(1-\epsilon) n^{2}}{(1-\epsilon) n+\epsilon}, \frac{\epsilon n}{(1-\epsilon) n+\epsilon}, \frac{\epsilon n}{(1-\epsilon) n+\epsilon}\right), \\
& (0,0,(1-\epsilon) m,(1-\epsilon) n,(1-\epsilon) n, \epsilon, \epsilon),(0,0, m, n, n, 0,0) \\
& (\epsilon m, \epsilon n,(1-\epsilon) m,(1-\epsilon) n,(1-\epsilon) n, 0,0), \\
& \left.\left(\epsilon m, \epsilon n,(1-\epsilon) m, \frac{(1-\epsilon)^{2} n^{2}}{(1-\epsilon) n+\epsilon}, \frac{(1-\epsilon)^{2} n^{2}}{(1-\epsilon) n+\epsilon}, \frac{(1-\epsilon) \epsilon n}{(1-\epsilon) n+\epsilon}, \frac{(1-\epsilon) \epsilon n}{(1-\epsilon) n+\epsilon}\right)\right\} .
\end{aligned}
$$

The rectangular belief set of player 1 shows the main differences of $\Phi_{\sigma_{-i}}$ and $\operatorname{rect}\left(\Phi_{\sigma_{-i}}\right)$. The rectangular hull contains the belief which is the pasting of the prior and interim worst case belief. We will see later that due to rectangularity the prior worst case belief given the rectangular hull will be the pasting of the marginal belief derived from the prior worst case belief and the interim worst case belief. Therefore, updating leads to the interim worst case belief and dynamically inconsistent behavior cannot occur.

Given the complex structure of beliefs described in the last section one might wonder why we are not proceeding by constructing rectangular prior belief sets over the set of types $H^{0}$ and updating these sets prior by prior using Bayes' rule. As Aryal and Stauber (2014) and Ellis (2018) show, defining common belief sets over the set of types $H^{0}$ that are rectangular for all players is not possible for general games. For our analysis it is essential that the information structure of the game is given by a sequence of partitions of a fixed set. Updating beliefs on $H^{0}$ given an information set $I_{i}^{t}$ leads to updated beliefs as probability distributions over the partial histories up to stage $t$, i.e. the set of which information sets form the partition is changing every period. Furthermore, there can exist paths that are starting from the same type or state but are leading to different information sets depending on the action of the opponent. In our running example the histories $R M$, $R N S$ and $R N T$ lead to different information sets of player 2. First, this shows that it is 
not possible to define a partition of $\{L, R, O\}$ that captures the information player 2 gets at his information set at the interim stage. Furthermore, which information set is reached depends on the action of player 1 . When constructing rectangular beliefs for all players, we have to consider this dependence of information sets and actions of opponents. ${ }^{12}$ The rectangular hull of player 2 shows this dependence. The beliefs depend on the strategy of the opponent in such a way, that an arbitrary belief $\phi \in \operatorname{rect}\left(\Phi_{\sigma_{-i}}^{0}\right)$ cannot be represented by multiplying the transition probability $p_{\sigma_{-i}}\left(h \mid h^{0}\right)$ with imprecise probabilistic information $\pi \in \mathcal{P}$. Furthermore, in Example 3 in the Appendix we show that $\operatorname{rect}\left(\Phi_{\sigma_{-i}}\right)$ for different $\sigma_{-i}$ not only differs because of different transition probabilities $p_{\sigma_{-i}}(\cdot \mid \cdot)$. Therefore, we cannot construct rectangular prior belief sets over $H^{0}$.

\section{$3 \quad$ Ex-Ante and Interim Equilibria}

In this section we define ex-ante and interim expected utility and equilibria. We assume that given the ex-ante or interim set of beliefs players evaluate a strategy profile by choosing the worst case belief and calculating the expected payoff for this belief.

Definition 7 The ex-ante expected utility for agent $i$ of a strategy profile $\sigma=\left(\sigma_{i}, \sigma_{-i}\right)$ is given by

$$
U^{e}\left(\left(\sigma_{i}, \sigma_{-i}\right)\right)=\min _{\phi \in \Psi_{i}^{0}} \sum_{h \in H} u_{i}(h) p_{\sigma_{i}}\left(h \mid h^{0}\right) \phi(h) .
$$

Similarly the interim expected utility at $F_{i}^{t} \in \mathcal{F}_{i}^{t}$ at stage $t=0, \ldots, T$ given a belief set $\Psi_{i}^{t}\left(F_{i}^{t}\right)$ is

$$
U_{i}^{i}\left(\sigma, \Psi_{i}^{t}\left(F_{i}^{t}\right)\right)=\min _{\phi \in \Psi_{i}^{t}\left(F_{i}^{t}\right)} \sum_{h \in H} u_{i}(h) p_{\sigma_{i}}\left(h \mid h^{t}\right) \phi(h) .
$$

Given the ex-ante and interim expected utility of the agents the definitions of an ex-ante and interim equilibrium are straightforward and follow the standard idea of Nash Equilibrium.

Definition 8 A strategy profile $\sigma^{*}$ is an ex-ante equilibrium with rectangular beliefs if and only if $\Psi_{i}^{0}=\operatorname{rect}\left(\Phi_{\sigma_{-i}^{*}}^{0}\right)$ and

$$
U_{i}^{e}\left(\sigma^{*}\right) \geq U^{e}\left(\sigma_{i}^{\prime}, \sigma_{-i}^{*}\right)
$$

for all $\sigma_{i}^{\prime} \in \Sigma_{i}$ and $i \in N$.

\footnotetext{
${ }^{12}$ Aryal and Stauber (2014) construct a rectangular belief sets of beliefs over $\{L, R, O\}$ of player 1 in our running example. Then they transfer this belief set to a state space that consider the difference between the partial histories $R N$ and $R M$ and shows that this transferred belief set is not rectangular for player 2 . We are proceeding the other way around. We first transfer the set of imprecise probabilistic information to heterogeneous beliefs sets on $H$ and then construct the rectangular hull for each player.
} 
Definition 9 A tuple $\left(\sigma^{*}, \Psi^{t}\right)$ consisting of a strategy profile $\sigma^{*}$ and system of beliefs $\Psi^{t}$ at $t$ is an interim equilibrium with rectangular beliefs at stage $t$ if and only if $\Psi_{i}^{t}\left(F_{i}^{t}\right)=\operatorname{rect}\left(\Phi_{\sigma_{-i}^{*}}^{t}\left(F_{i}^{t}\right)\right)$ for all $F_{i}^{t} \in \mathcal{F}_{i}^{t}$ and

$$
U_{i}^{i}\left(\sigma^{*}, \Psi_{i}^{t}\left(F_{i}^{t}\right)\right) \geq U_{i}^{i}\left(\left(\sigma_{i}^{\prime}, \sigma_{-i}^{*}\right), \Psi_{i}^{t}\left(F_{i}^{t}\right)\right)
$$

for all $\sigma_{i}^{\prime} \in \Sigma_{i}, F_{i}^{t} \in \mathcal{F}_{i}^{t}$ and all $i \in N$.

Without assuming rectangularity existence of an ex-ante equilibrium follows from standard arguments using that $\phi(h)=p_{\sigma_{-i}}\left(h \mid h^{0}\right) \pi\left(h^{0}\right)$ for all $\phi \in \Phi_{\sigma_{-i}}^{0}$. With rectangularity this simple characterization of the beliefs does not hold in general. Therefore, we focus on the proof of existence of equilibria with rectangular beliefs.

Theorem 1 (Existence ex-ante equilibrium with rectangular beliefs) There exists an ex-ante equilibrium with rectangular beliefs.

The proof of Theorem 1 follows the usual idea using Kakutani's fixed point theorem and can be found in the Appendix. To use Kakutani's fixed point theorem it is essentially that $U_{i}^{e}\left(\left(\sigma_{i}, \sigma_{-i}\right)\right)$ is jointly continuous in $\left(\sigma_{i}, \sigma_{-i}\right)$. Due to our definition of beliefs, the strategy of the opponents influence the set of beliefs over which a player minimize. Therefore the continuity is not trivial and we need the following Lemma for the proof of Theorem 1.

Lemma $1 U_{i}^{e}\left(\left(\sigma_{i}, \sigma_{-i}\right)\right)$ with $\Psi_{i}^{0}=\operatorname{rect}\left(\Phi_{\sigma_{-i}}^{0}\right)$ is jointly continuous in $\left(\sigma_{i}, \sigma_{-i}\right)$.

To prove continuity we use that any belief in $\operatorname{rect}\left(\Phi_{\sigma_{-i}}^{0}\right)$ can be represented by the pasting of marginal and updated belief. Due to Remark 2 marginal and updated beliefs can be represented by multiplying $p_{\sigma_{-i}}(h \mid \cdot)$ and $\pi \in \mathcal{P}$. This leads to the following representation of an arbitrary element $\phi \in \operatorname{rect}\left(\Phi_{\sigma_{-i}}^{0}\right)$.

$$
\phi(h)=\sum_{F_{i}^{1} \in \mathcal{F}_{i}^{1}}\left(\sum_{h \in F_{i}^{1}} p_{\sigma_{-i}}\left(h \mid h^{0}\right) \pi\left(h^{0}\right)\right) \frac{p_{\sigma_{-i}}\left(h \mid h^{0}\right) \pi_{F_{i}^{1}}^{\prime}\left(h^{0}\right)}{\sum_{h \in F_{i}^{1}} p_{\sigma_{-i}}\left(h \mid h^{0}\right) \pi_{F_{i}^{1}}^{\prime}\left(h^{0}\right)} .
$$

Using this representation we transform $U_{i}^{e}\left(\left(\sigma_{i}, \sigma_{-i}\right)\right)$ to a minimization problem over the set $\left(\pi,\left(\pi^{\prime}\right)_{F_{i}^{1}}\right) \in \mathcal{P} \times \mathcal{P}^{\left|\mathcal{F}_{i}^{1}\right|}$ which is independent of $\sigma_{-i}$. Then, jointly continuity of the transformed problem follows by standard methods.

Theorem 2 (Existence interim equilibrium with rectangular beliefs) There exists an interim equilibrium with rectangular beliefs at stage $t$.

Proof. The compactness of $\mathcal{P}$ implies compactness of $\operatorname{Bay}\left(\mathcal{P} \mid F_{i}^{t}\right)$ for any $F_{i}^{t}$. Hence, replacing $\operatorname{rect}\left(\Phi_{\sigma_{-i}}^{0}\right)$ in Lemma 1 by $\operatorname{rect}\left(\Phi_{\sigma_{-i}}^{t}\left(F_{i}^{t}\right)\right)$ shows jointly continuity of $U_{i}^{i}$. Then the proof follows the same line as the proof of Theorem 1. 


\subsection{Relation of Ex-Ante and Interim Equilibrium}

Now we come back to the problem of dynamic consistency and show that stability under pasting induce dynamically consistent behavior.

Our next theorem shows that under stability under pasting a completely mixed exante equilibrium implies an interim equilibrium with rectangular beliefs. Therefore, as in games without ambiguity a player would deviate from an ex-ante optimal strategy only at out-of-equilibrium information sets.

Theorem 3 (ex-ante implies interim) Let $\sigma^{*}$ be a completely mixed ex-ante equilibrium with rectangular beliefs. Then $\left(\sigma^{*}, \Psi_{i}^{t}\right)$ with $\Psi_{i}^{t}\left(F_{i}^{t}\right)=\operatorname{rect}\left(\Phi_{\sigma_{-i}^{*}}^{t}\left(F_{i}^{t}\right)\right)$ is an interim equilibrium with rectangular beliefs at stage $t$.

We will prove this theorem by showing that due to stability under pasting a completely mixed interim equilibrium at $t-1$ implies an completely mixed equilibrium at $t$. This holds for any arbitrary $t=0, \ldots T+1$ and Theorem 3 follows by iteration. The following Corollary follows immediately from the recursive structure of the proof

Corollary 1 Let $\left(\sigma^{*}, \Psi_{i}^{t-1}\right)$ be a completely mixed interim equilibrium at $t-1$ with rectangular beliefs, i.e. $\Psi_{i}^{t-1}\left(F_{i}^{t-1}\right)=\operatorname{rect}\left(\Phi_{\sigma_{-i}^{*}}^{t-1}\left(F_{i}^{t-1}\right)\right)$. Then $\left(\sigma^{*}, \Psi_{i}^{t}\right)$ with $\Psi_{i}^{t}\left(F_{i}^{t}\right)=\operatorname{rect}\left(\Phi_{\sigma_{-i}^{*}}^{t}\left(F_{i}^{t}\right)\right)$ is an interim equilibrium with rectangular beliefs at stage $t$.

The formal proof of Theorem 3 may be found in the Appendix. To give an intuition for the result we need the following Lemma. It shows the relation between the worst case expected utility at different stages and is essential for the relation between interim and ex ante equilibrium.

Lemma 2 Let $\phi^{*} \in \operatorname{argmin}_{\phi \in \operatorname{rect}\left(\Phi_{\sigma_{-i}}^{t-1}\left(F_{i}^{t-1}\right)\right)} \sum_{h \in H} u_{i}(h) p_{\sigma_{i}}\left(h \mid h^{t-1}\right) \phi(h)$. Then

$$
\begin{aligned}
\sum_{h \in F_{i}^{t-1}} u_{i}(h) p_{\sigma_{i}} & \left(h \mid h^{t-1}\right) \phi^{*}(h) \\
& =\sum_{F_{i}^{t} \in \mathcal{F}_{i}^{t}} \phi^{*}\left(F_{i}^{t}\right) p_{\sigma_{i}}\left(h^{t} \mid h^{t-1}\right) \sum_{\tilde{\phi} \in \operatorname{rect}\left(\Phi_{\sigma_{-i}}^{t}\left(F_{i}^{t}\right)\right)} \sum_{h \in H} u_{i}(h) p_{\sigma_{i}}\left(h \mid h^{t}\right) \tilde{\phi}(h) .
\end{aligned}
$$

We give a sketch of the proof since it helps to understand the role of rectangularity. The proof consists of two steps. First, Bayesian updating implies that the left hand side of Equation 1 is greater or equal than the right hand side. The other direction follows from rectangularity. Due to rectangularity there exist a $\phi^{\prime} \in \Phi_{\sigma_{-i}}^{t-1}\left(F_{i}^{t-1}\right)$ such that

$$
\phi^{\prime}(h)=\sum_{F_{i}^{t} \in \mathcal{F}_{i}^{t}} \phi^{*}\left(F_{i}^{t}\right) \tilde{\phi}_{F_{i}^{t}}^{*}(h)
$$

where $\tilde{\phi}_{F_{i}^{t}}^{*}$ and $\phi^{*}$ are the worst case beliefs at $F_{i}^{t}$ or $F_{i}^{t-1}$, respectively, i.e. $\phi^{\prime}$ is the pasting of the worst case beliefs at $t$ and $t-1$. We still do not know if $\phi^{\prime}$ is the worst case belief 
at $t-1$. Therefore, the left hand side of Equation 1 can be smaller or equal than the left hand side evaluated with the belief $\phi^{\prime}$ instead of $\phi^{*}$. Then, using that $\phi^{\prime}$ is the pasting of the worst case beliefs $\phi^{*}$ and $\tilde{\phi}_{F_{i}^{t}}^{*}$ we can prove that the left hand side of Equation 1 evaluated with $\phi^{\prime}$ equals the right hand side of Equation 1.

As the next remark shows, Lemma 2 implies that the worst case belief at $F_{i}^{t-1}$ is the pasting of the worst case belief at $F_{i}^{t-1}$ and the worst case beliefs at $t$. Therefore, the Bayesian update of the worst case belief at $F_{i}^{t-1}$ leads to the worst case belief at all subsequent information sets at $t$.

Remark 4 From the proof of Lemma 2 we know that there exist a $\phi^{\prime} \in \Phi_{\sigma_{-i}}^{t-1}\left(F_{i}^{t-1}\right)$ which satisfies Equation 2, i.e.

$$
\phi^{\prime}(h)=\sum_{F_{i}^{t} \in \mathcal{F}_{i}^{t}} \phi^{*}\left(F_{i}^{t}\right) \tilde{\phi}_{F_{i}^{t}}^{*}(h)
$$

where $\phi^{*}$ and $\tilde{\phi}_{F_{i}^{t}}^{*}$ are the worst case beliefs at $F_{i}^{t-1}$ and $F_{i}^{t}$, respectively. Furthermore, the proof states

$$
\begin{aligned}
\sum_{h \in F_{i}^{t-1}} u_{i}(h) p_{\sigma_{i}}\left(h \mid h^{t-1}\right) \phi^{*}(h) & \leq \sum_{h \in F_{i}^{t-1}} u_{i}(h) p_{\sigma_{i}}\left(h \mid h^{t-1}\right) \phi^{\prime}(h) \\
& =\sum_{F_{i}^{t} \in \mathcal{F}_{i}^{t}} \phi^{*}\left(F_{i}^{t}\right) p_{\sigma_{i}}\left(h^{t} \mid h^{t-1}\right) \min _{\tilde{\phi} \in \Phi_{\sigma_{-i}}^{t}\left(F_{i}^{t}\right)} \sum_{h \in F_{i}^{t}} u_{i}(h) p_{\sigma_{i}}\left(h \mid h^{t}\right) \tilde{\phi}(h) .
\end{aligned}
$$

But by Lemma 2 we know that the inequality is an equality. Therefore, $\phi^{\prime}$ is a worst case belief at $F_{i}^{t-1}$ and Remark 2 implies that the worst case belief at an information set at stage $t$ is the Bayesian update of the worst case belief of the previous information set at stage $t-1$.

Remark 4 and Lemma 2 show how rectangularity leads to dynamically consistent behavior which is necessary for the proof of Theorem 3.

The proof of Theorem 3 follows the usual idea of contraposition. If there would exist a profitable deviation at $t$ this deviation would be profitable at $t-1$ as well. Therefore, an equilibrium at $t-1$ implies an equilibrium at $t$. Then the theorem follows from iteration. However, one has to consider the worst case beliefs. The belief set only depends on the strategy of the opponents. Therefore, fixing the strategy of the opponents leads to fixed belief sets. But the worst case belief of player $i$ may change if he deviates from the equilibrium strategy. To prove Theorem 3 we have to define the pasting of the worst case belief at $t$ given the equilibrium strategy with the worst case belief at $t-1$ given the deviation strategy. Due to rectangulatrity this pasting is an element of the belief set at $t-1$. Then, we use Lemma 2 to show that a profitable deviation at $t$ implies a profitable deviation at $t-1$.

Theorem 3 shows the relation between equilibria at different stages. Roughly speaking, due to stability under pasting players update their beliefs such that their worst case belief 
at $t$ is the Bayesian update of the worst case belief at $t-1$. This implies dynamically consistent behavior and leads to the relation between ex-ante and interim equilibria stated in Theorem 3.

Now we come back to our running example and show that dynamic inconsistency is ruled out due to stability under pasting.

Running Example (cont.) Remember the results from above. Without stability under pasting, player 1 behaves dynamically inconsistently. His optimal ex-ante strategy is to play $M$ with probability 1. After learning that the state is not $O$ his optimal interim strategy is to play $M$ with probability $\frac{1}{102}$ if $\epsilon>\frac{1}{102}$. Now we will show that beliefs which are stable under pasting lead to dynamically consistent behavior. Since player 2 is indifferent between $S$ and $T$ we still focus on player 1 . We already know the information partitions and rectangular beliefs of player 1 .

$$
\begin{aligned}
& H=\{L M, L N, R M, R N S, R N T, O S, O T\} \\
& \mathcal{F}_{i}^{0}= H \\
& \mathcal{F}_{1}^{1}=\left\{F_{1,1}^{1}, F_{1,2}^{1}\right\}=\{\{L M, L N, R M, R N S, R N T\},\{O S, O T\}\} \\
& \Psi_{1}^{0}=\operatorname{rect}\left(\Phi_{(s, t)}^{0}\right)=\operatorname{conv}\{(0,0,1-\epsilon,(1-\epsilon) s,(1-\epsilon) r, \epsilon s, \epsilon r),(0,0,1, s, t, 0,0), \\
&(\epsilon, \epsilon, 1-\epsilon,(1-\epsilon) s,(1-\epsilon) r, 0,0), \\
&\left.\left((1-\epsilon) \epsilon,(1-\epsilon) \epsilon,(1-\epsilon)^{2},(1-\epsilon)^{2} s,(1-\epsilon)^{2} t, \epsilon s, \epsilon t\right)\right\} .
\end{aligned}
$$

From Remark 2 we know that the update of $\operatorname{rect}\left(\Phi_{(s, t)}^{0}\right)$ is the same as the update of $\Phi_{(s, t)}^{0}$. Therefore, the optimal interim strategy with rectangular beliefs is the same as without stability under pasting, i.e. $m^{*}=\frac{1}{102}$ if $\epsilon>\frac{1}{102}$. For the optimal ex-ante strategy with rectangular beliefs we solve the following problem

$$
\begin{aligned}
& \max _{(1-m, m)} U_{1}^{e}((1-m, m))=\max _{(1-m, m)} \min _{\phi \in \operatorname{rect}\left(\Phi_{(s, t)}^{0}\right)} 101(1-m) \phi(L N)+101 m \phi(R M) \\
& +100(1-m) \phi(R N T)+100(1-m) \phi(R N S)-\phi(O S)-\phi(O T) \\
& =\max _{(1-m, m)} \min _{\substack{l, l, r, r s, r t, o s, o t) \\
\in \operatorname{rect}\left(\Phi_{(s, t)}^{0}\right)}} 101(1-m) l+101 m r+100(1-m) r s+100(1-m) r(1-s)-o \\
& =\max _{(1-m, m)} \min _{\substack{l, l, l, r, r s, r t, o s, o t) \\
\in \operatorname{rect}\left(\Phi_{(s, t)}^{0}\right)}} 101(1-m) l+101 m r+100(1-m) r-(1-r-l) \\
& =\max _{(1-m, m)} \min _{\substack{l l, l, r, r s, r t, o s, o t) \\
\in \operatorname{rect}\left(\Phi_{(s, t)}^{0}\right)}} l(101(1-m)-1)+r(100+m-1)+1 .
\end{aligned}
$$

The worst case belief depends on $m$. If $101(1-m)>100+m$ the worst case belief gives the lowest possible value to $l$, the highest value to o and $r=1-l-o$. If $101(1-m) \leq 100+m$ the worst case belief gives the highest value to o, the lowest value to $r$ and $l=1-o-r$. 
Hence, the worst case belief is

$$
\phi^{*}= \begin{cases}(0,0,1-\epsilon,(1-\epsilon) s,(1-\epsilon) t, \epsilon s, \epsilon t) & \text { if } m<\frac{1}{102} \\ \left(\epsilon(1-\epsilon), \epsilon(1-\epsilon),(1-\epsilon)^{2},(1-\epsilon)^{2} s,(1-\epsilon)^{2} t, \epsilon s, \epsilon t\right) & \text { if } m \geq \frac{1}{102}\end{cases}
$$

The worst case ex-ante utility is

$$
\begin{aligned}
U_{1}^{e}((1-m, m)) & = \begin{cases}(1-\epsilon)(100+m-1)+1 & \text { if } m<\frac{1}{102} \\
(1-\epsilon) \epsilon(101(1-m)-1)+(1-\epsilon)^{2}(100+m-1)+1 & \text { if } m \geq \frac{1}{102}\end{cases} \\
& = \begin{cases}(1-\epsilon)(99+m)+1 & \text { if } m<\frac{1}{102} \\
m(1-\epsilon)(1-102 \epsilon)+(1-\epsilon)(99+\epsilon)+1 & \text { if } m \geq \frac{1}{102} .\end{cases}
\end{aligned}
$$

Hence, the optimal ex-ante strategy is $m^{*}=\frac{1}{102}$ if $\epsilon>\frac{1}{102}$ which proves dynamic consistency.

The example shows that players chooses their ex-ante belief in such a way that the belief set includes prior beliefs that allow for dynamically consistent behavior. The structure of the game and possible future information are additional information that influences the behavior of the agents. A rational agent take this information into account, when he forms his prior beliefs. Therefore, his prior beliefs not only depend on the imprecise probabilistic information $\mathcal{P}$ but also on the knowledge that player have about the structure of the game.

Given the relation between ex-ante and interim equilibria discussed in this section we can now define and prove the existence of Sequential Equilibria.

\section{Sequential Equilibrium}

Kreps and Wilson (1982) define a Sequential Eequilibrium in a game without ambiguity as a tuple of a strategy profile and a belief system such that the strategy profile is sequential rational and the belief system consistent with respect to the strategy profile. Consistent with respect to to a strategy profile $\sigma$ means that there exist a sequence of completely mixed strategy profiles that converges to $\sigma$ such that the sequence of beliefs constructed by Bayesian updating given the completely mixed strategy profiles converges to the equilibrium belief. We use a similar notion of consistent that includes stability under pasting.

Fix a sequence $\epsilon^{k}=\left(\epsilon_{I}^{k}\right)_{I \in \cup_{i \in N} \mathcal{I}_{i}}$ of strictly positive vectors converging in the sup-norm to 0 and such that $\epsilon_{I_{i}}^{k} \leq \frac{1}{\left|A_{i}\left(I_{i}\right)\right|}$ for all player $i$ and information sets $I_{i}$. For any k let $\Gamma^{k}$ denote the restriction of $\Gamma$ such that the set of feasible strategies is the set of all completely mixed $\sigma^{k}$ satisfying $\sigma_{i}^{k}\left(I_{i}\right)\left(a_{i}\right) \geq \epsilon_{I_{i}}^{k}$ for all players, information sets and actions $a_{i} \in A_{i}\left(I_{i}\right)$. Let $\Sigma^{k}$ denote the set of strategy profiles satisfying this constrain. For every strategy profile in $\Sigma^{k}$ Bayes' rule is always well defined. Let $\left(\sigma^{k}\right)_{k}$ converge to $\sigma \in \Sigma$ such that $\sigma^{k} \in \Sigma^{k}$. For each player $i$ and each $\sigma_{-i}^{k}$ we can construct a prior belief set $\operatorname{rect}\left(\Phi_{\sigma_{-i}^{k}}^{0}\right)$ which is stable under pasting. Now we construct a prior belief system given $\sigma$ which is stable under pasting 
and consistent with $\sigma_{-i}^{k}$. First note that

$$
\lim _{k \rightarrow \infty} p_{\sigma_{-i}^{k}}\left(h^{s} \mid h^{t}\right)=p_{\sigma_{-i}}\left(h^{s} \mid h^{t}\right) \quad \forall s, t=0 \ldots T, s \geq t .
$$

Take an arbitrary tuple $\left(\pi,\left(\pi_{F_{i}^{1}}^{\prime}\right)_{F_{i}^{1} \in \mathcal{F}_{i}^{1}}\right) \in \mathcal{P} \times \mathcal{P}^{\left|\mathcal{F}_{i}^{1}\right|}$. Then there exist a sequence of $\phi^{k} \in \operatorname{rect}\left(\Phi_{\sigma_{-i}^{k}}^{0}\right)$ such that

$$
\begin{aligned}
\phi^{k}(h) & =\sum_{F_{i}^{1} \in \mathcal{F}_{i}^{1}} \phi^{k}\left(F_{i}^{1}\right) \bar{\phi}_{F_{i}^{1}}^{k}(h) \\
& =\sum_{F_{i}^{1} \in \mathcal{F}_{i}^{1}}\left(\sum_{h \in F_{i}^{1}} p_{\sigma_{-i}^{k}}\left(h \mid h^{0}\right) \pi\left(h^{0}\right)\right) \frac{p_{\sigma_{-i}^{k}}\left(h \mid h^{0}\right) \pi_{F_{i}^{1}}^{\prime}\left(h^{0}\right)}{\sum_{h \in F_{i}^{1}} p_{\sigma_{-i}^{k}}\left(h \mid h^{0}\right) \pi_{F_{i}^{1}}^{\prime}\left(h^{0}\right)} .
\end{aligned}
$$

Then

$$
\lim _{k \rightarrow \infty} \phi^{k}(h)=\sum_{F_{i}^{1} \in \mathcal{F}_{i}^{1}}\left(\sum_{h \in F_{i}^{1}} p_{\sigma_{-i}}\left(h \mid h^{0}\right) \pi\left(h^{0}\right)\right) \lim _{k \rightarrow \infty} \frac{p_{\sigma_{-i}^{k}}\left(h \mid h^{0}\right) \pi_{F_{i}^{1}}^{\prime}\left(h^{0}\right)}{\sum_{h \in F_{i}^{1}} p_{\sigma_{-i}^{k}}\left(h \mid h^{0}\right) \pi_{F_{i}^{1}}^{\prime}\left(h^{0}\right)} .
$$

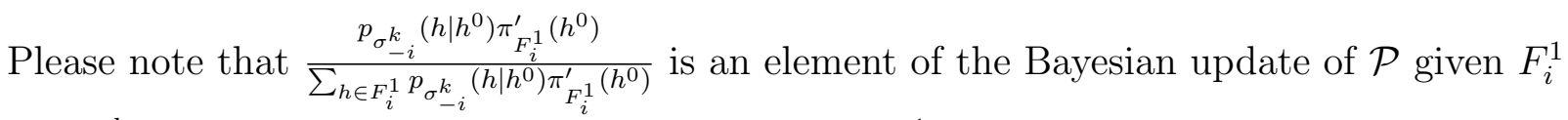
and $\sigma^{k}$ for each $k$ and therefore an element of $\Delta H^{1}$, the set of probability distributions over partial histories at stage 1. Furthermore, taking the limit of $\phi^{k}$ only influence the path probability induced by the strategy of the opponents, i.e. $\pi$ and $\pi_{F_{i}^{1}}^{\prime}$ are fixed. Then, the compactness of $\Delta H^{1}$ implies that every sequence of $\frac{p_{\sigma_{-i}^{k}}\left(h \mid h^{0}\right) \pi_{F_{i}^{1}}^{\prime}\left(h^{0}\right)}{\sum_{h \in F_{i}^{1}} p_{\sigma_{-i}^{k}}\left(h \mid h^{0}\right) \pi_{F_{i}^{1}}^{\prime}\left(h^{0}\right)}$ has a convergent subsequence.

The limit of $\operatorname{rect}\left(\Phi_{\sigma_{-i}^{k}}\right)$ is then defined as

$$
\begin{array}{r}
\lim _{k \rightarrow \infty} \operatorname{rect}\left(\Phi_{\sigma_{-i}^{k}}^{0}\right):=\left\{\phi \in[0,1]^{H} \text { such that } \exists\left(\phi^{k}\right)_{k=1,2, \ldots} \in\left(\operatorname{rect}\left(\Phi_{\sigma_{-i}^{k}}^{0}\right)\right)_{k=1,2, \ldots}\right. \text { with } \\
\left.\phi(h)=\lim _{k \rightarrow \infty} \phi^{k}(h)\right\} .
\end{array}
$$

Similarly one can define the limit of rectangular interim belief sets at stages $t>0$.

By construction $\lim _{k} \operatorname{rect}\left(\Phi_{\sigma_{-i}^{k}}^{t-1}\left(F_{i}^{t-1}\right)\right)$ is stable under pasting as the following calculations show. Let $\phi \in \lim _{k} \operatorname{rect}\left(\Phi_{\sigma_{-i}^{t}}^{t-1}\left(F_{i}^{t-1}\right)\right)$ and $\left(\phi_{F_{i}^{t}}\right)_{F_{i}^{t}} \in\left(\operatorname{Bay}\left(\lim _{k} \operatorname{rect}\left(\Phi_{\sigma_{-i}^{k}}^{t-1}\left(F_{i}^{t-1}\right)\right) \mid F_{i}^{t}\right)\right)_{F_{i}^{t}}$. We have to show that the pasting of $\phi$ and $\left(\phi_{F_{i}^{t}}\right)_{F_{i}^{t}}$ is an element of $\lim _{k} \operatorname{rect}\left(\Phi_{\sigma_{-i}^{k}}^{t-1}\left(F_{i}^{t-1}\right)\right)$.

$$
\phi \circ\left(\phi_{F_{i}^{t}}\right)_{F_{i}^{t}}(\cdot)=\sum_{F_{i}^{t} \in \mathcal{F}_{i}^{t}} \lim _{k} \phi^{k}\left(F_{i}^{t}\right) \lim _{k} \phi_{F_{i}^{t}}(\cdot)
$$




$$
\begin{aligned}
& =\lim _{k} \underbrace{\sum_{F_{i}^{t} \in \mathcal{F}_{i}^{t}} \phi^{k}\left(F_{i}^{t}\right) \phi_{F_{i}^{t}}(\cdot)}_{:=\bar{\phi}^{k} \in \operatorname{rect}\left(\Phi_{\sigma_{-i}^{t}}^{t-1}\right)} \\
& =\lim _{k} \bar{\phi}^{k}(\cdot) \in \lim _{k} \operatorname{rect}\left(\Phi_{\sigma_{-i}^{k}}^{t-1}\left(F_{i}^{t-1}\right)\right) .
\end{aligned}
$$

This shows that rectangularity is maintained under the limit. Now we can define consistent with respect to a strategy profile $\sigma$ and sequential rationality for rectangular beliefs.

Definition 10 We say a belief system $\Psi$ is consistent w.r.t. $\sigma$ if there exist a sequence $\left(\sigma^{k}\right)_{k=1, \ldots}$ such that

- $\sigma^{k} \in \Sigma^{k}$ for all $k$,

- $\sigma=\lim _{k} \sigma^{k}$,

- $\Psi_{i}^{t}\left(F_{i}^{t}\right)=\left\{\phi: \phi(h)=\lim _{k} \phi^{k}(h)\right.$ where $\left.\phi^{k} \in \operatorname{Bay}\left(\operatorname{rect}\left(\Phi_{\sigma_{-i}^{k}}^{0} \mid F_{i}^{t}\right)\right)\right\}$ for all $F_{i}^{t} \in \mathcal{F}_{i}^{t}$ for all $t>0$.

The definition of consistent and the discussion above show that a belief system which is consistent w.r.t. $\sigma$ and stable under pasting for $\sigma^{k}$ is stable under pasting for $\sigma$.

The second property of Sequential Equilibria is sequential rationality. Roughly speaking, sequential rationality captures the idea that a strategy is optimal at each stage and each information set. Therefore, a strategy is sequential rational if it is an equilibrium at each stage.

Definition 11 A tupel $(\sigma, \Psi)$, consisting of a strategy profile and a belief system, is sequential rational if

- $\sigma$ is an ex-ante equilibrium with rectangular beliefs $\Psi_{i}^{0}$ and

- for all $t>0$ the tupel $\left(\sigma, \Psi^{t}\right)$ is an interim equilibrium with rectangular beliefs at stage $t$.

Now we can define Sequential Equilibrium.

Definition 12 The tupel $\left(\sigma^{*}, \Psi\right)$ consisting of a strategy profile and a belief system with $\Psi_{i}^{0}=\operatorname{rect}\left(\Phi_{\sigma_{-i}^{*}}^{0}\right)$ is a Sequential Equilibrium with rectangular beliefs if

- $\left(\sigma^{*}, \Psi\right)$ is sequential rational and

- $\Psi$ is consistent w.r.t. $\sigma$.

Theorem 4 There exists a Sequential Equilibrium with rectangular beliefs. 
Proof. Let $\epsilon^{k}, \Gamma^{k}$ and $\sigma^{k}$ as above. For each $\Gamma^{k}$ we can construct a belief system $\Psi^{k}$ that is stable under pasting, i.e. $\Psi_{i}^{t, k}\left(F_{i}^{t}\right)=\operatorname{rect}\left(\Phi_{\sigma_{-i}^{k}}^{t}\left(F_{i}^{t}\right)\right)$. Furthermore, by Theorem 1 there exists an ex-ante equilibrium $\hat{\sigma}^{k}$ with rectangular beliefs $\Psi^{0, k}$ for each $\Gamma^{k}$. Theorem 3 shows that $\left(\hat{\sigma}^{k}, \Psi^{t, k}\right)$ is an interim equilibrium with rectangular beliefs at stage t. By the compactness of the set of strategy profiles there exists a sub-sequence of $\sigma^{k}$ which converges to $\hat{\sigma}$. For this sub-sequence we can construct a system of rectangular beliefs $\Psi$ such that, $\Psi_{i}^{t}\left(F_{i}^{t}\right)=\lim _{k} \Psi_{i}^{t, k}\left(F_{i}^{t}\right)$. Then by construction $\Psi$ is stable under pasting and satisfies consistency w.r.t to $\hat{\sigma}$.

By Lemma $1 U_{i}^{e}(\sigma)$ and $U_{i}^{i}\left(\sigma, \Phi^{t}\right)$ are jointly continuous in $\sigma$. Then, since $\Sigma$ is the closure of $\cup_{k} \Sigma^{k}$ the strategy profile $\hat{\sigma}$ satisfies sequential rationality.

It is already known that ambiguity can generate new equilibria. The following example shows that under stability under pasting ambiguity can generate new Sequential Equilibria.

Example 1 This examples follows the idea of Greenberg (2000). There are three countries, country $A, B$ and $C$. Country $A$ and $B$ have to decide whether or not to reach a peace agreement. Country $C$ prefers a peace agreement and punishes country $A$ or $B$ if the peace negotiation fails. It cannot observe which of the two countries breakup the negotiation and has to decide which of the both countries would be punished without knowing who did not agree on the peace agreement. Country $A$ and $B$ choose to accept the peace agreement or not, without knowing which of them will be punished. In our setting nature takes the role of country $C$ and chooses the state of the world, "punish $A$ " or "punish B". The game is depicted in Figure 2.

First we show that without ambiguity there is no ex-ante equilibrium and therefore no Sequential Equilibrium in which the peace agreement take place. Let $\mu, \tilde{\mu}_{A}$ and $\tilde{\mu}_{B}$ represent the prior beliefs and the updated beliefs of country $A$ and $B$ respectively, in a game without ambiguity. Due to Bayes' rule and since neither country $A$ nor country $B$ learns the state it follows that $\mu=\tilde{\mu}_{A}=\tilde{\mu}_{B}$. Let $\alpha$ and $\beta$ denote the probability with which country $A$ and $B$ chooses war, respectively. Then the best responses are

$$
\beta^{*}= \begin{cases}1 & \text { if } 9 \mu>4 \\ {[0,1]} & \text { if } 9 \mu=4 \\ 0 & \text { if } 9 \mu<4\end{cases}
$$

and

$$
\alpha^{*}= \begin{cases}0 & \text { if } 5-2 \beta+3 \mu(\beta-3)<0 \\ {[0,1]} & \text { if } 5-2 \beta+3 \mu(\beta-3)=0 \\ 1 & \text { if } 5-2 \beta+3 \mu(\beta-3)>0\end{cases}
$$

In Section B.2 in the Appendix we calculate all ex-ante equilibria of this game without ambiguity and show that in all of them at least one of the countries $A$ or $B$ plays war with probability 1. To give country $A$ an incentive to play peace it has to belief that it will be 


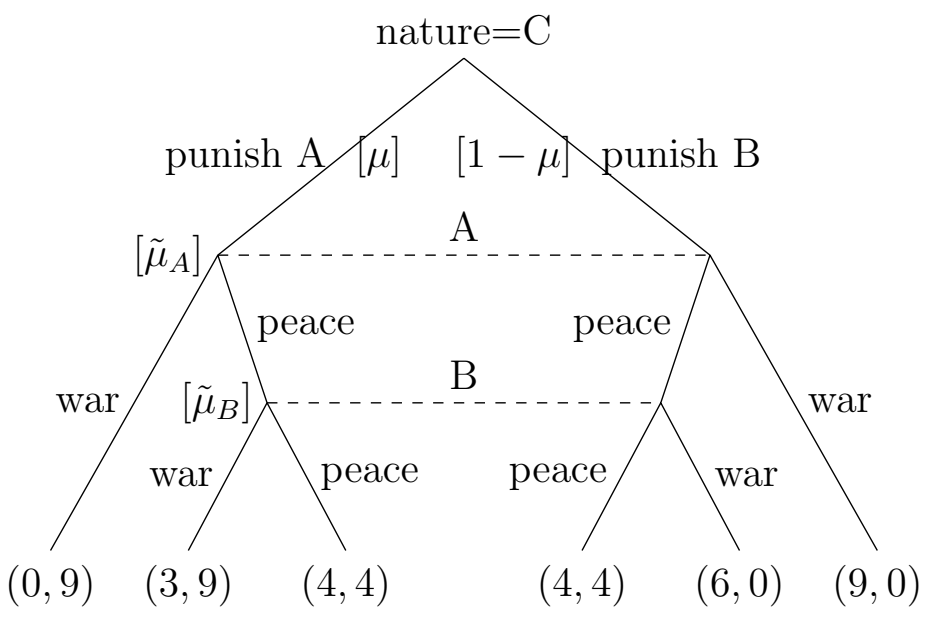

Figure 2: Example Peace Negotiation

punished with probability greater $\frac{5}{9}$, i.e. $\mu>\frac{5}{9}$. On the other hand to give country $B$ an incentive to play peace it has expect, that it will be punished with probability greater $\frac{5}{9}$, i.e. $\mu<\frac{4}{9}$. These two conditions contradict. Since there is no ex-ante equilibrium in which peace is played by both countries, there cannot exist a Sequential Equilibrium in which both countries choose peace.

With multiple priors, ambiguity induces a new equilibrium in which the peace agreement does not fail. Instead of one prior belief $\mu$ the players are faced with the imprecise probabilistic information $\mathcal{P}=[\underline{\mu}, \bar{\mu}]$. In Section B.2 we construct the prior belief sets of country $A$ and $B$ for $H=\{A w, A p w, A p p, B p p, B p w, B w\}$ where Apw denote the history where $A$ will be punished, country $A$ plays peace and country $B$ war. The belief sets are then given by

$\Phi_{\sigma_{-A}}^{0}=\{(\mu, \mu \beta, \mu(1-\beta),(1-\mu)(1-\beta),(1-\mu) \beta,(1-\mu))$ s.t. $\mu \in[\underline{\mu}, \bar{\mu}]\}$
$\Phi_{\sigma_{-B}}^{0}=\{(\mu \alpha, \mu(1-\alpha), \mu(1-\alpha),(1-\mu)(1-\alpha),(1-\mu)(1-\alpha),(1-\mu) \alpha)$ s.t. $\mu \in[\underline{\mu}, \bar{\mu}]\}$.

In the Appendix we show that $\Phi_{\sigma_{-A}}^{0}$ and $\Phi_{\sigma_{-B}}^{0}$ are stable under pasting. With ambiguity such that $\underline{\mu}<\frac{4}{9}$ and $\bar{\mu}>\frac{5}{9}$ there exists a sequential equilibrium in which both countries choose peace with probability 1 . Now, let $\epsilon_{i}^{k}$ be a sequence for each player that converges to 0 for $k \rightarrow \infty$ such that $\epsilon_{i}^{k}<\frac{1}{2}$ for $i=A, B$ and all $k$. Furthermore, let $\sum_{i}^{k}$ the set of strategies of country $i$ such that

$$
\begin{aligned}
& \Sigma_{A}^{k}=\left\{(\alpha, 1-\alpha) \text { s.t. } \alpha \in\left[\epsilon_{A}^{k}, 1-\epsilon_{A}^{k}\right]\right\}, \\
& \Sigma_{B}^{k}=\left\{(\beta, 1-\beta) \text { s.t. } \beta \in\left[\epsilon_{B}^{k}, 1-\epsilon_{B}^{k}\right]\right\} .
\end{aligned}
$$

In Section B.2 in the Appendix we show that given the restricted game with the set of 
strategies $\Sigma_{i}^{k}$ the optimal strategy of country $A$ and $B$ is to play $\alpha^{k}=\epsilon_{A}^{k}$ and $\beta^{k}=\epsilon_{B}^{k}$. The worst case belief of country $A$ is $\bar{\mu}>\frac{5}{9}$ for all strategies of country $A$ and B. Similarly $\mu<\frac{4}{9}$ is the worst case belief of country $B$ for all strategies of country $A$ and $B$. Therefore, both countries play war with the lowest possible probability. Since the strategy set is restricted to $\sum_{i}^{k}$ this probability is given by $\epsilon_{i}^{k}$. These considerations hold for any arbitrary $k$. For $k \rightarrow \infty$ the strategies of country $A$ and $B$ converge to the strategy playing peace with probability 1. Furthermore, Bayes' rule is always well defined for both players if $\alpha<1$, which is the case for $\alpha=\epsilon_{A}^{k}$ or $\alpha=0$. This implies consistency w.r.t $(\alpha=0, \beta=0)$ and proves the existence of a sequential equilibrium in which both countries play peace with probability 1.

\section{Conclusion and Discussion}

In this paper we introduce rectangularity to finite multi-stage extensive form games with ambiguous incomplete information. Players are faced with imprecise probabilistic information about states or types of the opponents. Furthermore, they know the information structure of the game. Given the imprecise probabilistic information and the knowledge about the information structure each player constructs a prior belief set which is rectangular. We show that rectangularity ensures dynamically consistent behavior in dynamic games and therefore existence of Sequential Equilibria. Furthermore, we show that in dynamic games with rectangular beliefs, ambiguity can create new Sequential Equilibria that do not exist in games without ambiguity.

\subsection{Discussion}

Singleton Subjective Beliefs: One could argue that our results could be obtained by choosing a single subjective prior belief for each player that equals the worst case ex-ante belief. Due to stability under pasting the worst case beliefs at the interim stages are the Bayesian updates of the worst case prior belief. Therefore, updating this single subjective prior belief would lead to the same beliefs at the interim stages as in the setting with a subjective set of beliefs. But the setting with single subjective beliefs would lack in an explanation how players derive their subjective beliefs. In our setting the subjective set of prior beliefs is endogenously derived from the common imprecise probabilistic information and the knowledge about the information structure of the game by assuming that players behave dynamically consistently, i.e. following stability under pasting. The choice of the worst case beliefs arise due to ambiguity aversion and MEU.

Hanany and Klibanoff (2007): Another way to rule out dynamic inconsistency in decision theoretic settings with multiple prior are the updating rules proposed by Hanany and Klibanoff (2007). They propose updating rules that update only a subset of the prior belief set using Bayes' rule. Which subset is updated depends on the optimal ex ante choice. Then, updating this subset of beliefs leads to an interim belief set that supports 
the ex ante optimal choice. This ensures dynamic consistency. However, comparing this approach to our model shows that the updating rules of Hanany and Klibanoff (2007) give a higher weight to the ex ante optimal choice. In our setting, players forecast their interim worst case belief and take them into account when construction their ex ante beliefs. Hence compared to the approach of Hanany and Klibanoff (2007), they are giving more weight to the interim optimal choice. We show in Section B.1.2 that the different approaches lead to a different dynamically consistent equilibrium in our running example.

An important assumption to use rectangularity is, that all players know the information structure. To use the updating rules of Hanany and Klibanoff (2007) agents only have to know the information that they are getting. But in games it is often assumed that the game tree and therefore the information structure is known by the players. If the players know which information they could get in the future it seems intuitive that they take this information into account when construction their ex ante belief sets. Hence, when defining models in a dynamically consistent MEU setting one has to decide which approach is better suited depending on the assumed information structure.

Ellsberg Games: As mentioned in the introduction Ellsberg games introduce ambiguous strategies, i.e. instead of playing a probability distribution over the pure strategies players can choose a set of probability distributions. In extensive form games with Ellsberg strategies, ambiguity is introduced due to the strategy of the players. In our setting ambiguity arises due to ambiguous information about types or states. This facilitate the definition of Sequential Equilibria since strategies are not ambiguous. In the conclusion of Muraviev, Riedel, and Sass (2017) an example illustrates the implications of their results for equilibrium concept in extensive form games with Ellsberg strategies. They show the existence of a dynamically consistent Ellsberg equilibrium for this example. However, a general formulation of equilibrium concepts for extensive form games with Ellsberg strategies and existency results are left for future research. Our results support their conjecture that rectangularity implies existence of dynamically consistent Ellsberg equilibria.

Games with two types: All examples that show the issue of dynamic inconsistency under MEU in games have at least three possible states or types. The reason for this is that dynamically inconsistent behavior cannot occur in games with only two types or states, i.e. $\left|H^{0}\right|=2$. With two types there are only two cases that can occur at an interim stage. Either the player learns the true type or not. In signaling games the first case correspond to a separating equilibria and the second to pooling equilibria. In Appendix $\mathrm{C}$ we explore both cases formally. If the player learns the true type updated beliefs are either 0 or 1. It is easy to show that then rectangularity is always satisfied. If the player does not learn the type Bayes' rule is always well defined. Furthermore, since there are only two types, the Bayesian update is monotone in the prior probability. This monotonicity implies dynamically consistent behavior for the second case. But as Example 1 shows, ambiguity also induces new Sequential Equilibria in dynamic games with 2 states or types. Hence, new equilibria may arise even if there is no dynamically inconsistent behavior. Therefore, there are two effects in games with dynamically inconsistent behavior. First ambiguity 
may change the set of equilibria, second dynamically inconsistent behavior may change the set of equilibria as well.

\section{References}

Gaurab Aryal and Ronald Stauber. A note on Kuhn's theorem with ambiguity averse players. Economics Letters, 125(1):110-114, 2014.

Subir Bose and Arup Daripa. A dynamic mechanism and surplus extraction under ambiguity. Journal of Economic theory, 144(5):2084-2114, 2009.

Subir Bose and Ludovic Renou. Mechanism design with ambiguous communication devices. Econometrica, 82(5):1853-1872, 2014.

Subir Bose, Emre Ozdenoren, and Andreas Pape. Optimal auctions with ambiguity. Theoretical Economics, 1(4):411-438, 2006.

Andrew Ellis. On dynamic consistency in ambiguous games. Games and Economic Behavior, 2018.

Daniel Ellsberg. Risk, ambiguity, and the savage axioms. The quarterly journal of economics, pages 643-669, 1961.

Larry G. Epstein and Martin Schneider. Recursive multiple-priors. Journal of Economic Theory, 113(1):1-31, 2003.

Johanna Etner, Meglena Jeleva, and Jean-Marc Tallon. Decision theory under ambiguity. Journal of Economic Surveys, 26(2):234-270, 2012.

Itzhak Gilboa. Theory of decision under uncertainty, volume 1. Cambridge university press Cambridge, 2009.

Itzhak Gilboa and Massimo Marinacci. Ambiguity and the bayesian paradigm. In Readings in Formal Epistemology, pages 385-439. Springer, 2016.

Itzhak Gilboa and David Schmeidler. Maxmin expected utility with non-unique prior. Journal of mathematical economics, 18(2):141-153, 1989.

Joseph Greenberg. The right to remain silent. Theory and Decision, 48(2):193-204, 2000.

Eran Hanany and Peter Klibanoff. Updating preferences with multiple priors. Theoretical Economics, 2:261-298, 2007.

Eran Hanany and Peter Klibanoff. Updating ambiguity averse preferences. The BE Journal of Theoretical Economics, 9(1), 2009. 
Eran Hanany, Peter Klibanoff, and Sujoy Mukerji. Incomplete information games with ambiguity averse players. mimeo, 2018.

Nengjiu Ju and Jianjun Miao. Ambiguity, learning, and asset returns. Econometrica, 80 (2):559-591, 2012.

Atsushi Kajii and Takashi Ui. Incomplete information games with multiple priors. The Japanese Economic Review, 56(3):332-351, 2005.

David M. Kreps and Robert Wilson. Sequential equilibria. Econometrica, 50(4):863894, 1982.

Hailin Liu and Wei Xiong. Dynamic consistency in incomplete information games under ambiguity. International Journal of Approximate Reasoning, 76:63-79, 2016.

Kin Chung Lo. Extensive form games with uncertainty averse players. Games and Economic Behavior, 28(2):256-270, 1999.

Igor Muraviev, Frank Riedel, and Linda Sass. Kuhn's theorem for extensive form ellsberg games. Journal of Mathematical Economics, 28:26-41, 2017.

Frank Riedel and Linda Sass. Ellsberg games. Theory and Decision, 76(4):469-509, 2014.

Frank Riedel, Jean-Marc Tallon, and Vassili Vergopoulos. Dynamic attitude towards imprecise probabilistic information. mimeo, 2017.

Rakesh Sarin and Peter P Wakker. Dynamic choice and nonexpected utility. Journal of Risk and Uncertainty, 17(2):87-120, 1998.

Alfredo di Tillio, Nenad Kos, and Matthias Messner. The design of ambiguous mechanisms. The Review of Economic Studies, 84(1):237-276, 2016.

\section{A Proofs}

\section{A.1 Proofs: Existence of Ex-Ante Equilibria}

Proof of Lemma 1. First consider the case where $\sigma_{-i}$ is completely mixed and Bayes' rule always well defined. Then any $\phi \in \operatorname{rect}\left(\Phi_{\sigma_{-i}}^{0}\right)$ has the following form

$$
\phi(h)=\sum_{F_{i}^{1} \in \mathcal{F}_{i}^{1}} \phi^{\prime}\left(F_{i}^{1}\right) \tilde{\phi}_{F_{i}^{1}}(h) .
$$


By Remark 2 we can assume without loss of generality, that $\phi^{\prime} \in \Phi_{\sigma_{-i}}^{0}$ and $\tilde{\phi}_{F_{i}^{1}} \in \Phi_{\sigma_{-i}}^{1}\left(F_{i}^{1}\right)$. Furthermore, Bayes' rule is always well defined. Therefore, there exist $\pi \in \mathcal{P}$ and $\pi_{F_{i}^{1}}^{\prime} \in \mathcal{P}$ for each $F_{i}^{1}$ such that

$$
\phi(h)=\sum_{F_{i}^{1} \in \mathcal{F}_{i}^{1}}\left(\sum_{h \in F_{i}^{1}} p_{\sigma_{-i}}\left(h \mid h^{0}\right) \pi\left(h^{0}\right)\right) \frac{p_{\sigma_{-i}}\left(h \mid h^{0}\right) \pi_{F_{i}^{1}}^{\prime}\left(h^{0}\right)}{\sum_{h \in F_{i}^{1}} p_{\sigma_{-i}}\left(h \mid h^{0}\right) \pi_{F_{i}^{1}}^{\prime}\left(h^{0}\right)} .
$$

With this formulation of $\phi$ and since $p_{\sigma_{-i}}(\cdot \mid \cdot)$ is completely characterized by $\sigma_{-i}$ we can now write $U_{i}^{e}$ as follows

$$
\begin{aligned}
& U_{i}^{e}(\sigma)=\min _{\phi \in \operatorname{rect}\left(\Phi_{\sigma_{-i}}^{0}\right)} \sum_{h \in H} u_{i}(h) p_{\sigma_{i}}\left(h \mid h^{0}\right) \phi(h) \\
& =\min _{\substack{\left(\pi,\left(\pi^{\prime}\right) \\
\in F_{i}^{1}\right)}} \sum_{h \in H} u_{i}(h) p_{\sigma_{i}}\left(h \mid h^{0}\right)\left(\sum_{F_{i}^{1} \in \mathcal{F}_{i}^{1}}\left(\sum_{h \in F_{i}^{1}} p_{\sigma_{-i}}\left(h \mid h^{0}\right) \pi\left(h^{0}\right)\right) \frac{p_{\sigma_{-i}}\left(h \mid h^{0}\right) \pi_{F_{i}^{1}}^{\prime}\left(h^{0}\right)}{\sum_{h \in F_{i}^{1}} p_{\sigma_{-i}}\left(h \mid h^{0}\right) \pi_{F_{i}^{1}}^{\prime}\left(h^{0}\right)}\right) \\
& =: \hat{U}_{i}^{e}\left(\left(\sigma_{i}, \sigma_{-i}\right)\right)
\end{aligned}
$$

Now we show that $\hat{U}_{i}^{e}\left(\left(\sigma_{i}, \sigma_{-i}\right)\right)$ is jointly continuous in $\left(\sigma_{i}, \sigma_{-i}\right)$ and therefore $U_{i}^{e}\left(\left(\sigma_{i}, \sigma_{-i}\right)\right)$ as well. First note, that

$$
\sum_{h \in H} u_{i}(h) p_{\sigma_{i}}\left(h \mid h^{0}\right)\left(\sum_{F_{i}^{1} \in \mathcal{F}_{i}^{1}}\left(\sum_{h \in F_{i}^{1}} p_{\sigma_{-i}}\left(h \mid h^{0}\right) \pi\left(h^{0}\right)\right) \frac{p_{\sigma_{-i}}\left(h \mid h^{0}\right) \pi_{F_{i}^{1}}^{\prime}\left(h^{0}\right)}{\sum_{h \in F_{i}^{1}} p_{\sigma_{-i}}\left(h \mid h^{0}\right) \pi_{F_{i}^{1}}^{\prime}\left(h^{0}\right)}\right)
$$

is continuous in $\left(\sigma_{i}, \sigma_{-i}\right)$. This implies that

$$
\begin{aligned}
& \forall \epsilon>0 \exists \delta>0 \text { s.t. }|a-b|<\delta \Rightarrow \\
& \mid \sum_{h \in H} u_{i}(h) p_{a_{i}}\left(h \mid h^{0}\right)\left(\sum_{F_{i}^{1} \in \mathcal{F}_{i}^{1}}\left(\sum_{h \in F_{i}^{1}} p_{a_{-i}}\left(h \mid h^{0}\right) \pi\left(h^{0}\right)\right) \frac{p_{a_{-i}}\left(h \mid h^{0}\right) \pi_{F_{i}^{1}}^{\prime}\left(h^{0}\right)}{\sum_{h \in F_{i}^{1}} p_{a_{-i}}\left(h \mid h^{0}\right) \pi_{F_{i}^{1}}^{\prime}\left(h^{0}\right)}\right) \\
& \quad-\sum_{h \in H} u_{i}(h) p_{b_{i}}\left(h \mid h^{0}\right)\left(\sum_{F_{i}^{1} \in \mathcal{F}_{i}^{1}}\left(\sum_{h \in F_{i}^{1}} p_{b_{-i}}\left(h \mid h^{0}\right) \pi\left(h^{0}\right)\right) \frac{p_{b_{-i}}\left(h \mid h^{0}\right) \pi_{F_{i}^{1}}^{\prime}\left(h^{0}\right)}{\sum_{h \in F_{i}^{1}} p_{b_{-i}}\left(h \mid h^{0}\right) \pi_{F_{i}^{1}}^{\prime}\left(h^{0}\right)}\right) \mid<\epsilon .
\end{aligned}
$$

By the compactness of $\mathcal{P}$ there exist $\left(\pi^{a},\left(\pi_{F_{i}^{1}}^{\prime, a}\right)_{F_{i}^{1}}\right)$ and $\left(\pi^{b},\left(\pi_{F_{i}^{1}}^{\prime, b}\right)_{F_{i}^{1}}\right)$ in $\mathcal{P} \times \mathcal{P}^{\left|\mathcal{F}_{i}^{1}\right|}$ such that

$$
\hat{U}_{i}^{e}(a)=\sum_{h \in H} u_{i}(h) p_{a_{i}}\left(h \mid h^{0}\right)\left(\sum_{F_{i}^{1} \in \mathcal{F}_{i}^{1}}\left(\sum_{h \in F_{i}^{1}} p_{a_{-i}}\left(h \mid h^{0}\right) \pi^{a}\left(h^{0}\right)\right) \frac{p_{a_{-i}}\left(h \mid h^{0}\right) \pi_{F_{i}^{1}}^{\prime, a}\left(h^{0}\right)}{\sum_{h \in F_{i}^{1}} p_{a_{-i}}\left(h \mid h^{0}\right) \pi_{F_{i}^{1}}^{\prime, a}\left(h^{0}\right)}\right)
$$


and

$$
\hat{U}_{i}^{e}(b)=\sum_{h \in H} u_{i}(h) p_{b_{i}}\left(h \mid h^{0}\right)\left(\sum_{F_{i}^{1} \in \mathcal{F}_{i}^{1}}\left(\sum_{h \in F_{i}^{1}} p_{b_{-i}}\left(h \mid h^{0}\right) \pi^{b}\left(h^{0}\right)\right) \frac{p_{b_{-i}}\left(h \mid h^{0}\right) \pi_{F_{i}^{1}}^{\prime, b}\left(h^{0}\right)}{\sum_{h \in F_{i}^{1}} p_{b_{-i}}\left(h \mid h^{0}\right) \pi_{F_{i}^{1}}^{\prime, b}\left(h^{0}\right)}\right) .
$$

Without loss of generality assume that $\hat{U}_{i}^{e}(a) \geq \hat{U}_{i}^{e}(b)$. Then $\forall \epsilon>0 \exists \delta>0$ such that $|a-b|<\delta$ it follows

$$
\begin{aligned}
& \left|\hat{U}_{i}^{e}(a)-\hat{U}_{i}^{e}(b)\right| \\
& \leq\left|\sum_{h \in H} u_{i}(h) p_{a_{i}}\left(h \mid h^{0}\right)\left(\sum_{F_{i}^{1} \in \mathcal{F}_{i}^{1}}\left(\sum_{h \in F_{i}^{1}} p_{a_{-i}}\left(h \mid h^{0}\right) \pi^{b}\left(h^{0}\right)\right) \frac{p_{a_{-i}}\left(h \mid h^{0}\right) \pi_{F_{i}^{1}}^{\prime, b}\left(h^{0}\right)}{\sum_{h \in F_{i}^{1}} p_{a_{-i}}\left(h \mid h^{0}\right) \pi_{F_{i}^{1}}^{\prime, b}\left(h^{0}\right)}\right)-\hat{U}_{i}^{e}(b)\right| \\
& <\epsilon .
\end{aligned}
$$

The first inequality holds since $\left(\pi^{b}, \pi^{\prime, b}\right)$ is in general not a worst case belief given $a$. The second inequality follows by the continuity of Equation 3 in $\left(\sigma_{i}, \sigma_{-i}\right)$.

Hence, $\hat{U}_{i}^{e}(\sigma)$ is jointly continuous in $\sigma=\left(\sigma_{i}, \sigma_{-i}\right)$ for completely mixed $\sigma_{-i}$.

If $\sigma_{-i}$ is not completely mixed and Bayes' rule not well defined for some $F_{i}^{1}$, then the denominator $\sum_{h \in F_{i}^{1}} \phi_{F_{i}^{1}}^{\prime}(h)=0$. But this is equivalent to $\phi\left(F_{i}^{1}\right)=0 .{ }^{13}$ Therefore,

$$
\begin{aligned}
& \hat{U}_{i}^{e}\left(\left(\sigma_{i}, \sigma_{-i}\right)\right) \\
& =\min _{\substack{\left(\pi,\left(\pi^{\prime}\right)_{F_{i}^{1}}\right) \\
\in \mathcal{P} \times \mathcal{P}^{\left|\mathcal{F}_{i}^{1}\right|}}} \sum_{h \in H} u_{i}(h) p_{\sigma_{i}}\left(h \mid h^{0}\right)\left(\sum_{\substack{F_{i}^{1} \in \mathcal{F}_{i}^{1} \\
\text { s.t. } \phi\left(F_{i}^{1}\right) \neq 0}}\left(\sum_{h \in F_{i}^{1}} p_{\sigma_{-i}}\left(h \mid h^{0}\right) \pi\left(h^{0}\right)\right) \frac{p_{\sigma_{-i}}\left(h \mid h^{0}\right) \pi_{F_{i}^{1}}^{\prime}\left(h^{0}\right)}{\sum_{h \in F_{i}^{1}} p_{\sigma_{-i}}\left(h \mid h^{0}\right) \pi_{F_{i}^{1}}^{\prime}\left(h^{0}\right)}\right) .
\end{aligned}
$$

and we can ignore $F_{i}^{1}$ where Bayes' rule is not well defined.

Now we can prove Theorem 1.

Proof of Theorem 1. First remember that the set of histories $H$, the set of information sets $\mathcal{I}$, the set of actions for each player at each information set $A_{i}\left(I_{i}^{t}\right)$ and the set of players $N$ are finite. A behaviour strategy of player $i$ was defined such that $\sigma_{i}\left(I_{i}^{t}\right) \in \Delta\left(A_{I}\left(I_{i}^{t}\right)\right)$. The set of strategies of agent $i$ is then $\Sigma_{i}=X_{I_{i}^{t} \in \mathcal{I}_{i}} \Delta\left(A_{i}\left(I_{i}^{t}\right)\right)$ and the set of strategy profiles $\Sigma=\mathrm{X}_{i \in N} \Sigma_{i}$. We define the best response of player $i$ given the strategy of the opponents $\sigma_{-i}$ as the correspondence $B_{i}: \Sigma_{-i} \rightarrow \Sigma_{i}$ with

$$
B_{i}\left(\sigma_{-i}\right)=\left\{\sigma_{i} \in \Sigma_{i}: \sigma_{i} \in \operatorname{argmax}_{\sigma_{i} \in \Sigma_{i}} U_{i}^{e}\left(\sigma_{i}, \sigma_{-i}\right)\right\} .
$$

\footnotetext{
${ }^{13}$ This equivalence follows from the full support assumption of $\mathcal{P}$. Since $\pi\left(h^{0}\right)>0$ for all $h^{0}$ and all $\pi \in \mathcal{P}$ an information set has only probability 0 if the transition probability of all histories contained in this information set are 0 . This implies that $p_{\sigma_{-i}}\left(h \mid h^{0}\right) \pi\left(h^{0}\right)=0$ for all $\pi \in \mathcal{P}$.
} 
Then the correspondence $B: \Sigma \rightarrow \Sigma$ with

$$
B(\sigma)=\underset{i \in N}{\chi} B_{i}\left(\sigma_{-i}\right)
$$

defines the best response.

We will use Kakutani's fixed point theorem to show that $B$ has a fixed point and therefore the existence of an ex-ante equilibrium. To apply Kakutani's fixed point theorem we need the following conditions:

i) $\Sigma$ is non-empty, convex and compact

ii) $B: \Sigma \rightarrow \Sigma$ is a upper-hemicontinuous correspondence and $B(\sigma)$ is non-empty and closed $\forall \sigma \in \Sigma$

iii) $B(\sigma)$ is convex $\forall \sigma \in \Sigma$

We will show this conditions step by step:

i) Since $\mathcal{I}_{i}$ and $A_{i}\left(I_{i}^{t}\right)$ are finite for all information sets $I_{i}^{t}$ and all player $i, \Delta\left(A_{i}\left(I_{i}^{t}\right)\right)$ is non-empty, compact and convex. Therefore, $\Sigma_{i}$ and $\Sigma$ are non-empty, compact and convex as well.

ii) To show the second point we use Berge's maximum theorem.

Let $C: \Sigma_{-i} \rightarrow \Sigma_{i}$ be a correspondence such that $C\left(\sigma_{-i}\right)=\Sigma_{i}$ for all $\sigma_{-i}$. Then $C$ is upper and lower hemicontinuous as the following explanation shows and therefore continuous.

The definition of lower hemicontinuity says: $C$ is lower hemicontinuous at $a$ if for all open sets $V$ intersecting $C(a)$ exists a neighbourhood $U$ of $a$ such that $C(x)$ intersects $V$ for all $x \in U$. Since $C(a)=\Sigma_{i}=C(x)$ for all $x \in \Sigma_{-i}$ the definition is satisfied for each $a, U$ and $V$.

For upper hemicontinuity we use the graph theoretic characterization: Let $\operatorname{Gr}(C):=$ $\left\{(a, b) \in \Sigma_{-i} \times \Sigma_{i}\right.$ such that $\left.b \in C(a)\right\}$. If $\Sigma_{i}$ is compact and $\operatorname{Gr}(C)$ closed, $C: \Sigma_{-i} \rightarrow$ $\Sigma_{i}$ is a upper hemicontinuous correspondence with closed domain and closed values. By the definition of $C$ it follows that $\operatorname{Gr}(C)=\Sigma_{-i} \times \Sigma_{i} . \Sigma_{i}$ and $\Sigma_{-i}$ are compact by i) and therefore closed. Hence, $C$ is upper hemicontinuous.

Now we can apply Berge's maximum theorem: With our notation

$$
\begin{gathered}
U_{i}^{e}: \Sigma_{i} \times \Sigma_{-i} \rightarrow \mathbb{R}, \\
C: \Sigma_{-i} \rightarrow \Sigma_{i} \quad \text { s.t } C\left(\sigma_{-i}\right)=\Sigma, \\
C^{*}\left(\sigma_{-i}\right):=\operatorname{argmax}\left\{U_{i}^{e}\left(\sigma_{i}, \sigma_{-i}\right) \text { such that } \sigma_{i} \in C\left(\sigma_{-i}\right)=\Sigma_{i}\right\}=B_{i}\left(\sigma_{-i}\right) .
\end{gathered}
$$

Berge's maximum theorem states that if $U_{i}^{e}$ is jointly continuous in both arguments and $C$ is continuous in $\sigma_{-i}$ then $C^{*}$ is non-empty, convex valued and upper hemicontinuous in $\sigma_{-i}$. Hence, by Lemma $1 B_{i}$ is a upper-hemicontinuous correspondence and $B_{i}\left(\sigma_{-i}\right)$ 
is non-empty and closed $\forall \sigma_{-i} \in \Sigma_{-i}$. Since $B(\sigma)=\chi_{i \in N} B_{i}\left(\sigma_{-i}\right)$ the same holds for $B$.

iii) To show the convexity of $B(\sigma)$ we first show that $U_{i}^{e}$ is concave in $\sigma_{i}$. Let $\bar{\sigma}_{i}$ and $\tilde{\sigma}_{i} \in \Sigma_{i}$ and $\alpha \in[0,1]$.

$$
\begin{aligned}
& U_{i}^{e}\left(\alpha \bar{\sigma}_{i}+(1-\alpha) \tilde{\sigma}, \sigma_{-i}\right)=\min _{\phi \in \operatorname{rect}\left(\Phi_{\sigma_{-i}}^{0}\right)} \sum_{h \in H} u_{i}(h) p_{\alpha \bar{\sigma}_{i}+(1-\alpha) \tilde{\sigma}}\left(h \mid h^{0}\right) \phi(h) \\
& \quad=\min _{\phi \in \operatorname{rect}\left(\Phi_{\sigma_{-i}}^{0}\right)} \sum_{h \in H} u_{i}(h) \prod_{t=0}^{T}(\alpha \underbrace{\bar{\sigma}_{i}\left(I_{i}\left(h^{t}\right)\right)\left(h_{t, i}\right)}_{\geq 0}+(1-\alpha) \underbrace{\tilde{\sigma}_{i}\left(I_{i}\left(h^{t}\right)\right)\left(h_{t, i}\right)}_{\geq 0}) \phi(h) \\
& \quad \geq \min _{\phi \in \operatorname{rect}\left(\Phi_{\sigma_{-i}}^{0}\right)} \sum_{h \in H} u_{i}(h)\left(\prod_{t=0}^{T} \alpha \bar{\sigma}_{i}\left(I_{i}\left(h^{t}\right)\right)\left(h_{t, i}\right)+\prod_{t=0}^{T}(1-\alpha) \tilde{\sigma}_{i}\left(I_{i}\left(h^{t}\right)\right)\left(h_{t, i}\right)\right) \phi(h) \\
& \quad=\min _{\phi \in \operatorname{rect}\left(\Phi_{\sigma_{-i}}^{0}\right)}\left(\alpha \sum_{h \in H} u_{i}(h) p_{\bar{\sigma}_{i}}\left(h \mid h^{0}\right) \phi(h)+(1-\alpha) \sum_{h \in H} u_{i}(h) p_{\tilde{\sigma}_{i}}\left(h \mid h^{0}\right) \phi(h)\right) \\
& \quad \geq \alpha \min _{\phi \in \operatorname{rect}\left(\Phi_{\sigma_{-i}}^{0}\right)} \sum_{h \in H} u_{i}(h) p_{\bar{\sigma}_{i}}\left(h \mid h^{0}\right) \phi(h)+(1-\alpha) \min _{\phi \in \operatorname{rect}\left(\Phi_{\sigma_{-i}}^{0}\right)} \sum_{h \in H} u_{i}(h) p_{\tilde{\sigma}_{i}}\left(h \mid h^{0}\right) \phi(h) \\
& \quad=\alpha U_{i}^{e}\left(\bar{\sigma}_{i}, \sigma_{-i}\right)+(1-\alpha) U_{i}^{e}\left(\tilde{\sigma}_{i}, \sigma_{-i}\right) .
\end{aligned}
$$

With the concavity of $U_{i}^{e}$ we can prove that $B_{i}\left(\sigma_{-i}\right)$ is convex for all $\sigma_{-i}$. Fix some arbitrary $\sigma_{-i}$ and let $\bar{\sigma}_{i}, \tilde{\sigma}_{i} \in B_{i}\left(\sigma_{-i}\right)$ and $\alpha \in[0,1]$. We have to show, that $\alpha \bar{\sigma}_{i}+(1-$ $\alpha) \tilde{\sigma}_{i} \in B_{i}\left(\sigma_{-i}\right)=\operatorname{argmax}_{\sigma_{i} \in \Sigma_{i}} U_{i}^{e}\left(\sigma_{i}, \sigma_{-i}\right)$. Since $\bar{\sigma}_{i}, \tilde{\sigma}_{i} \in B_{i}\left(\sigma_{-i}\right)$ it follows that

$$
\begin{aligned}
U_{i}^{e}\left(\bar{\sigma}_{i}, \sigma_{-i}\right)=U_{i}^{e}\left(\tilde{\sigma}_{i}, \sigma_{-i}\right) & =\max _{\sigma_{i} \in \Sigma_{i}} U_{i}^{e}\left(\sigma_{i}, \sigma_{-i}\right) \\
& \geq U_{i}^{e}\left(\alpha \bar{\sigma}_{i}+(1-\alpha) \tilde{\sigma}_{i}, \sigma_{-i}\right) \\
& \geq \alpha U_{i}^{e}\left(\bar{\sigma}_{i}, \sigma_{-i}\right)+(1-\alpha) U_{i}^{e}\left(\tilde{\sigma}_{i}, \sigma_{-i}\right) \\
& =\max _{\sigma_{i} \in \Sigma_{i}} U_{i}^{e}\left(\sigma_{i}, \sigma_{-i}\right)
\end{aligned}
$$

where the last inequality follows from the concavity of $U_{i}^{e}$. Then

$$
\begin{aligned}
U_{i}^{e}\left(\alpha \bar{\sigma}_{i}+(1-\alpha) \tilde{\sigma}_{i}, \sigma_{-i}\right) & =\max _{\sigma_{i} \in \Sigma_{i}} U_{i}^{e}\left(\sigma_{i}, \sigma_{-i}\right) \\
\Rightarrow \quad \alpha \bar{\sigma}_{i}+(1-\alpha) \tilde{\sigma}_{i} & \in \operatorname{argmax}_{\sigma_{i} \in \Sigma_{i}} U_{i}^{e}\left(\sigma_{i}, \sigma_{-i}\right)=B_{i}\left(\sigma_{-i}\right) .
\end{aligned}
$$

Hence, $B_{i}\left(\sigma_{-i}\right)$ is convex valued for all $\sigma_{-i} \in \Sigma_{-i}$. Since this is true for all $i \in N$ it follows that $B(\sigma)$ is convex valued for all $\sigma \in \Sigma$.

Now we can apply Kakutanis fixed point theorem which shows that the best response correspondence $B$ has a fixed point and therefore proves the existence of an ex-ante equilibrium with rectangular beliefs. 


\section{A.2 Proofs: Relation of Ex-Ante and Interim Equilibria}

Proof of Lemma 2. The proof consist of two steps:

i) First we show that

$$
\begin{aligned}
& \sum_{h \in F_{i}^{t-1}} u_{i}(h) p_{\sigma_{i}}\left(h \mid h^{t-1}\right) \phi^{*}(h) \\
& \quad \geq \sum_{F_{i}^{t} \in \mathcal{F}_{i}^{t}} \phi^{*}\left(F_{i}^{t}\right) p_{\sigma_{i}}\left(h^{t} \mid h^{t-1}\right) \min _{\tilde{\phi} \in \operatorname{rect}\left(\Phi_{\sigma_{-i}}^{t}\left(F_{i}^{t}\right)\right)} \sum_{h \in H} u_{i}(h) p_{\sigma_{i}}\left(h \mid h^{t}\right) \tilde{\phi}(h)
\end{aligned}
$$

which follows directly from Bayesian updating. Take into account, that $\phi^{*}\left(F_{i}^{t}\right)=0$ is equivalent to $\phi^{*}(h)=0 \quad \forall h \in F_{i}^{t}$.

$$
\begin{aligned}
\sum_{h \in F_{i}^{t-1}} u_{i}(h) p_{\sigma_{i}}\left(h \mid h^{t-1}\right) \phi^{*}(h)=\sum_{F_{i}^{t} \in \mathcal{F}_{i}^{t}} \sum_{h \in F_{i}^{t}} u_{i}(h) p_{\sigma_{i}}\left(h \mid h^{t-1}\right) \phi^{*}(h) \\
=\sum_{\substack{F_{i}^{t} \in \mathcal{F}_{i}^{t} \\
\text { s.t. } \phi^{*}\left(F_{i}^{t}\right)>0}} \frac{\phi^{*}\left(F_{i}^{t}\right)}{\phi^{*}\left(F_{i}^{t}\right)} \sum_{h \in F_{i}^{t}} u_{i}(h) p_{\sigma_{i}}\left(h \mid h^{t-1}\right) \phi^{*}(h) \\
=\sum_{F_{i}^{t} \in \mathcal{F}_{i}^{t}} \phi^{*}\left(F_{i}^{t}\right) \sum_{h \in F_{i}^{t}} u_{i}(h) p_{\sigma_{i}}\left(h \mid h^{t-1}\right) \underbrace{\frac{\phi^{*}(h)}{\phi^{*}\left(F_{i}^{t}\right)}}_{\underbrace{}_{F_{i}^{t} \in \mathcal{F}_{i}^{t}}} \\
=\sum_{F_{i}^{t} \in \mathcal{F}_{i}^{t}} \phi^{*}\left(F_{i}^{t}\right) p_{\sigma_{i}}\left(h^{t} \mid h^{t-1}\right) \\
\geq \sum_{\tilde{\phi} \in \operatorname{rect}\left(\Phi_{\sigma_{-i}}^{t}\left(F_{i}^{t}\right)\right)} \min _{\left.\min _{-i}\left(F_{i}^{t}\right)\right)} \sum_{h \in F_{i}^{t}} u_{i}(h) p_{\sigma_{i}}\left(h \mid h^{t}\right) \tilde{\phi}(h)
\end{aligned}
$$

where the last equality follows since $p_{\sigma_{i}}\left(h^{t} \mid h^{t-1}\right)=p_{\sigma_{i}}\left(\bar{h}^{t} \mid \bar{h}^{t-1}\right)$ for all $h, \bar{h}$ in $F_{i}^{t}$.

ii) For the other direction

$$
\begin{aligned}
& \sum_{h \in F_{i}^{t-1}} u_{i}(h) p_{\sigma_{i}}\left(h \mid h^{t-1}\right) \phi^{*}(h) \\
& \quad \leq \sum_{F_{i}^{t} \in \mathcal{F}_{i}^{t}} \phi^{*}\left(F_{i}^{t}\right) p_{\sigma_{i}}\left(h^{t} \mid h^{t-1}\right) \min _{\tilde{\phi} \in \operatorname{rect}\left(\Phi_{\sigma_{-i}}^{t}\left(F_{i}^{t}\right)\right)} \sum_{h \in F_{i}^{t}} u_{i}(h) p_{\sigma_{i}}\left(h \mid h^{t}\right) \tilde{\phi}(h)
\end{aligned}
$$

let $\tilde{\phi}_{F_{i}^{t}}^{*} \in \operatorname{argmin}_{\tilde{\phi} \in \operatorname{rect}\left(\Phi_{\sigma_{-i}}^{t}\left(F_{i}^{t}\right)\right)} \sum_{h \in H} u_{i}(h) p_{\sigma_{i}}\left(h \mid h^{t}\right) \tilde{\phi}(h)$ for all $F_{i}^{t}$ and $\phi^{*}$ as above. Stability under pasting implies that there exist a $\phi^{\prime} \in \Phi_{\sigma_{-i}}^{t-1}\left(F_{i}^{t-1}\right)$ such that

$$
\phi^{\prime}(h)=\sum_{F_{i}^{t} \in \mathcal{F}_{i}^{t}} \phi^{*}\left(F_{i}^{t}\right) \tilde{\phi}_{F_{i}^{t}}^{*}(h)
$$


which in general is not a worst case belief. Then

$$
\begin{aligned}
\sum_{h \in F_{i}^{t-1}} u_{i}(h) p_{\sigma_{i}}\left(h \mid h^{t-1}\right) \phi^{*}(h) & \leq \sum_{h \in F_{i}^{t-1}} u_{i}(h) p_{\sigma_{i}}\left(h \mid h^{t-1}\right) \phi^{\prime}(h) \\
& =\sum_{h \in F_{i}^{t-1}} u_{i}(h) p_{\sigma_{i}}\left(h \mid h^{t-1}\right) \sum_{F_{i}^{t} \in \mathcal{F}_{i}^{t}} \phi^{*}\left(F_{i}^{t}\right) \tilde{\phi}^{*}(h) \\
& =\sum_{F_{i}^{t} \in \mathcal{F}_{i}^{t}} \phi^{*}\left(F_{i}^{t}\right) \sum_{h \in F_{i}^{t}} u_{i}(h) p_{\sigma_{i}}\left(h \mid h^{t-1}\right) \tilde{\phi}^{*}(h) \\
& =\sum_{F_{i}^{t} \in \mathcal{F}_{i}^{t}} \phi^{*}\left(F_{i}^{t}\right) p_{\sigma_{i}}\left(h^{t} \mid h^{t-1}\right) \min _{\tilde{\phi} \in \Phi_{\sigma_{-i}}^{t}\left(F_{i}^{t}\right)} \sum_{h \in F_{i}^{t}} u_{i}(h) p_{\sigma_{i}}\left(h \mid h^{t}\right) \tilde{\phi}(h) .
\end{aligned}
$$

Combining step i) and ii) proves Lemma 2

\section{Proof of Theorem 3 (ex-ante implies interim).}

We show that for an arbitrary $t$ a completely mixed interim equilibrium with rectangular beliefs at $t-1$ implies an interim equilibrium with rectangular beliefs at $t$. Then the theorem follows from iteration.

Let $\left(\sigma^{*}, \Psi^{t-1}\right)$ be a completely mixed interim equilibrium with rectangular beliefs at $t-1$ and assume that $\left(\sigma^{*}, \operatorname{rect}\left(\Phi_{\sigma_{-i}}^{t}\right)\right)$ is not an interim equilibrium at $t$. Hence, there exist a player $i$, an information set $F_{i}^{t}$ and a strategy profile $\left(\sigma_{i}^{\prime}, \sigma_{-i}^{*}\right)$ where player $i$ deviates from $\sigma^{*}$. Let $F_{i}^{t-1}$ be the information set that precedes $F_{i}^{t}$ and $\phi^{*}$ the worst case belief at $F_{i}^{t-1}$ given $\sigma_{-i}^{*}$, i.e.

$$
\phi^{*} \in \operatorname{argmin}_{\phi \in \operatorname{rect}\left(\Phi_{\sigma_{-i}^{*}}^{t-1}\left(F_{i}^{t-1}\right)\right)} \sum_{h \in F_{i}^{t-1}} u_{i}(h) p_{\sigma_{i}^{*}}\left(h \mid h^{t-1}\right) \phi(h) .
$$

Similarly, let $\phi^{*, t}$ denote the worst case belief at $F_{i}^{t}$ given $\sigma_{i}^{*}$ and $\bar{\phi}$ denote the worst case at $F_{i}^{t-1}$ belief given the strategy $\sigma_{i}$ which equals $\sigma_{i}^{\prime}$ for $s \geq t$ and equals $\sigma_{i}^{*}$ for $s<t$. Furthermore, let $\phi^{\prime}$ be the pasting of $\bar{\phi}$ and $\phi^{*, t}$. Then $\phi^{\prime}$ is in general not a worst case belief and similar to step ii) of the proof of Lemma 2 it follows

$$
\begin{aligned}
U_{i}^{i}\left(\sigma^{*}, \Psi^{t-1}\left(F_{i}^{t-1}\right)\right) & \leq \sum_{h \in H} u_{i}(h) p_{\sigma_{i}^{*}}\left(h \mid h^{t-1}\right) \phi^{\prime}(h) \\
& =\sum_{F_{i}^{t} \in \mathcal{F}_{i}^{t}} \bar{\phi}\left(F_{i}^{t}\right) p_{\sigma_{i}^{*}}\left(h^{t} \mid h^{t-1}\right) U_{i}^{i}\left(\sigma^{*}, \operatorname{rect}\left(\Phi_{\sigma_{-i}^{*}}^{t}\left(F_{i}^{t}\right)\right)\right)
\end{aligned}
$$

By our assumption $\left(\sigma^{*}, \operatorname{rect}\left(\Phi_{\sigma_{-i}^{*}}^{t}\right)\right)$ is not an interim equilibrium at $t$, i.e. there exist $F_{i}^{t}$ and $\sigma_{i}^{\prime}$ such that

$$
U_{i}^{i}\left(\sigma^{*}, \operatorname{rect}\left(\Phi_{\sigma_{-i}^{*}}^{t}\left(F_{i}^{t}\right)\right)\right)<U_{i}^{i}\left(\left(\sigma_{i}^{\prime}, \sigma_{-i}^{*}\right), \operatorname{rect}\left(\Phi_{\sigma_{-i}^{*}}^{t}\left(F_{i}^{t}\right)\right)\right)
$$


Furthermore, since $\sigma^{*}$ is completely mixed and $\mathcal{P}$ has full support it follows that

$$
\bar{\phi}\left(F_{i}^{t}\right) p_{\sigma_{i}^{*}}\left(h^{t} \mid h^{t-1}\right)>0 \quad \forall F_{i}^{t}
$$

Combining Equation 4, Equation 5 and Equation 6 leads to

$$
\begin{aligned}
U_{i}^{i}\left(\sigma^{*}, \Psi^{t-1}\left(F_{i}^{t-1}\right)\right) & <\sum_{F_{i}^{t} \in \mathcal{F}_{i}^{t}} \bar{\phi}\left(F_{i}^{t}\right) p_{\sigma_{i}^{*}}\left(h^{t} \mid h^{t-1}\right) U_{i}^{i}\left(\left(\sigma_{i}^{\prime}, \sigma_{-i}^{*}\right), \operatorname{rect}\left(\Phi_{\sigma_{-i}^{*}}^{t}\left(F_{i}^{t}\right)\right)\right) \\
& =\min _{\phi \in \operatorname{rect}\left(\Phi_{\sigma_{-i}^{t-1}}^{t}\right)} \sum_{h \in H} u_{i}(h) p_{\sigma_{i}^{*}}\left(h^{t} \mid h^{t-1}\right) p_{\sigma_{i}^{\prime}}\left(h \mid h^{t}\right) \phi(h)
\end{aligned}
$$

For the equality in Equation 7 consider, that by construction and Remark 4 the pasting of $\bar{\phi}$ and the interim worst case belief at $t$ given $\sigma_{i}^{\prime}$, is the worst case belief at $t-1$ given the strategy $\sigma_{i}$ which equals $\sigma_{i}^{\prime}$ for $s \geq t$ and equals $\sigma_{i}^{*}$ for $s<t$. Then Equation 7 follows from Lemma 2.

The calculation above form a contradiction since $\left(\sigma^{*}, \Psi^{t-1}\right)$ is an interim equilibrium with rectangular beliefs at stage $t-1$. Hence, $\left(\sigma^{*}, \operatorname{rect}\left(\Phi_{\sigma^{*}}^{t}\right)\right)$ is an interim equilibrium with rectangular beliefs at stage $t$.

\section{B Examples}

\section{B.1 Running Example}

\section{B.1.1 Dynamically Inconsistent Behavior without Stability under Pasting}

Example 2 Aryal and Stauber (2014) show that in the example, depicted in Figure 3, stability under pasting is a necessary condition for dynamically consistent behavior. We use this example as a running example during the paper. Here we will show that without

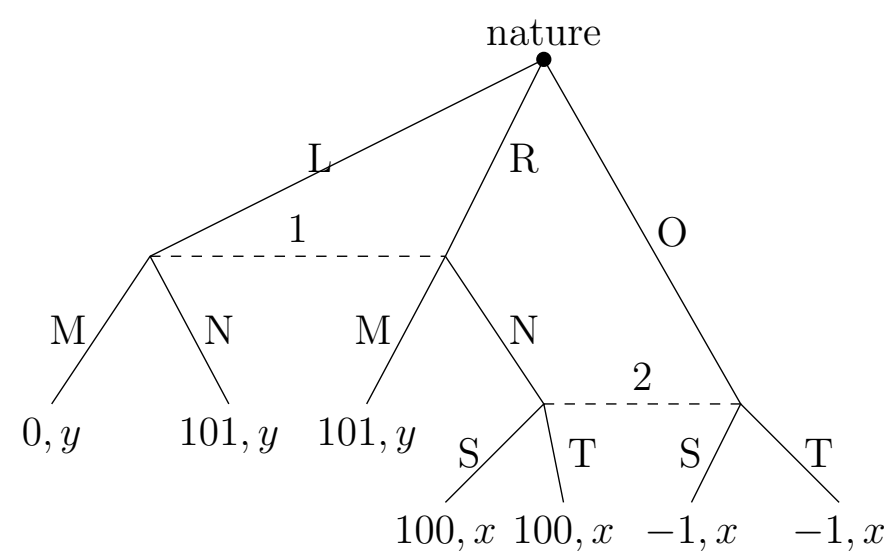

Figure 3: Aryal and Stauber 3 player game 
ambiguity player 1 behaves dynamically inconsistently.

The imprecise probabilistic information is given by

$$
\mathcal{P}=\{(1-\epsilon)(0,1,0)+\epsilon(l, r, o):(l, r, o) \in \Delta\}
$$

where $l, r$ and $o$ denote the probabilities of the types $L, R$ and $O$ respectively. Furthermore, let $(n, m)$ denote a strategy of player 1 where $n=1-m$ and $m$ denotes the probability of playing $M$. Similarly $(s, t)$ is the strategy of player 2 .

At the end of Section 2.2 we show how to construct the set of histories, the information partition and the prior belief sets. Remember that this leads to the following results.

$$
\begin{aligned}
H & =\{L M, L N, R M, R N S, R N T, O S, O T\}, \\
\mathcal{F}_{i}^{0} & =H, \\
\mathcal{F}_{1}^{1} & =\left\{F_{1,1}^{1}, F_{1,2}^{1}, F_{1,3}^{1}\right\}=\{\{L M, L N, R M, R N S, R N T\},\{O S\},\{O T\}\}, \\
\mathcal{F}_{2}^{1} & =\left\{F_{2,1}^{1}, F_{2,2}^{1}, F_{2,3}^{1}, F_{2,4}^{1}\right\}=\{\{R N S, R N T, O S, O T\},\{L M\},\{L N\},\{R M\}\}, \\
\Phi_{(s, t)}^{0} & =\{(l, l, r, r s, r t, o s, o t) \text { s.t. }(l, r, o) \in \mathcal{P}\}, \\
\Phi_{(m, n)}^{0} & =\{(l m, l n, r m, r n, r n, o, o) \text { s.t. }(l, r, o) \in \mathcal{P}\} .
\end{aligned}
$$

We now show that dynamic consistency is violated without stability under pasting

The ex-ante optimal choice of player 1 is:

$$
\begin{aligned}
& \max _{(1-m, m)} U^{e}((1-m, m))=\max _{(1-m, m)} \min _{\phi \in \Phi^{0}} 101(1-m) \phi(L N)+101 m \phi(R M)+100(1-m) \phi(R N S) \\
& \quad+100(1-m) \phi(R N T)-\phi(O S)-\phi(O T) \\
& =\max _{(1-m, m)} \min _{(l, r, o) \in \mathcal{P}} 101(1-m) l+101 m r+100(1-m) r(s+t)-(1-r-l)(s+t) \\
& =\max _{(1-m, m)} \min _{(l, r, o) \in \mathcal{P}} 101(1-m) l+101 m r+100(1-m) r-(1-r-l) \\
& =\max _{(1-m, m)} \min _{(l, r, o) \in \mathcal{P}} l \underbrace{(100-101 m)}_{>0}+r \underbrace{(101+m)}_{>0}-1 \\
& \Rightarrow \text { the ex-ante worst case belief is }(l, r, o)=(0,1-\epsilon, \epsilon) \\
& \Rightarrow \max _{(1-m, m)} U^{e}((1-m, m))=\max _{(1-m, m)}(1-\epsilon)(101+m)-1 \\
& \Rightarrow m^{*}=1 .
\end{aligned}
$$

Now we show that $m^{*}=1$ is not an interim equilibrium given Bay $\left(\Phi^{0}\right)$. The prior by prior Bayesian update of $\Phi^{0}$ is

$$
\begin{aligned}
\operatorname{Bay}\left(\Phi_{(s, t)}^{0} \mid F_{1,1}^{1}\right) & =\left\{\left(\frac{l}{2 l+2 r}, \frac{l}{2 l+2 r}, \frac{r}{2 l+2 r}, \frac{r s}{2 l+2 r}, \frac{r t}{2 l+2 r}, 0,0\right) \text { s.t. }(l, r, o) \in \mathcal{P}\right\} \\
& =\left\{(\tilde{l}, \tilde{l}, \tilde{r}, \tilde{r} s, \tilde{r} t, 0,0) \text { s.t. } \tilde{l} \in\left[0, \frac{\epsilon}{2}\right], \tilde{r} \in\left[\frac{1-\epsilon}{2}, \frac{1}{2}\right], 2 \tilde{l}+\tilde{r}+s \tilde{r}+t \tilde{r}=1\right\}
\end{aligned}
$$




$$
\begin{aligned}
& =\left\{(\tilde{l}, \tilde{l}, \tilde{r}, \tilde{r} s, \tilde{r} t, 0,0) \text { s.t. } \tilde{l} \in\left[0, \frac{\epsilon}{2}\right], \tilde{r} \in\left[\frac{1-\epsilon}{2}, \frac{1}{2}\right], \tilde{l}+\tilde{r}=\frac{1}{2}\right\}, \\
\operatorname{Bay}\left(\Phi_{(s, t)}^{0} \mid F_{1,2}^{1}\right) & =\{(0,0,0,0,0,1,0)\}, \\
\operatorname{Bay}\left(\Phi_{(s, t)}^{0} \mid F_{1,3}^{1}\right) & =\{(0,0,0,0,0,0,1)\} .
\end{aligned}
$$

The interim utility at $F_{1}^{1}$ is

$$
\begin{aligned}
U^{i}((1-m, m)) & =\min _{(\tilde{l}, \tilde{r})} 101 \tilde{l}(1-m)+101 \tilde{r} m+100 \tilde{r}(1-m)(s+t) \\
& =\min _{(\tilde{l}, \tilde{r})} \tilde{l} \underbrace{101(1-m)}_{>0}+\tilde{r} \underbrace{(100+m)}_{>0} .
\end{aligned}
$$

The worst case belief depends on $m$. If $101(1-m)>100+m$ the worst case belief is such that $\tilde{l}=0$ and $\tilde{r}=\frac{1}{2}$. If $101(1-m) \leq 100+m$ the worst case belief is $\tilde{l}=\frac{\epsilon}{2}$ and $\tilde{r}=\frac{1-\epsilon}{2}$. Since $101(1-m)>100+m \Leftrightarrow m<\frac{1}{102}$, player 1 maximize the following interim utility

$$
\begin{aligned}
U^{i}((1-m, m)) & =\left\{\begin{array}{lr}
\frac{1}{2}(100+m) & \text { if } m<\frac{1}{102} \\
101(1-m) \frac{\epsilon}{2}+\frac{1-\epsilon}{2}(100+m) & \text { if } m \geq \frac{1}{102}
\end{array}\right. \\
& = \begin{cases}\frac{1}{2}(100+m) & \text { if } m<\frac{1}{102} \\
m \frac{1-102 \epsilon}{2}+\frac{101 \epsilon+100(1-\epsilon)}{2} & \text { if } m \geq \frac{1}{102} .\end{cases}
\end{aligned}
$$

This shows, as long as $\epsilon>\frac{1}{102}$ the optimal strategy at the interim stage is $m^{*}=\frac{1}{102}<1$. Hence, player 1 changes his optimal strategy when he observes the information set $F_{1}^{1}$. This proves dynamically inconsistent behavior.

\section{B.1.2 Dynamic Consistency with updating rule of Hanany and Klibanoff (2007)}

Here we show that the updating rules characterized in Hanany and Klibanoff (2007) lead to a different dynamically consistent equilibrium as stability under pasting. To rule out the case without inconsistency we assume that $\epsilon>\frac{1}{102}$. We focus on player 1 and show that the ambiguity maximizing updating rule as defined in Hanany and Klibanoff (2007) implies that the optimal interim strategy of player 1 is playing $m$ with probability 1 .

Following the notation of Hanany and Klibanoff (2007), the updated event is $E=$ $\{L, R\}$ and the optimal ex ante choice is $m=1$. Then the set of measures supporting the conditional optimality of $m=1$ are ${ }^{14}$

$$
Q^{E, m=1, \Sigma_{1}}=\left\{(l, r, o) \in \mathcal{P} \text { s.t. } l<\frac{1}{102}\right\} .
$$

\footnotetext{
${ }^{14} l<\frac{1}{102}$ follows from similar calculation as the interim maximization. If $l>\frac{1}{102}$ the ex ante worst case belief would give a positive probability to $l$ that is high enough to change the optimal strategy of player 1 and playing $m^{*}<1$ would be optimal. With $l<\frac{1}{102}$ playing $m^{*}=1$ is always optimal.
} 
We will show that updating only $Q^{E, m=1, \Sigma_{1}}$ implies that $m=1$ is optimal at the interim stage. The Bayesian update given $E$ of $Q^{E, m=1, \Sigma_{1}}$ is

$$
\begin{aligned}
Q_{E}^{E, m=1, \Sigma_{1}} & =\left\{(\tilde{l}, \tilde{r}, 0) \text { s.t. } \tilde{l}=\frac{l}{l+r}=1-\tilde{r},(l, r, o) \in Q^{E, m=1, \Sigma_{1}}\right\} \\
& =\left\{(\tilde{l}, \tilde{r}, 0) \text { s.t. } \tilde{l}=1-\tilde{r}, \tilde{l} \in\left[0, \frac{1}{102}\right), \tilde{r} \in[1-\epsilon, 1]\right\}
\end{aligned}
$$

Maximizing the interim worst case expected utility given $Q_{E}^{E, m=1, \Sigma_{1}}$ yields

$$
\begin{aligned}
& \max _{m \in[0,1]} \min _{(\tilde{l}, \tilde{r}) \in Q_{E}^{E, m=1, \Sigma_{1}}} \tilde{l}(1-m) 101+(1-\tilde{l}) 101+(1-\tilde{l})(1-m) 100 \\
& =\max _{m \in[0,1] \tilde{l} \in\left[0, \frac{1}{102}\right)} \min _{l} \tilde{l}(1-102 m)+100+m \\
& \Rightarrow \text { worst case belief } \tilde{l}^{*}= \begin{cases}0 & \text { if } m<\frac{1}{102} \\
\frac{1}{102}-\delta & \text { if } m \geq \frac{1}{102}\end{cases}
\end{aligned}
$$

where $\delta>0 .{ }^{15}$ Then player 1 maximize

$$
\max _{m \in[0,1]} \begin{cases}100+m & \text { if } m<\frac{1}{102} \\ \frac{1}{102}+100+\left(1-102\left(\frac{1}{102}-\delta\right)\right) m & \text { if } m \geq \frac{1}{102}\end{cases}
$$

Since $\left(1-102\left(\frac{1}{102}-\delta\right)\right)$ is strictly greater 0 player 1 chooses $m^{*}=1$ at the interim stage. Hence, using the updating rules of Hanany and Klibanoff (2007) leads to a dynamically consistent equilibrium in which player 1 chooses $m^{*}=1$ at the interim and ex-ante stage.

This shows the difference between the approach of Hanany and Klibanoff (2007) and stability under pasting. The updating rules of Hanany and Klibanoff (2007) give an higher weight to the ex-ante optimal choice. Therefore the dynamically consistent strategy equals the ex-ante optimal strategy. In our setting players adjust their ex-ante beliefs. Therefore, using our approach leads to an equilibrium in which player 1 chooses the interim optimal strategy at the ex-ante stage.

\section{B.2 Greenbergs Peace Negotiation Example}

Denote the history that country A will be punished, country A plays peace and country B war by Apw. The other histories are denoted similarly.

First we construct the belief sets $\Phi_{\sigma_{-i}}^{0}$ and show that they are stable under pasting. Then we show that for the game with restricted strategy set $\Sigma^{k}$ there exist an interim, an ex-ante and sequential equilibrium such that no player chooses war. Remember that $\Sigma^{k}$ restrict the strategy of country $A$ and $B$ such that $\alpha \in\left[\epsilon_{A}^{k}, 1-\epsilon_{A}^{k}\right]$ and $\beta \in\left[\epsilon_{B}^{k}, 1-\epsilon_{B}^{k}\right]$ with $\epsilon_{i}^{k}<\frac{1}{2}$

\footnotetext{
${ }^{15}$ Since $\tilde{l} \in\left[0, \frac{1}{102}\right)$ it has to strictly smaller than $\frac{1}{102}$.
} 
and $\epsilon_{i}^{k} \rightarrow \infty$ if $k \rightarrow \infty$.

- Belief sets and stability under pasting:

The information filtration $\mathcal{F}_{i}^{t}$ and the prior beliefs $\Phi_{\sigma_{-i}}^{0}$ are given by

$$
\begin{aligned}
& \mathcal{F}_{i}^{0}=\{A w, A p w, A p p, B p p, B p w, B w\}=H, \\
& \mathcal{F}_{A}^{1}=\mathcal{F}_{i}^{0}, \\
& \mathcal{F}_{B}^{1}=\{\{A w, B w\},\{A p w, A p p, B p p, B p w\}\}
\end{aligned}
$$

and

$$
\begin{array}{r}
\Phi_{\sigma_{-A}}^{0}=\{(\mu, \mu \beta, \mu(1-\beta),(1-\mu)(1-\beta),(1-\mu) \beta,(1-\mu)) \text { s.t. } \mu \in[\underline{\mu}, \bar{\mu}]\}, \\
\Phi_{\sigma_{-B}}^{0}=\{(\mu \alpha, \mu(1-\alpha), \mu(1-\alpha),(1-\mu)(1-\alpha),(1-\mu)(1-\alpha),(1-\mu) \alpha) \\
\text { s.t. } \mu \in[\underline{\mu}, \bar{\mu}]\} .
\end{array}
$$

We denote with $F_{B, 1}^{2}$ and $F_{B, 2}^{2}$ the first and second element of $\mathcal{F}_{B}^{1}$. It is easy to verify that $\Phi_{\sigma_{-i}}^{0}$ is stable under pasting:

The marginal belief of player 1 is $\phi\left(F_{A}^{1}\right)=2$. Updating $\Phi_{\sigma_{-A}}^{0}$ prior by prior leads to

$$
\Phi_{\sigma_{-A}}^{1}=\left\{\left(\frac{\mu}{2}, \frac{\mu \beta}{2}, \frac{\mu(1-\beta)}{2}, \frac{(1-\mu)(1-\beta)}{2}, \frac{(1-\mu) \beta}{2}, \frac{1-\mu}{2}\right) \text { s.t. } \mu \in[\underline{\mu}, \bar{\mu}]\right\} \text {. }
$$

Then the pasting of marginal an updated beliefs shows $\operatorname{rect}\left(\Phi_{\sigma_{-A}}^{0}\right)=\Phi_{\sigma_{-A}}^{0}$.

For player $B$ we have to differ between the two information sets $F_{B, 1}^{1}$ and $F_{B, 2}^{1}$. Marginals and updated beliefs are

$$
\begin{gathered}
\phi\left(F_{B, 1}^{1}\right)=\alpha, \\
\phi\left(F_{B, 2}^{1}\right)=2(1-\alpha), \\
\Phi_{\sigma_{-B}}^{1}\left(F_{B, 1}^{1}\right)=\{(\mu, 0,0,0,0,1-\mu) \text { s.t. } \mu \in[[\mu], \bar{\mu}]\}, \\
\Phi_{\sigma_{-B}}^{1}\left(F_{B, 2}^{2}\right)=\left\{\left(0, \frac{\mu}{2}, \frac{\mu}{2}, \frac{(1-\mu)}{2}, \frac{(1-\mu)}{2}, 0\right) \text { s.t. } \mu \in[\underline{\mu}, \bar{\mu}]\right\} .
\end{gathered}
$$

The pasting of marginal and updated beliefs leads to

$$
\begin{aligned}
& \operatorname{rect}\left(\Phi_{\sigma_{-B}}^{0}\right) \\
& =\{(\mu \alpha, \mu(1-\alpha), \mu(1-\alpha),(1-\mu)(1-\alpha),(1-\mu)(1-\alpha),(1-\mu) \alpha) \text { s.t. } \mu \in[\underline{\mu}, \bar{\mu}]\} \\
& =\Phi_{\sigma_{-B}}^{0} .
\end{aligned}
$$


Hence, $\Phi_{\sigma_{-A}}^{0}$ and $\Phi_{\sigma_{-B}}^{0}$ are stable under pasting.

- interim equilibrium

We first calculate the best response of country B at the interim stage.

$$
\begin{aligned}
& \max _{\beta \in\left[\epsilon_{B}^{k}, 1-\epsilon_{B}^{k}\right]} \min _{\phi \in \Phi_{\sigma_{-B}^{1}}^{1}\left(F_{B, 2}^{1}\right)} 9 \beta \phi(A p w)+4(1-\beta) \phi(A p p)+4(1-\beta) \phi(B p p)+0 \beta \phi(B p w) \\
& =\max _{\beta \in\left[\epsilon_{B}^{k}, 1-\epsilon_{B}^{k}\right] \mu \in[\underline{\mu}, \bar{\mu}]} 9 \beta \frac{\mu}{2}+4(1-\beta) \frac{\mu}{2}+4(1-\beta) \frac{1-\mu}{2} \\
& =\max _{\beta \in\left[\epsilon_{B}^{k}, 1-\epsilon_{B}^{k}\right] \mu \in[\underline{\mu}, \bar{\mu}]} \min _{2} \beta \mu+2(1-\beta) \\
& =\max _{\beta \in\left[\epsilon_{B}^{k}, 1-\epsilon_{B}^{k}\right]} \frac{9}{2} \underline{\mu} \beta+2(1-\beta) \\
& \Rightarrow \beta^{*}= \begin{cases}1-\epsilon_{B}^{k} & \text { if } 9 \underline{\mu}>4 \\
{\left[\epsilon_{B}^{k}, 1-\epsilon_{B}^{k}\right]} & \text { if } 9 \underline{\mu}=4 \\
\epsilon_{B}^{k} & \text { if } 9 \underline{\mu}<4 .\end{cases}
\end{aligned}
$$

Country A solve the following maximization problem at the interim stage

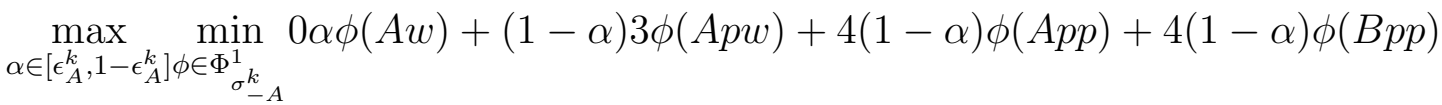

$$
\begin{aligned}
& +6(1-\alpha) \phi(B p w)+9 \alpha \phi(B w)
\end{aligned}
$$

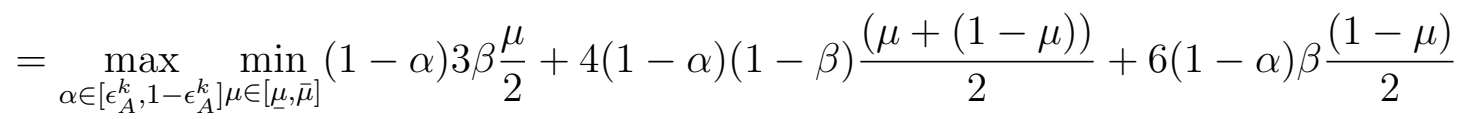

$$
\begin{aligned}
& +9 \alpha \frac{(1-\mu)}{2} \\
& =\frac{1}{2} \max _{\alpha \in\left[\epsilon_{A}^{k}, 1-\epsilon_{A}^{k}\right] \mu \in[\underline{\mu}, \bar{\mu}]} 3(1-\alpha) \beta(\mu+(1-\mu)+4(1-\alpha)(1-\beta)+3(1-\alpha) \beta(1-\mu) \\
& +9 \alpha(1-\mu) \\
& =\frac{1}{2} \max _{\alpha \in\left[\epsilon_{A}^{k}, 1-\epsilon_{A}^{k}\right] \mu \in[\underline{\mu}, \bar{\mu}]} 3(1-\alpha) \beta+4(1-\alpha)(1-\beta)+(1-\mu) \underbrace{(3(1-\alpha) \beta+9 \alpha)}_{>0} \\
& =\frac{1}{2} \max _{\alpha \in\left[\epsilon_{A}^{k}, 1-\epsilon_{A}^{k}\right]} 3(1-\alpha) \beta+4(1-\alpha)(1-\beta)+(1-\bar{\mu})(3(1-\alpha) \beta+9 \alpha) \\
& =\frac{1}{2} \max _{\alpha \in\left[\epsilon_{A}^{k}, 1-\epsilon_{A}^{k}\right]} 3(1-\alpha)+(1-\alpha)(1-\beta)+3(1-\alpha) \beta(1-\bar{\mu})+9 \alpha(1-\bar{\mu})
\end{aligned}
$$




$$
\begin{aligned}
& =\frac{1}{2} \max _{\alpha \in\left[\epsilon_{A}^{k}, 1-\epsilon_{A}^{k}\right]} \alpha(-3-(1-\beta)-3 \beta(1-\bar{\mu})+9(1-\bar{\mu}))+3+(1-\beta)+3(1-\bar{\mu}) \beta \\
& =\frac{1}{2} \max _{\alpha \in\left[\epsilon_{A}^{k}, 1-\epsilon_{A}^{k}\right]} \alpha(5-2 \beta+3 \bar{\mu}(\beta-3))+3+(1-\beta)+3(1-\bar{\mu}) \beta
\end{aligned}
$$

$$
\Rightarrow \alpha^{*}= \begin{cases}\epsilon_{A}^{k} & \text { if } 5-2 \beta+3 \bar{\mu}(\beta-3)<0 \\ {\left[\epsilon_{A}^{k}, 1-\epsilon_{A}^{k}\right]} & \text { if } 5-2 \beta+3 \bar{\mu}(\beta-3)=0 \\ 1-\epsilon_{A}^{k} & \text { if } 5-2 \beta+3 \bar{\mu}(\beta-3)>0 .\end{cases}
$$

Now we show that $\alpha^{*}=\epsilon_{A}^{k}, \beta^{*}=\epsilon_{B}^{k}$ and $\Psi^{1}=\left(\Phi_{\sigma_{-A}}^{1}, \Phi_{\sigma_{-B}}^{1}\right)$ form an equilibrium.

$$
\begin{gathered}
\beta^{*}=\epsilon_{B}^{k} \Leftrightarrow \underline{\mu}<\frac{4}{9} \\
\Rightarrow 5-2 \beta+3 \bar{\mu}(\beta-3)=5-9 \bar{\mu}<0 \Leftrightarrow \bar{\mu}>\frac{5}{9} \\
\Rightarrow \alpha^{*}=\epsilon_{A}^{k} .
\end{gathered}
$$

This is satisfied since $[\underline{\mu}, \bar{\mu}] \supset\left[\frac{4}{9}, \frac{5}{9}\right]$. Hence, there exist an equilibrium in which both players play peace with probability 1 .

- ex-ante equilibrium:

We show that $\alpha^{*}=\epsilon_{A}^{k}, \beta^{*}=\epsilon_{B}^{k}$ are an ex-ante equilibrium as well:

Let us start with player $A$. We show that given $\beta=\epsilon_{B}^{k}$ and $\Phi_{\sigma_{-A}}^{0}$ the optimal strategy of player $A$ is $\alpha^{*}=\epsilon_{A}^{k}$.

$$
\Phi_{\sigma_{-A}}^{0}=\left\{\left(\mu, \mu \epsilon_{B}^{k}, \mu\left(1-\epsilon_{B}^{k}\right),(1-\mu)\left(1-\epsilon_{B}^{k}\right),(1-\mu) \epsilon_{B}^{k},(1-\mu)\right) \text { s.t. } \mu \in[\underline{\mu}, \bar{\mu}]\right\}
$$

and

$$
\begin{aligned}
& \max _{\alpha \in\left[\epsilon_{A}^{k}, 1-\epsilon_{A}^{k}\right]} \min _{\phi \in \Phi_{\sigma_{-A}}^{0}} \phi(A w) \alpha 0+3 \phi(\text { Apw })(1-\alpha)+4 \phi(\text { App })(1-\alpha)+4 \phi(B p p)(1-\alpha) \\
& +6 \phi(B p w)(1-\alpha)+9 \phi(B w) \alpha \\
& =\max _{\alpha} \min _{\mu \in[\mu, \bar{\mu}]} 3 \mu(1-\alpha) \epsilon_{B}^{k}+4 \mu(1-\alpha)\left(1-\epsilon_{B}^{k}\right)+4(1-\mu)(1-\alpha)\left(2-\epsilon_{B}^{k}\right) \\
& +6(1-\alpha)(1-\mu) \epsilon_{B}^{k}+9(1-\mu) \alpha \\
& =\max _{\alpha} \min _{\mu} 3 \mu(1-\alpha) \epsilon_{B}^{k}+4(1-\alpha)\left(1-\epsilon_{B}^{k}\right)+6(1-\alpha)(1-\mu) \epsilon_{B}^{k}+9(1-\mu) \alpha \\
& =\max _{\alpha} \min _{\mu} \mu\left(3(1-\alpha) \epsilon_{B}^{k}-6(1-\alpha) \epsilon_{B}^{k}-9 \alpha\right)+4(1-\alpha)\left(1-\epsilon_{B}^{k}\right)+6(1-\alpha) \epsilon_{B}^{k}+9 \alpha \\
& =\max _{\alpha} \min _{\mu} \mu \underbrace{\left(-3(1-\alpha) \epsilon_{B}^{k}-9 \alpha\right)}_{<0<\alpha, \epsilon_{B}^{k}}+4(1-\alpha)\left(1-\epsilon_{B}^{k}\right)+6(1-\alpha) \epsilon_{B}^{k}+9 \alpha
\end{aligned}
$$




$$
\begin{aligned}
& =\max _{\alpha} \bar{\mu}\left(-3(1-\alpha) \epsilon_{B}^{k}-9 \alpha\right)+4(1-\alpha)\left(1-\epsilon_{B}^{k}\right)+6(1-\alpha) \epsilon_{B}^{k}+9 \alpha \\
& =\max _{\alpha} \alpha\left(-9 \bar{\mu}+3 \epsilon_{B}^{k} \bar{\mu}-4\left(1-\epsilon_{B}^{k}\right)-6 \epsilon_{B}^{k}+9\right)-3 \epsilon_{B}^{k} \bar{\mu}+4\left(1-\epsilon_{B}^{k}\right)+6 \epsilon_{B}^{k} .
\end{aligned}
$$

This implies that

$$
\begin{aligned}
\alpha^{*}=\epsilon_{A}^{k} & \Leftrightarrow-9 \bar{\mu}+3 \epsilon_{B}^{k} \bar{\mu}-4\left(1-\epsilon_{B}^{k}\right)-6 \epsilon_{B}^{k}+9<0 \\
& \Leftrightarrow \bar{\mu}\left(3 \epsilon_{B}^{k}-9\right)<2 \epsilon_{B}^{k}-5 \\
& \Leftrightarrow \bar{\mu}>\frac{5-2 \epsilon_{B}^{k}}{9-3 \epsilon_{B}^{k}} .
\end{aligned}
$$

which is satisfied for all $\epsilon_{B}^{k}$ since $\bar{\mu}>\frac{5}{9}>\frac{5-2 \epsilon_{B}^{k}}{9-3 \epsilon_{B}^{k}}$ for all $\epsilon_{B}^{k}>0$. Hence, $\alpha^{*}=\epsilon_{A}^{k}$ is optimal.

The ex-ante belief of player $B$ given $\alpha=\epsilon_{A}^{k}$ is

$$
\begin{gathered}
\Phi_{\sigma_{-B}}^{0}=\left\{\left(\mu \epsilon_{A}^{k}, \mu\left(1-\epsilon_{A}^{k}\right), \mu\left(1-\epsilon_{A}^{k}\right),(1-\mu)\left(1-\epsilon_{A}^{k}\right),(1-\mu)\left(1-\epsilon_{A}^{k}\right),(1-\mu) \epsilon_{A}^{k}\right)\right. \\
\text { s.t. } \mu \in[\underline{\mu}, \bar{\mu}]\}
\end{gathered}
$$

and

$$
\begin{aligned}
\max _{\beta \in\left[\epsilon_{B}^{k}, 1-\epsilon_{B}^{k}\right]} & \min _{\phi \in \Phi_{\sigma-B}^{0}} 9 \phi(A w)+9 \beta \phi(A p w)+4(1-\beta) \phi(A p p)+4(1-\beta) \phi(B p p)+0+0 \\
& =\max _{\beta} \min _{\mu \in[\underline{\mu}, \bar{\mu}]} 9 \mu \epsilon_{A}^{k}+9 \beta \mu\left(1-\epsilon_{A}^{k}\right)+4(1-\beta) \mu+4(1-\beta)\left(1-\epsilon_{A}^{k}\right) \\
& =\max _{\beta} \min _{\mu} \mu \underbrace{\left(9 \epsilon_{A}^{k}+9 \beta\left(1-\epsilon_{A}^{k}\right)\right)}_{>0}+4\left(1-\epsilon_{A}^{k}\right)(1-\beta) \\
& =\max _{\beta} \underline{\mu}\left(9 \epsilon_{A}^{k}\right. \\
& =\max _{\beta} \beta\left(9 \underline{\mu}\left(1-\epsilon_{A}^{k}\right)\right)+4\left(1-\epsilon_{A}^{k}\right)(1-\beta) \\
& \left.\left.\left.=\max _{\beta} \beta(\underbrace{\left(1-\epsilon_{A}^{k}\right.}_{>0})-4\left(1-\epsilon_{A}^{k}\right)\right)+4\left(1-\epsilon_{A}^{k}\right)+9 \epsilon_{A}^{k} \underline{\mu}-4\right)\right)+4\left(1-\epsilon_{A}^{k}\right)+9 \epsilon_{A}^{k} \underline{\mu} \\
\Rightarrow & \beta^{*}=\epsilon_{B}^{k} \Leftrightarrow \underline{\mu}<\frac{4}{9}
\end{aligned}
$$

which is satisfied by the assumptions on $\mathcal{P}$. Hence, $\beta^{*}=\epsilon_{B}^{k}$ is optimal and $\left(\alpha^{*}, \beta^{*}\right)=$ $\left(\epsilon_{A}^{k}, \epsilon_{B}^{k}\right)$ is an ex-ante equilibrium with rectangular beliefs for the game with restricted strategy set $\Gamma^{k}$.

- sequential equilibrium:

$\left(\alpha^{*}, \beta^{*}\right)=\left(\epsilon_{A}^{k}, \epsilon_{B}^{k}\right)$ converge to $\left(\alpha^{*}, \beta^{*}\right)=(0,0)$ for $k \rightarrow \infty$ and $\left(\alpha^{*}, \beta^{*}\right)=\left(\epsilon_{A}^{k}, \epsilon_{B}^{k}\right)$ together with the belief specified above form an ex-ante and interim equilibrium 
with rectangular belief. Therefore, $\left(\alpha^{*}, \beta^{*}\right)=(0,0)$ satisfies sequential rationality. Furthermore, $\Phi_{\beta=\epsilon_{B}^{k}}^{0}$ and $\Phi_{\alpha=\epsilon_{A}^{k}}^{0}$ converge to $\Phi_{\beta=0}^{0}$ and $\Phi_{\alpha=0}^{0}$ for $k \rightarrow \infty$, respectively. Thus the belief system specified above is consistent w.r.t. $\left(\alpha^{*}, \beta^{*}\right)=(0,0)$. This proves that $\left(\left(\alpha^{*}, \beta^{*}\right)=(0,0),\left(\Phi_{\beta=0}^{0}, \Phi_{\alpha=0}^{0}\right)\right)$ forms a sequential equilibrium.

\section{Equilibria without ambiguity}

In this part we show that without ambiguity there exist no equilibrium in which country A and B play peace.

case 1: $\beta^{*}=0$

$$
\begin{aligned}
& \Rightarrow \mu<\frac{4}{9} \\
& \Rightarrow 5-2 \beta+3 \overline{\tilde{\mu}}_{A}(\beta-3)=5-9 \mu>5-9 \cdot \frac{4}{9}=1>0 \\
& \Rightarrow \alpha^{*}=1
\end{aligned}
$$

Eq1: $\beta^{*}=0, \alpha^{*}=1, \mu<\frac{4}{9} \Rightarrow$ war

case $2: \beta=1$

$$
\begin{aligned}
& \Rightarrow \mu>\frac{4}{9} \\
& \Rightarrow 5-2 \beta+3 \overline{\tilde{\mu}}_{A}(\beta-3)=3-6 \mu<0 \Leftrightarrow \mu>\frac{1}{2}
\end{aligned}
$$

$\mathrm{Eq} 2: \beta^{*}=1, \alpha^{*}=0, \mu>\frac{1}{2} \Rightarrow$ war

Eq3: $\beta^{*}=1, \alpha^{*}=1, \mu<\frac{1}{2} \Rightarrow$ war

Eq4: $\beta^{*}=1, \alpha^{*} \in[0,1], \mu=\frac{1}{2} \Rightarrow$ war

case 3: $\beta^{*} \in[0,1]$

$$
\begin{aligned}
& \Rightarrow \mu=\frac{4}{9} \\
& \Rightarrow 5-2 \beta+3 \overline{\tilde{\mu}}_{A}(\beta-3)=1-\frac{2}{3} \beta>0 \\
& \Rightarrow \alpha^{*}=1
\end{aligned}
$$

Eq5: $\beta^{*} \in[0,1], \alpha^{*}=1, \mu=\frac{4}{9} \Rightarrow$ war

The calculations above show that there is no equilibrium in which both players play peace. 


\section{B.3 Example: Rectangular Belief Sets Depend on Strategies of Opponents}

Example 3 The following example shows that the rectangular hull of a prior belief set depends on the strategy of the opponents. We focus on player 2 and show that his rectangular prior belief set differs if player 1 plays $A$ or $B$. The game is depicted in Figure 4. Nature chooses the state $L, R$ or $O$. Player 1 moves first. He cannot observe the state and can choose between $A$ and $B$. If the state is $L$ and he plays $B$ the game ends. If the state is $O$ and he plays $A$ the game ends as well. Player 2 observes the action played by player 1 and whether the game continue or not. Since we focus on player 2, we only specify the payoffs of player 2 . The imprecise probabilistic information is the same as in our running example

$$
\mathcal{P}=\{(1-\epsilon)(0,1,0)+\epsilon(l, r, o):(l, r, o) \in \Delta\}
$$

where $l, r$, and o denote the probability of $L, R$ and $O$, respectively, and $\epsilon>0$ is some fixed constant. Furthermore, let $a$ and $b$ denote the probabilities that player 1 plays $A$ or

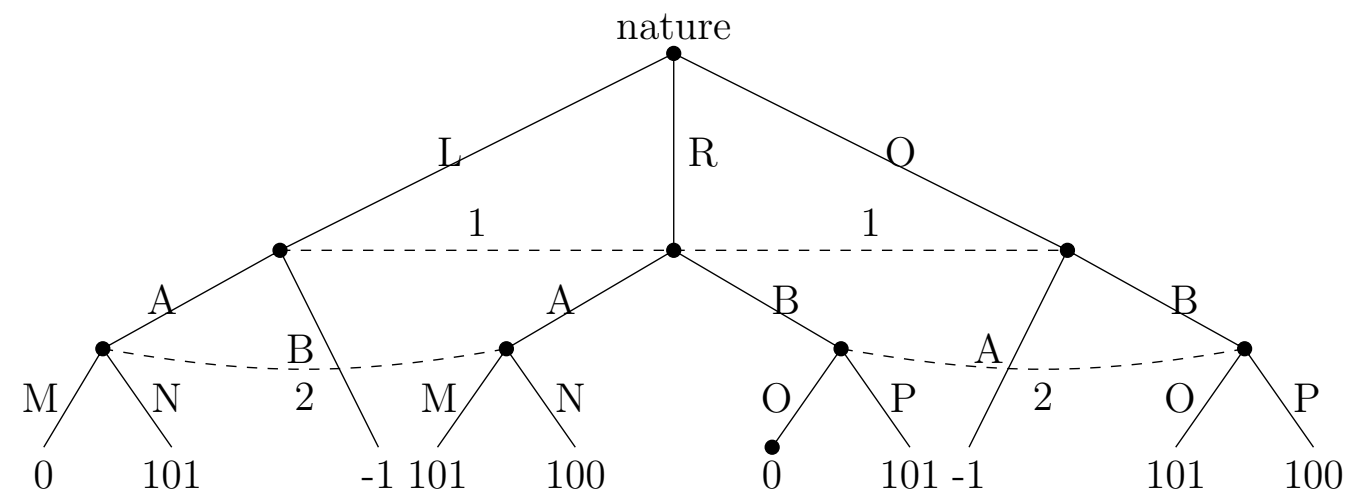

Figure 4: Example 3

$B$, respectively, and similar $m$ and $n$ the probabilities that player 2 plays $M$ or $N$.

The set of histories $H$, the information partition $\mathcal{F}_{2}^{1}$ and the prior belief set $\Phi_{\sigma_{-2}}^{0}$ of player 2 are

$$
\begin{aligned}
H & =\{L A N, L A M, L B, R A N, R A M, R B O, R B P, O A, O B O, O B P\}, \\
\mathcal{F}_{2}^{1} & =\{L A N, L A M, R A N, R A M\},\{R B O, R B P, O B O, O B P\},\{L B\}\{O A\}\}, \\
\Phi_{\sigma_{-2}}^{0} & =\{(l a, l a, l b, r a, r a, r b, r b, o a, o b, o b) \text { with }(l, r, o) \in \mathcal{P}\} .
\end{aligned}
$$

Moreover, let $F_{2, k}^{1}$ with $k=1,2,3,4$ denote the elements of the partition $\mathcal{F}_{2}^{1}$ in the same order as they are denoted above. We first look at the case where player 1 plays A with probability 1. Then we compare it with the case where player 1 plays $B$ with probability 1. Please notice that if the probability of $A$ is 1 or 0 the game is very similar to our running example. 
- player 1 plays $A$

The prior belief set and marginal beliefs are given by

$$
\begin{aligned}
\Phi_{A}^{0} & =\{(l, l, 0, r, r, 0,0, o, 0,0) \text { s.t. }(l, r, o) \in \mathcal{P}\} \\
\phi\left(F_{2,1}^{1}\right) & =2 l+2 r \\
\phi\left(F_{2,2}^{1}\right) & =0 \\
\phi\left(F_{2,3}^{1}\right) & =0 \\
\phi\left(F_{2,4}^{1}\right) & =o=1-l-r .
\end{aligned}
$$

For information sets with positive marginal probability Bayes' rule is well defined and the Bayesian updates are given by

$$
\begin{aligned}
\operatorname{Bay}\left(\Phi_{A}^{0} \mid F_{2,1}^{1}\right) & =\left\{\left(\frac{l}{2(l+r)}, \frac{l}{2(l+r)}, 0, \frac{r}{2(l+r)}, \frac{r}{2(l+r)}, 0,0,0,0,0\right)\right. \\
\text { s.t. } l \in[0, \epsilon], r \in[1-\epsilon, 1]\} & \\
& =\left\{(\tilde{l}, \tilde{l}, 0, \tilde{r}, \tilde{r}, 0,0,0,0,0) \text { s.t. } \tilde{l} \in\left[0, \frac{\epsilon}{2}\right], \tilde{r}=\frac{1}{2}-\tilde{l}\right\}, \\
\operatorname{Bay}\left(\Phi_{A}^{0} \mid F_{2,4}^{1}\right) & =(0,0,0,0,0,0,0,1,0,0) .
\end{aligned}
$$

The rectangular hull $\operatorname{rect}\left(\Phi_{A}^{0}\right)$ giving player 1 play $A$ is

$$
\begin{aligned}
\operatorname{rect}\left(\Phi_{A}^{0}\right)= & \operatorname{conv}\{(\epsilon, \epsilon, 0,(1-\epsilon),(1-\epsilon), 0,0,0,0,0),(0,0,0,(1-\epsilon),(1-\epsilon), 0,0, \epsilon, 0,0) \\
& \left.(0,0,0,1,1,0,0,0,0,0),\left(\epsilon(1-\epsilon), \epsilon(1-\epsilon), 0,(1-\epsilon)^{2},(1-\epsilon)^{2}, 0,0, \epsilon, 0,0\right)\right\} .
\end{aligned}
$$

- player 1 plays $B$

The prior belief set and the marginal beliefs are given by

$$
\begin{aligned}
\Phi_{B}^{0} & =\{(0,0, l, 0,0, r, r, 0, o, o) \text { s.t. }(l, r, o) \in \mathcal{P}\}, \\
\phi\left(F_{2,1}^{1}\right) & =0 \\
\phi\left(F_{2,2}^{1}\right) & =2 o+2 r \\
\phi\left(F_{2,3}^{1}\right) & =l \\
\phi\left(F_{2,4}^{1}\right) & =0 .
\end{aligned}
$$

The Bayesian updates for information sets with positive marginal probability are

$$
\begin{gathered}
\operatorname{Bay}\left(\Phi_{B}^{0} \mid F_{2,2}^{1}\right)=\left\{\left(0,0,0,0,0, \frac{r}{2(o+r)}, \frac{r}{2(o+r)}, 0, \frac{o}{2(o+r)}, \frac{o}{2(o+r)}\right)\right. \\
\text { s.t. } o \in[0, \epsilon], r \in[1-\epsilon, 1]\}
\end{gathered}
$$




$$
\begin{aligned}
& =\left\{(0,0,0,0,0, \tilde{r}, \tilde{r}, 0, \tilde{o}, \tilde{o}) \text { s.t. } \tilde{o} \in\left[0, \frac{\epsilon}{2}\right], \tilde{r}=1-\tilde{l}\right\}, \\
\operatorname{Bay}\left(\Phi_{A}^{0} \mid F_{2,3}^{1}\right) & =(0,0,1,0,0,0,0,0,0,0) .
\end{aligned}
$$

The rectangular hull $\operatorname{rect}\left(\Phi_{B}^{0}\right)$ giving player 1 plays $B$ is

$$
\begin{array}{r}
\operatorname{rect}\left(\Phi_{B}^{0}\right)=\operatorname{conv}\{(0,0,0,0,0,1-\epsilon, 1-\epsilon, 0, \epsilon, \epsilon),(0,0, \epsilon, 0,0,(1-\epsilon),(1-\epsilon), 0,0,0) \\
\left.(0,0,0,0,0,1,1,0,0,0),\left(0,0, \epsilon, 0,0,(1-\epsilon)^{2},(1-\epsilon)^{2}, 0, \epsilon(1-\epsilon), \epsilon(1-\epsilon)\right)\right\}
\end{array}
$$

The above calculations show that $\operatorname{rect}\left(\Phi_{A}^{0}\right)$ and $\operatorname{rect}\left(\Phi_{B}^{0}\right)$ not only differ in the histories with zero probability but also in the possible values for $l$ and o. For $\operatorname{rect}\left(\Phi_{A}^{0}\right)$ the probability $l$ takes values in the interval $[0,(1-\epsilon) \epsilon]$, for $\operatorname{rect}\left(\Phi_{B}^{0}\right)$ the probability $l$ takes only values in the interval $[0, \epsilon]$ and vice versa for o. Therefore, the strategy of player 1 essentially influence the rectangular hull of the prior belief set.

\section{Dynamic Consistency with 2 types or states}

In games with only two possible types or states, i.e. $\left|H^{0}\right|=2$, ambiguity has no influence on dynamic consistency. Let us assume that $\left|H^{0}\right|=2$. Then the imprecise probabilistic information has the following form

$$
\mathcal{P}=\{(\pi, 1-\pi) \text { s.t. } \pi \in[\underline{\pi}, \bar{\pi}]\} .
$$

There are only two cases that can occur at an interim stage. Either a player learns which type is the true type or he does not learn the true type. In signaling games the first case corresponds to separating equilibria and the second to pooling equilibria. Let us look at both cases separately.

- First assume that a player learns the true type at the interim stage. This implies, that the updated probability of a type is either 0 or 1 . Remember the definition of beliefs in our setting, $\phi(h)=p_{\sigma_{-i}}\left(h \mid h^{0}\right) \pi\left(h^{0}\right)$ and let $F_{i}^{t}$ be the information set in which the player learns that the true type is $\bar{h}$. Since player $i$ learns the true type, it follows that for all $h \in F_{i}^{t}$

$$
h^{0}=\bar{h}^{0} .
$$

This implies that $\pi\left(h^{0}\right)=\pi\left(\bar{h}^{0}\right)$ for all $h \in F_{i}^{t}$ with $\phi(h)>0$. Then the marginal belief can be written as

$$
\phi\left(F_{i}^{t}\right)=\sum_{h \in F_{i}^{t}} p_{\sigma_{-i}}\left(h \mid h^{0}\right) \pi\left(h^{0}\right)=\pi\left(\bar{h}^{0}\right) \sum_{h \in F_{i}^{t}} p_{\sigma_{-i}}\left(h \mid h^{0}\right) .
$$


Furthermore, the updated belief is

$$
\begin{aligned}
\tilde{\phi}(h) & =\left\{\begin{array}{lr}
\frac{\pi\left(\bar{h}^{0}\right) p_{\sigma_{-i}}\left(h \mid h^{0}\right)}{\pi\left(\bar{h}^{0}\right) \sum_{h \in F_{i}^{t}} p_{\sigma_{-i}}\left(h \mid h^{0}\right)} & \text { if } h \in F_{i}^{t} \\
0 & \text { otherwise }
\end{array}\right. \\
& = \begin{cases}\frac{p_{\sigma_{-i}}\left(h \mid h^{0}\right)}{\sum_{h \in F_{i}^{t}} p_{\sigma_{-i}}\left(h \mid h^{0}\right)} & \text { if } h \in F_{i}^{t} \\
0 & \text { otherwise. }\end{cases}
\end{aligned}
$$

Now the updated belief is independent of $\pi$. Therefore, pasting any marginal belief with an updated belief would lead to the same prior belief as used for the marginal belief. This means that any belief set $\Phi_{\sigma_{-i}}^{0}$ is stable under pasting.

- Player $i$ does not learn the true type if the strategy of the opponents $\sigma_{-i}$ is completely mixed. If this is the case, Bayes' rule is always well defined. Since there are only 2 types $\mathcal{P}$ has the form noted above. Let $\pi$ denote the probability of type $\bar{h}^{0}$. The updated belief of a history containing type $\overline{h^{0}}$ is then

$$
\tilde{\phi}(h)=\frac{p_{\sigma_{-i}}\left(h \mid \bar{h}^{0}\right) \pi}{\sum_{\substack{h \in h \\ \text { s.t. } h^{0}=\bar{h}^{0}}} p_{\sigma_{-i}}\left(h \mid \bar{h}^{0}\right) \pi+\sum_{\substack{h \in h \\ \text { s.t. } h^{0} \neq \bar{h}^{0}}} p_{\sigma_{-i}}\left(h \mid h^{0}\right)(1-\pi)} .
$$

The derivative of the updated belief w.r.t. $\pi$ is

$$
\frac{\partial \tilde{\phi}}{\partial \pi}=\frac{p_{\sigma_{-i}}\left(h \mid \bar{h}^{0}\right) \sum_{\substack{h \in h \\ \text { s.t. } h^{0} \neq \bar{h}^{0}}} p_{\sigma_{-i}}\left(h \mid h^{0}\right)}{\left(\sum_{\substack{h \in h \\ \text { s.t. } h^{0}=\bar{h}^{0}}} p_{\sigma_{-i}}\left(h \mid \bar{h}^{0}\right) \pi+\sum_{\substack{h \in h \\ \text { s.t. } h^{0} \neq \bar{h}^{0}}} p_{\sigma_{-i}}\left(h \mid h^{0}\right)(1-\pi)\right)^{2}}>0 .
$$

The updated belief is monotone in $\pi$. Therefore, the worst case ex-ante and worst case interim belief would arise from the same $\pi$. This implies dynamic consistency

Both cases lead to dynamically consistent behavior. Hence, in a game with only two states or types dynamic inconsistency never occur.

\section{Further Results}

Additional to Theorem 3 we can show that an equilibrium at stage $t$ implies an equilibrium at stage $t-1$ if a no-profitable one stage deviation property is satisfied.

Definition 13 A tuple $\left(\sigma^{*}, \Psi\right)$ of an strategy profile and an belief system satisfies the noprofitable one stage deviation property at stage $t$ if for all $F_{i}^{t}$

$$
U_{i}^{i}\left(\sigma^{*}, \Psi^{t}\left(F_{i}^{t}\right)\right) \geq U_{i}^{i}\left(\left(\sigma_{i}^{\prime}, \sigma_{-i}^{*}\right), \Psi^{t}\left(F_{i}^{t}\right)\right)
$$

for all $\sigma_{i}^{\prime}$ such that $\sigma_{i}^{\prime}$ equals $\sigma_{i}^{*}$ everywhere except at $F_{i}^{t}$. 
Theorem 5 (interim implies ex-ante equilibria) Assume that $\Psi$ is a belief system which is stable under pasting and that $\left(\sigma^{*}, \Psi^{s}\right)$ satisfies the no-profitable one stage deviation property for all $s<t$. If $\left(\sigma^{*}, \Psi^{t}\right)$ is an interim equilibrium with rectangular beliefs at stage $t$, then $\sigma^{*}$ is an ex-ante equilibrium with rectangular beliefs.

Similarly to Theorem 3 we prove Theorem 5 by showing that due to stability under pasting an interim equilibrium at stage $t$ implies an interim equilibrium at stage $t-1$. Then the recursive structure implies the following Corollary.

Corollary 2 Assume that $\Psi$ is a belief system which is stable under pasting and that $\left(\sigma^{*}, \Psi^{s}\right)$ satisfies the no-profitable one stage deviation property for all $s<t$. If $\left(\sigma^{*}, \Psi^{t}\right)$ is an interim equilibrium with rectangular beliefs at stage $t$, then $\left(\sigma^{*}, \Psi^{t-1}\right)$ is an interim equilibrium with rectangular beliefs at stage $t-1$.

Furthermore Theorem 5 shows that rectangularity and the no-profitable one stage deviation property for all stages implies sequential rationality.

Corollary 3 Let $\Psi$ is a belief system which is stable under pasting and assume that $\left(\sigma^{*}, \Psi^{t}\right)$ satisfies the no-profitable one stage deviation property for all $t \geq 0$. Then $\left(\sigma^{*}, \Psi\right)$ is sequential rational.

Proof. The result follows immediately from Theorem 5 . The no-profitable one stage deviation property of $\left(\sigma^{*}, \Psi^{T}\right)$ at the last stage and stability under pasting imply that $\left(\sigma^{*}, \Psi^{T}\right)$ is an interim equilibrium with rectangular beliefs at the last stage. Then Theorem 5 implies that $\left(\sigma^{*}, \Psi^{t}\right)$ is an interim equilibrium with rectangular beliefs at all stages $t$ and $\sigma^{*}$ is an ex-ante equilibrium with rectangular beliefs.

As in the proof of Theorem 3 the relation of interim and ex ante worst case belief and therefore Lemma 2 and Remark 4 are essentially to prove Theorem 5.

\section{Proof of Theorem 5 (interim implies ex-ante).}

We will prove that an interim equilibrium with rectangular beliefs at stage $t$ implies an interim equilibrium with rectangular beliefs at stage $t-1$. Since this holds for arbitrary $t$, iteration proves the theorem.

Assume that $\left(\sigma^{*}, \Psi^{t}\right)$ is an interim equilibrium with rectangular beliefs at stage $t$. We prove that an arbitrary player $i$ has no incentive to deviate from $\sigma^{*}$ at an arbitrary information set $F_{i}^{t-1}$ if all other players $j \neq i$ play $\sigma_{j}^{*}$.

Fix some arbitrary $F_{i}^{t}$ such that the probability of reaching $F_{i}^{t}$ from $F_{i}^{t-1}$ given $\sigma_{-i}^{*}$ is positive. ${ }^{16}$ Let $\phi^{*, t}$ denote the worst case belief at $F_{i}^{t}$ given $\sigma_{i}^{*}$. Furthermore, let $\bar{\sigma}_{i}$ denote a strategy which is equal to $\sigma_{i}^{*}$ at all stages $s \geq t$ and equal to $\sigma_{i}^{\prime}$ at stage $t-1$. The worst case belief at $F_{i}^{t-1}$ given $\bar{\sigma}_{i}$ is denoted by $\bar{\phi}^{t-1}$. The worst case belief at $F_{i}^{t-1}$ given $\sigma_{i}^{\prime}$ is

\footnotetext{
${ }^{16}$ For information sets with zero probability it follows by the full support assumption on $\mathcal{P}$ that $\phi^{t-1}(h)=$ 0 for all $h \in F_{i}^{t}$ for any $\phi^{t-1} \in \Phi_{i}^{t-1}\left(F_{i}^{t-1}\right)$. Hence, the histories $h \in F_{i}^{t}$ does not influence the expected utility at $F_{i}^{t-1}$.
} 
denoted by $\phi^{\prime, t-1}$.

Since $\sigma_{-i}^{*}$ is fixed the belief sets of player $i$ are fixed as well. Furthermore, the worst case belief at $F_{i}^{t}$ depends only on the part of the strategy of player $i$ which is chosen at stages $s \geq t$. Hence, the worst case beliefs at $F_{i}^{t}$ given $\sigma_{i}^{*}$ and $\bar{\sigma}_{i}$ are the same by the definition of $\bar{\sigma}_{i}$. By Remark 4 we know that the Bayesian update of the worst case belief at $t-1$ is the worst case belief at $t$. This implies

$$
\operatorname{Bay}\left(\bar{\phi}^{t-1}\right)=\phi^{*, t} .
$$

The optimality of $\sigma_{i}^{*}$ at $F_{i}^{t}$ implies

$$
\sum_{h \in F_{i}^{t}} u_{i}(h) p_{\sigma_{i}^{*}}\left(h \mid h^{t}\right) \phi^{*, t} \geq \sum_{h \in F_{i}^{t}} u_{i}(h) p_{\sigma_{i}^{\prime}}\left(h \mid h^{t}\right) \phi^{*, t} .
$$

Combining Equation 8 and Equation 9 implies

$$
\sum_{h \in F_{i}^{t}} u_{i}(h) p_{\sigma_{i}^{*}}\left(h \mid h^{t}\right) \frac{\bar{\phi}^{t-1}(h)}{\sum_{h \in F_{i}^{t}} \bar{\phi}^{t-1}(h)} \geq \sum_{h \in F_{i}^{t}} u_{i}(h) p_{\sigma_{i}^{\prime}}\left(h \mid h^{t}\right) \frac{\bar{\phi}^{t-1}(h)}{\sum_{h \in F_{i}^{t}} \bar{\phi}^{t-1}(h)} .
$$

Now we can cancel the normalization terms of Bayes' rule on both sides and multiply each side with $p_{\sigma_{i}^{\prime}}\left(h^{t} \mid h^{t-1}\right)$. Then, replacing $\bar{\phi}^{t-1}$ with the worst case belief at $t-1$ given $\sigma_{i}^{\prime}$ leads to

$$
\begin{aligned}
\sum_{h \in F_{i}^{t}} u_{i}(h) p_{\sigma_{i}^{*}}\left(h \mid h^{t}\right) p_{\sigma_{i}^{\prime}}\left(h^{t} \mid h^{t-1}\right) \bar{\phi}^{t-1}(h) & \geq \sum_{h \in F_{i}^{t}} u_{i}(h) p_{\sigma_{i}^{\prime}}\left(h \mid h^{t}\right) p_{\sigma_{i}^{\prime}}\left(h^{t} \mid h^{t-1}\right) \bar{\phi}^{t-1}(h) \\
& \geq \sum_{h \in F_{i}^{t}} u_{i}(h) p_{\sigma_{i}^{\prime}}\left(h \mid h^{t}\right) p_{\sigma_{i}^{\prime}}\left(h^{t} \mid h^{t-1}\right) \phi^{\prime, t-1}(h) .
\end{aligned}
$$

This holds for any $F_{i}^{t}$ which is reachable from $F_{i}^{t-1}$. Hence, summation over all this $F_{i}^{t}$ leads to

$$
\sum_{h \in F_{i}^{t-1}} u_{i}(h) p_{\sigma_{i}^{*}}\left(h \mid h^{t}\right) p_{\sigma_{i}^{\prime}}\left(h^{t} \mid h^{t-1}\right) \bar{\phi}^{t-1}(h) \geq \sum_{h \in F_{i}^{t-1}} u_{i}(h) p_{\sigma_{i}^{\prime}}\left(h \mid h^{t}\right) p_{\sigma_{i}^{\prime}}\left(h^{t} \mid h^{t-1}\right) \phi^{\prime, t-1}(h) .
$$

Furthermore, by the no-profitable one stage deviation property it follows that

$$
\sum_{h \in F_{i}^{t-1}} u_{i}(h) p_{\sigma_{i}^{*}}\left(h \mid h^{t-1}\right) \phi^{*, t-1}(h) \geq \sum_{h \in F_{i}^{t-1}} u_{i}(h) p_{\sigma_{i}^{*}}\left(h \mid h^{t}\right) p_{\sigma_{i}^{\prime}}\left(h^{t} \mid h^{t-1}\right) \bar{\phi}^{t-1}(h) .
$$

Combining Equation 10 and Equation 11 leads to

$$
\sum_{h \in F_{i}^{t-1}} u_{i}(h) p_{\sigma_{i}^{*}}\left(h \mid h^{t-1}\right) \phi^{*, t-1} \geq \sum_{h \in F_{i}^{t-1}} u_{i}(h) p_{\sigma_{i}^{\prime}}\left(h \mid h^{t}\right) p_{\sigma_{i}^{\prime}}\left(h^{t} \mid h^{t-1}\right) \phi^{\prime, t-1}(h)
$$


which proves the optimality of $\sigma_{i}^{*}$ at $F_{i}^{t-1}$. This holds for any arbitrary $F_{i}^{t-1}$ and for any arbitrary player $i$. Hence, $\left(\sigma^{*}, \Psi^{t-1}\right)$ is an interim equilibrium with rectangular beliefs at $t-1$. 